\title{
Pentes des Fibrés Vectoriels Adéliques sur un Corps Global.
}

\author{
ÉRIC GAUDRON (*)
}

RÉSUMÉ - Dans les années 90, J.-B. Bost a développé tout un formalisme des pentes des fibrés vectoriels hermitiens sur l'anneau des entiers d'un corps de nombres. $\mathrm{Au}$ cours de ses recherches, une nouvelle méthode d'approximation diophantienne - dite méthode des pentes - a été élaborée. Cet article propose une généralisation de ces travaux à une classe plus large de fibrés vectoriels, dits adéliques, définis sur un corps global. Ces fibrés possèdent aux places archimédiennes des normes qui ne sont plus nécessairement hermitiennes. Nous examinons également le lien avec la théorie des minima successifs adéliques. Pour parvenir à ces résultats, nous avons recours à plusieurs concepts de géométrie des espaces de Banach de dimension finie.

ABstRaCT - At the end of the twentieth century, J.-B. Bost developped a slope theory of hermitian vector bundles over number fields. A new method of diophantine approximation, the so-called slope method, has emerged from his research. Our article proposes a generalisation to adelic vector bundles over global fields. The norms at the archimedean places are no longer supposed to be hermitian. The link with adelic successive minima is also mentioned. To get these results, we use several concepts from the geometry of finite dimensional Banach spaces.

\section{Introduction.}

Ce texte décrit une généralisation de la théorie des pentes des fibrés vectoriels hermitiens sur l'anneau des entiers d'un corps de nombres aux fibrés vectoriels adéliques sur un corps global.

Étant donné un corps de nombres $k$ d'anneau des entiers $\mathcal{O}_{k}$, un fibré vectoriel hermitien $\bar{E}$ sur Spec $\mathcal{O}_{k}$ est la donnée d'un $\mathcal{O}_{k}$-module projectif

(*) Indirizzo dell'A.: Université Grenoble I, Institut Fourier, UMR 5582, BP 74, 38402 Saint-Martin-d'Hères Cedex, France.

E-mail: Eric.Gaudron@ujf-grenoble.fr 
de type fini $E$ et, pour toute place archimédienne $v$ de $k$, d'une norme $\|.\|_{v}$ sur l'espace vectoriel $E_{v}:=E \otimes_{k} k_{v}\left(k_{v}=\mathbf{R}\right.$ ou $\left.\mathbf{C}\right)$ qui est euclidienne si $v$ est une place réelle et qui est hermitienne, invariante par conjugaison complexe, si $v$ est une place complexe. À une telle donnée, l'on peut associer un nombre réel appelé degré d'Arakelov (et noté $\widehat{\operatorname{deg}}_{\mathrm{n}} \bar{E}$ ), qui, au signe près, mesure une «hauteur de $\bar{E}$ ». Ces nombres jouent un rôle important en géométrie d'Arakelov. Ce sont les éléments primitifs à partir desquels se bâtissent d'autres invariants associés à $\bar{E}$. Il s'agit notamment des pentes du graphe du polygone qui délimite supérieurement l'enveloppe convexe des couples de nombres réels $\left(\operatorname{rg} F, \widehat{\operatorname{deg}}_{\mathrm{n}} \bar{F}\right)$ où $\bar{F}$ parcourt les sousfibrés de $\bar{E}$. Les images de ces pentes par la fonction $x \mapsto e^{-x}$ se comparent aux minima successifs adéliques de $\bar{E}$, définis par Bombieri \& Vaaler [2]. À l'occasion de cours de $3^{\text {ème }}$ cycle donnés à l'Institut Henri Poincaré (Paris) en 1997 et 1999, J.-B. Bost a effectué une étude systématique des propriétés de ces nombres, élaborant ainsi une véritable théorie des pentes. L'objectif poursuivi initialement était de reformuler sous forme plus géométrique et intrinsèque la démonstration du théorème des périodes de D. Masser \& G. Wüstholz (voir [6]). Les notes de ces cours n'ont pas été publiées. Néanmoins plusieurs fragments se trouvent dans les articles $[6,7$, 18, 18, 19, 39]. De l'article fondateur [6] est issue une méthode - dite méthode des pentes - destinée à prouver des énoncés de transcendance et d'approximation diophantienne. Elle se rapproche de la méthode des déterminants d'interpolation de M. Laurent. La grande force de la méthode des pentes est de s'adapter naturellement à un problème de nature géométrique. Cette caractéristique renforce la clarté de l'argumentation en séparant distinctement les contributions, tout en faisant ressortir les invariants naturels des objets géométriques. Par exemple, elle a permis de mettre en lumière et de démontrer un critère d'algébricité de feuilles formelles (voir [7]). Nous l'avons également utilisée pour fournir des minorations de formes linéaires de logarithmes de variétés abéliennes principalement polarisées, minorations qui sont totalement explicites en la dimension et la hauteur de Faltings de la variété (voir [16]).

À l'usage, il arrive parfois que le cadre des fibrés vectoriels hermitiens sur Spec $\mathcal{O}_{k}$ dans lequel s'applique la méthode des pentes s'avère trop rigide. À la suite des travaux de S. Zhang [41], V. Maillot [22] ou bien encore de R. Rumely et al. [28], il est apparu que, si l'on souhaite construire une «hauteur canonique» sur les cycles d'une variété projective $X$ munie d'un fibré en droites ample $M$, les métriques que l'on doit mettre sur $M$ ne sont pas en général hermitiennes mais seulement continues. Cela empêche alors de mettre en œuvre la méthode des pentes telle quelle, comme on 
aimerait le faire par exemple avec l'espace vectoriel des sections globales $\mathrm{H}^{0}(X, M)$.

Ces observations nous ont amené à examiner à nouveau le formalisme des pentes pour des fibrés vectoriels munis d'une structure plus souple que celle des fibrés vectoriels hermitiens sur Spec $\mathcal{O}_{k}$. Avant de présenter plus en détail les résultats de cet article, je tiens à souligner que la plupart d'entre eux - preuves comprises - proviennent des cours de J.-B. Bost mentionnés ci-dessus, au moins en ce qui concerne le cas hermitien.

Dorénavant, nous considérons un corps global $k$ (corps de nombres ou corps de fonctions). En nous inspirant de [28], nous définissons la notion de fibré vectoriel adélique sur Spec $k$ qui généralise celle de fibré hermitien sur Spec $\mathcal{O}_{k}$. Un tel objet est la donnée d'un $k$-espace vectoriel $E$ de dimension finie $n$, muni d'une $k$-base e et, pour chaque place $v$ de $k$, d'une norme $\|\cdot\|_{v}$ sur $E \otimes_{k} \mathbf{C}_{v}$, invariante sous l'action des automorphismes continus de $\operatorname{Gal}\left(\mathbf{C}_{v} / k_{v}\right)$. On le note $\bar{E}=\left(E,\left(\|\cdot\|_{v}\right)_{v}\right)$. Si $v$ est ultramétrique, la norme $\|\cdot\|_{v}$ doit vérifier l'inégalité ultramétrique usuelle et, sauf pour un nombre fini de $v$, elle est égale à la norme du sup sur $E \otimes_{k} \mathbf{C}_{v}$, cet espace étant identifié à $\mathbf{C}_{v}^{n}$ au moyen de la base $\mathbf{e}$. Le trait marquant de ces fibrés adéliques est que les normes aux places archimédiennes ne sont plus nécessairement hermitiennes. La collection des normes de $\bar{E}$ dote l'espace adélique $E \otimes_{k} k_{\mathrm{A}}$ d'une boule unité, dont le logarithme du volume jouera le rôle de degré d'Arakelov de $\bar{E}$ (à une constante près). Il sera appelé degré adélique de $\bar{E}$ dans la suite. Une fois ces définitions fixées, nous étudions les propriétés de ce degré vis-à-vis des opérations usuelles que l'on peut effectuer sur l'ensemble des fibrés vectoriels adéliques (extension du corps de base, somme directe, etc.). Puis nous construisons l'analogue du «polygone canonique» à partir duquel s'obtiennent les $n$ pentes de $E$. Le reste de l'article s'attache alors à étudier certaines propriétés de ces pentes, en particulier leur comportement par transformation linéaire, c.-à-d. lorsque deux fibrés $\bar{E}$ et $\bar{F}$ ont leurs espaces vectoriels sous-jacent reliés par une application linéaire. Ceci donne naissance à plusieurs inégalités - dites inégalités de pentes - dont l'une est au cœur de la méthode des pentes, évoquée au début de cette introduction.

La plupart des résultats que nous obtenons sont basés sur le même schéma de preuve. On commence par s'intéresser au cas hermitien, c.-à-d. au cas d'un fibré vectoriel adélique dont les normes aux places archimédiennes sont hermitiennes. Les démonstrations sont alors très proches de celles déjà connues pour les fibrés vectoriels hermitiens sur Spec $\mathcal{O}_{k}$. Le passage au cas général s'effectue au moyen d'une comparaison entre une norme quelconque $\|$.$\| sur \mathbf{R}^{n}$ et une norme euclidienne, en fai- 
sant intervenir la distance - dite de Banach-Mazur - entre $\left(\mathbf{R}^{n},\|\|.\right)$ et l'espace euclidien usuel $\ell_{n}^{2}$. Cette démarche est fréquente dans l'étude de la géométrie des espaces de Banach de dimension finie (géométrie de Minkowski). Toutefois, un certain nombre d'adaptations et de reformulations dans le cadre adélique ont été nécessaires. Par exemple, nous définissons les fibrés vectoriels adéliques de John et Löwner associés à un fibré vectoriel adélique $\bar{E}$ sur Spec $k$, qui sont des fibrés vectoriels hermitiens qui encadrent au mieux $\bar{E}$ (en termes de volumes de boules unités). Ces fibrés fournissent des formules exactes pour le degré adélique de $\bar{E}$. Et cela conduit de temps en temps à des résultats plus fins, qui font intervenir le «quotient volumique adélique» de $\bar{E}$, qui est un nombre réel construit à l'aide du fibré de John de $\bar{E}$, au lieu de la distance de Banach-Mazur adélique. Un aspect intéressant de cette approche est que les termes d'erreurs induits sont très bien contrôlés. Ils sont bornés par une fonction

explicite de la dimension de $\bar{E}$ et du degré de $k$. De plus, ils disparaissent lorsque $\bar{E}$ est hermitien. Ceci assure que les énoncés établis dans cet article sont des généralisations du cas «classique», hermitien sur Spec $\mathcal{O}_{k}$.

Pour conclure, mentionnons que la théorie des pentes sous sa forme originelle revêt un aspect assez élémentaire à la fois en ce qui concerne les énoncés et les preuves. Afin de préserver son caractère accessible au nonspécialiste, nous avons rappelé quelques rudiments de théorie des adèles et de géométrie de Minkowski. Nous ne supposons de la part du lecteur aucune connaissance particulière relative à la théorie des pentes «classique». Les démonstrations sont données dans leur intégralité, fait susceptible d'entraîner çà et là des répétitions.

Remerciements. Je remercie Gaël Rémond de sa lecture attentive et critique d'une première version de ce texte et des corrections qu'il m'a suggérées. Je remercie également le rapporteur de l'attention qu'il a prêtée à ce texte en me signalant de nombreuses erreurs et inexactitudes.

\section{Préliminaires.}

\section{1. - Adèles sur un corps global.}

Ce paragraphe présente quelques propriétés bien connues des corps globaux et il fixe quelques notations, utilisées dans la suite. Une étude systématique des corps globaux et de leurs propriétés se trouve dans les ouvrages de C. Chevalley [13] et de A. Weil [40]. 
Soit $k$ un corps global. Il y a deux cas de figure selon la caractéristique de $k$.

\section{(1) La caractéristique de $k$ est nulle.}

Dans ce cas, le corps $k$ est un corps de nombres. On pose $k_{0}:=\mathbf{Q}$ et $D=\left[k: k_{0}\right]$ le degré absolu de $k$. On désigne par $|\cdot|_{v}$ la valeur absolue sur le complété $k_{v}$ (ou $\mathbf{C}_{v}$ ) de $k$ en la place $v$, normalisée de la manière suivante.

1) Si $v$ est archimédienne, $|\cdot|_{v}$ est la valeur absolue usuelle sur $\mathbf{R}$ ou $\mathbf{C}$.

2) Si $v$ est ultramétrique, de caractéristique résiduelle $p_{v}$, on a $\left|p_{v}\right|_{v}=p_{v}^{-1}$.

(2) La caractéristique de $k$ est $p>0$.

Le corps $k$ est une extension finie de $k_{0}:=\mathbf{F}_{p}(T)$. On note $D:=\left[k: k_{0}\right]$. Une place $v$ de $k$ est nécessairement ultramétrique et la place $v_{0}$ de $k_{0}$ correspondante est de deux sortes : soit elle provient d'un polynôme irréductible $\pi$ de $\mathbf{F}_{p}[T]$, soit l'idéal premier associé à $v_{0}$ est engendré par $T^{-1}$. Dans ce second cas la place $v_{0}$ est dite infinie, cette désignation étant bien sûr fonction du choix de $T$. Sur le complété $\left(k_{0}\right)_{v_{0}}$, on considère la valeur absolue $|.|_{v_{0}}$ normalisée par $|\pi|_{v_{0}}=p^{-\operatorname{deg} \pi}$ (premier cas) ou $|T|_{v_{0}}=p$ (second cas). On prolonge alors cette valeur absolue à $k_{v}$ de telle manière à ce que $|x|_{v}=|x|_{v_{0}}$ pour $x \in\left(k_{0}\right)_{v_{0}}$. Autrement dit, si $\mathrm{N}$ désigne l'application norme de l'extension $k_{v} \mid\left(k_{0}\right)_{v_{0}}$, on a $|x|_{v}=|\mathrm{N}(x)|_{v_{0}}^{1 / n_{v}}$ où $n_{v}$ est le degré local $\left[k_{v}:\left(k_{0}\right)_{v_{0}}\right]$.

Avec ces normalisations, si l'on pose $n_{v}:=1,2,\left[k_{v}: \mathbf{Q}_{p_{v}}\right],\left[k_{v}:\left(k_{0}\right)_{v_{0}}\right]$ selon que $v$ est réelle, complexe, ultramétrique (carac $k=0$ ou $p$ respectivement), l'application $x \in k_{v} \mapsto|x|_{v}^{n_{v}}$ est le module de Haar normalisé en la place $v$ et la formule du produit s'écrit alors

$$
\forall x \in k \backslash\{0\}, \quad \prod_{v \text { place de } k}|x|_{v}^{n_{v}}=1
$$

(dans ce produit tous les termes sauf un nombre fini valent 1 ). Si $K$ est une extension finie de $k$, il n'y a qu'un nombre fini de places $w$ de $K$ au-dessus d'une place $v$ de $k$. On dispose d'un isomorphisme de $K$-algèbres topologiques pour les complétés

$$
K \otimes_{k} k_{v} \simeq \prod_{w \mid v} K_{w}
$$


qui conduit en particulier à l'égalité des degrés

$$
[K: k]=\sum_{w \mid v}\left[K_{w}: k_{v}\right]
$$

(voir le chapitre 4 de [13]).

Soit $k_{\mathrm{A}}$ l'anneau des adèles de $k$. En tant que groupe localement compact, $\left(k_{\mathrm{A}},+\right)$ possède une mesure de Haar, unique à multiplication par un nombre réel strictement positif près. Le plongement diagonal $k \hookrightarrow k_{\mathrm{A}}$ confère à $k$ une structure de réseau dans $k_{\mathrm{A}}$ et l'espace compact $k_{\mathrm{A}} / k$ a une mesure de Haar finie. Plus généralement il en est de même pour $E \hookrightarrow E \otimes k_{\mathrm{A}}=: E_{\mathrm{A}}$ où $E$ est un $k$-espace vectoriel de dimension finie. La mesure dite de Tamagawa est celle pour laquelle la mesure du quotient $E_{\mathrm{A}} / E$ vaut 1.

- Si $k$ est un corps de nombres, soit $\mu_{v}$ la mesure de Haar définie sur le complété $k_{v}$ de $k$ en une place $v$ de la manière suivante:

a) Si $v$ est réelle, $\mu_{v}$ est la mesure de Lebesgue usuelle sur $\mathbf{R}$.

b) Si $v$ est complexe, $\mu_{v}$ s'identifie au double $2 \mathrm{~d} x \mathrm{~d} y$ de la mesure de Lebesgue sur $\mathbf{R}^{2}$.

c) Si $v$ est ultramétrique, on pose $\mu_{v}\left(\mathcal{O}_{v}\right)=1$ où $\mathcal{O}_{v}$ est l'anneau de valuation de $k_{v}$.

La mesure produit $\mu=\prod \mu_{v}$ est une mesure de Haar sur $k_{\mathrm{A}}$ pour laquelle la mesure de l'espace quotient $k_{\mathrm{A}} / k$ (c.-à-d. la mesure d'un domaine fondamental de celui-ci) égale $\left|D_{k}\right|^{1 / 2}$ où $D_{k}$ est le discriminant absolu de $k$ (proposition 7 du chapitre 5 de [40]).

- Si $k$ est un corps de fonctions, on note $\mu$ la mesure de Haar sur $k_{\mathbf{A}}$ telle que $\mu\left(\prod_{v} \mathcal{O}_{v}\right)=1$. On a alors $\mu\left(k_{\mathbf{A}} / k\right)=q^{g(k)-1}$ où $g(k) \in \mathbf{N}$ est le genre de $k$ et $q$ désigne le cardinal du plus grand corps fini inclus dans $k$ (corollaire 1 du chapitre 6 , ibid.).

Plus généralement, si $E$ est un $k$-espace vectoriel de dimension finie, le choix d'une $k$-base de $E$ fournit un isomorphisme $E \otimes k_{\mathbf{A}} \simeq k_{\mathbf{A}}^{\operatorname{dim} E}$ et une mesure de Haar $\mu_{E_{\mathrm{A}}}$ sur $E_{\mathrm{A}}$. Cette mesure ne dépend pas du choix de la base de $E$.

Avec ces normalisations, pour toute place $v$ de $k$ et tout nombre réel $r \in\left|k_{v}\right|_{v}$, on a

$$
\mu_{v}\left(\left\{x \in k_{v} ;|x|_{v} \leq r\right\}\right)=r^{n_{v}} \mu_{v}\left(\left\{x \in k_{v} ;|x|_{v} \leq 1\right\}\right) .
$$

Cette égalité reste valide si l'on remplace $\mu_{v}$ par une mesure de Haar quelconque sur $\left(k_{v},+\right)$. Enfin, il est commode de définir pour un adèle 
$a=\left(a_{v}\right)_{v} \in k_{\mathrm{A}}$ la valeur absolue adélique de $a$ comme le nombre réel

$$
|a|_{\mathbf{A}}:=\prod_{v \text { place de } k}\left|a_{v}\right|_{v}^{n_{v}} .
$$

Si $k$ est un corps de nombres, l'image par $|\cdot|_{\mathbf{A}}$ des idèles $k_{\mathbf{A}}^{\times}$de $k_{\mathbf{A}}$ est $\mathbf{R}_{+}^{*}$. Si $k$ est un corps de fonctions, l'image $\left|k_{\mathbf{A}}^{\times}\right|_{\mathbf{A}}$ est $\left\{q^{n} ; n \in \mathbf{Z}\right\}$ (voir [40], chap. $7, \S 5$, corollaire 6 ).

\section{2. - Géométrie de Minkowski.}

Pour comprendre les propriétés d'une norme quelconque sur $\mathbf{R}^{n}$, une possibilité est d'effectuer une comparaison avec la norme de $\ell_{n}^{p}$, $p \in[1,+\infty]$, et, si possible, avec la norme euclidienne de $\ell_{n}^{2}$. La géométrie de Minkowski est l'étude des $\mathbf{R}$-espaces vectoriels normés $(E,\|\|$.$) de di-$ mension finie. L'un des axes de cette étude consiste précisément à s'intéresser à la structure euclidienne qui se rapproche le plus de la structure d'espace vectoriel normé de $E$, en un sens que nous préciserons un peu plus loin. Dans le cas d'un corps de nombres, cette approche s'avérera être la clef qui permet d'aborder la théorie des fibrés vectoriels adéliques.

Pour écrire cette synthèse, nous avons consulté les ouvrages de G. Pisier [23], R. Ryan [29] et A. Thompson [37] ainsi que les articles [10, 26, 30].

Dans tout ce paragraphe, $E$ est un R-espace vectoriel de dimension $n \geq 1$, muni d'une mesure de Haar vol. L'espace vectoriel dual $E^{\vee}=\operatorname{Hom}_{\mathbf{R}}(E, \mathbf{R})$ possède alors une mesure de Haar particulière vol*, associée au choix de vol, caractérisée de la manière suivante: soit $\left(e_{1}, \ldots, e_{n}\right)$ une base de $E$ et le parallélotope

$$
\mathscr{P}:=\left\{x_{1} e_{1}+\cdots+x_{n} e_{n} ; 0 \leq x_{i} \leq 1\right\} .
$$

De même, on dispose de la base duale $\left(e_{1}^{*}, \ldots, e_{n}^{*}\right)$ et du parallélotope dual $\mathscr{P}^{*}$ associé. Alors vol ${ }^{*}$ est l'unique mesure de Haar telle que

$$
\operatorname{vol}(\mathscr{P}) \operatorname{vol}^{*}\left(\mathscr{P}^{*}\right)=1 \text {. }
$$

Soit $C \subseteq E$ une partie convexe, d'intérieur non vide, compact et symétrique par rapport à l'origine. Un tel ensemble est appelé corps convexe (symétrique $\left.{ }^{*}\right)$ ). La fonction jauge

$$
\forall x \in E, \quad j(x):=\inf \left\{\lambda>0 ; \frac{x}{\lambda} \in C\right\}
$$

(*) Tous les corps convexes considérés dans ce texte sont symétriques et nous omettrons de le préciser à chaque fois. 
fait le lien entre une norme sur $E$ et le corps convexe qui est la boule unité pour cette norme. Autrement dit, le couple $(E, C)$ est la donnée d'une structure normée $(E, j)$ sur l'espace vectoriel $E$. Dans la suite, nous noterons aussi $\|$.$\| la norme j$ sur $E$.

DÉfinition 2.1. Le polaire de $C$, noté $C^{\circ}$, est l'ensemble $C^{\circ}:=\left\{\varphi \in E^{\vee} ;|\varphi(C)| \subseteq[0,1]\right\}$.

On vérifie que $C^{\circ}$ est la boule unité fermée de l'espace dual $\left(E^{\mathrm{v}}, j^{\mathrm{v}}\right)$. Lorsque $p \in[1,+\infty]$, on note $b_{n}^{p}$ (ou $b_{n, \mathbf{R}}^{p}$ ) la boule unité fermée de l'espace de Banach $\ell_{n}^{p}:=\left(\mathbf{R}^{n},|\cdot|_{p}\right)$, où

$$
\left|\left(x_{1}, \ldots, x_{n}\right)\right|_{p}= \begin{cases}\left(\left|x_{1}\right|^{p}+\cdots+\left|x_{n}\right|^{p}\right)^{1 / p} & \text { si } p \in[1,+\infty[ \\ \max \left\{\left|x_{1}\right|, \ldots,\left|x_{n}\right|\right\} & \text { si } p=+\infty .\end{cases}
$$

Si $p \in] 0,1\left[\right.$ et $n \geq 2$, cette application $|\cdot|_{p}$ n'est plus une norme et $b_{n}^{p}$ n'est plus convexe, mais cela reste un ensemble mesurable au sens de Lebesgue. Ainsi, pour tout $p>0$ et pour la mesure de Lebesgue $\operatorname{vol}_{n} \operatorname{sur} \mathbf{R}^{n}$, on a

$$
\operatorname{vol}_{n}\left(b_{n}^{p}\right)=\frac{\left(2 \Gamma\left(1+\frac{1}{p}\right)\right)^{n}}{\Gamma\left(1+\frac{n}{p}\right)}
$$

où $\Gamma(x):=\int_{0}^{+\infty} t^{x-1} e^{-t} \mathrm{~d} t$ (voir formule (9) un peu plus loin). De même, $b_{n, \mathbf{C}}^{p}$ désigne la boule unité fermée pour la norme $|\cdot|_{p} \operatorname{sur} \mathbf{C}^{n}$. En identifiant $\mathbf{C}^{n}$ à $\mathbf{R}^{2 n}$, la mesure de Lebesgue de $b_{n, \mathbf{C}}^{p}$ vaut

$$
\operatorname{vol}_{2 n}\left(b_{n, \mathbf{C}}^{p}\right)=\left(\frac{\pi}{2}\right)^{n} \operatorname{vol}_{n}\left(b_{n, \mathbf{R}}^{p / 2}\right) .
$$

À un corps convexe $C$, l'on peut associer l'invariant suivant:

DÉFINITION 2.2. Le produit de Mahler du corps convexe $C$, noté $P(C)$, est le produit $\operatorname{vol}(C) \operatorname{vol}^{*}\left(C^{\circ}\right)$.

Ce nombre réel ne dépend pas du choix de la mesure de Haar vol sur $E$. De plus, il est invariant par isomorphisme: si $u \in \mathrm{GL}(E)$ alors $P(u(C))=P(C)$. C'est pourquoi l'on peut omettre la référence à $E$ dans la notation du produit de Mahler. 
ThÉORÈme 2.3. Pour tout corps convexe $C$, on a

$$
P(C) \leq P\left(b_{n}^{2}\right) \quad(\text { Blaschke-Santaló })
$$

et l'existence d'une constante absolue $c \in] 0,+\infty[$ telle que

$$
\left.P(C) \geq e^{-c n} P\left(b_{n}^{2}\right) \quad \text { (Bourgain-Milman }\right) .
$$

La première inégalité a été établie dans [1,31], articles auxquels on peut adjoindre le texte de J. Saint-Raymond [30] qui comporte une preuve «élémentaire» de ce résultat et qui démontre qu'il y a égalité seulement si, dans une certaine base de $E$, le convexe $C$ est la boule unité euclidienne usuelle (on dit alors que $C$ est un ellipsoïde). La seconde inégalité, démontrée dans [10], est plus difficile à obtenir. Une valeur explicite pour la constante $c$ n'est pas connue à l'heure actuelle. Aussi peut-il être utile de mentionner une minoration plus faible due à K. Mahler [21] :

$$
P(C) \geq \frac{4^{n}}{(n !)^{2}},
$$

minoration qui entraîne (*)

$$
P(C) \geq e^{-n \log (e n)} P\left(b_{n}^{2}\right) .
$$

Une preuve de la conjecture de Mahler $P(C) \geq 4^{n} / n$ ! permettrait d'obtenir une constante $c$ explicite dans l'inégalité de Bourgain-Milman.

Ellipsoïdes de John et Löwner.

Un ellipsoïde de $E$ est un ensemble de la forme $D=\{x \in E ; q(x) \leq 1\}$ où $q: E \rightarrow \mathbf{R}$ est une forme quadratique définie positive. Si l'on fixe une base de $E$ qui permet d'identifier $E$ à $\mathbf{R}^{n}$, un ellipsoïde est l'image de la boule unité euclidienne $b_{n}^{2}$ par un isomorphisme de $E$. En particulier, le produit de Mahler de $D$ est celui de $b_{n}^{2}$.

(*) Le calcul qui conduit à cette minoration est basé sur la formule donnée auparavant pour le volume de $b_{n}^{2}$ ainsi que sur l'existence d'une fonction $\eta$, décroissante, positive et nulle à l'infini, telle que

$$
\forall x>0, \quad \Gamma(1+x)=\sqrt{2 \pi x}\left(\frac{x}{e}\right)^{x} e^{\eta(x)}
$$

(voir [25], chap. 2, § 4). 
DÉFINITION-THÉORÈME 2.4. Étant donné un corps convexe (symétrique) $C$, il existe un unique ellipsoïde $J(C)$, appelé ellipsoïde de John, inclus dans $C$ et de volume maximal. De même, il existe un unique ellipsoïde $L(C)$, dit ellipsö̈de de Löwner, contenant $C$ et de volume minimal.

La seconde assertion découle de la première par dualité: $J(C)^{\circ}=L\left(C^{\circ}\right)$, car $P(J(C))$ est constant, égal à $P\left(b_{n}^{2}\right)$. L'unicité est le point difficile et remarquable de cet énoncé. Elle entraine les inclusions $C \subseteq \sqrt{n} J(C)$ et $L(C) \subseteq \sqrt{n} C$ (ce n'est pas immédiat, voir [37], p. 84). En notant $|\cdot|_{J(C)}$ (resp. $\left.|\cdot|_{L(C)}\right)$ la norme euclidienne sur $E$ associée à $J(C)(\operatorname{resp} . L(C))$, cela se traduit par les inégalités

$$
\forall x \in E, \quad \frac{1}{\sqrt{n}}|x|_{J(C)} \leq j(x) \leq|x|_{J(C)} \quad \text { et } \quad|x|_{L(C)} \leq j(x) \leq \sqrt{n}|x|_{L(C)} .
$$

On dispose ainsi de deux structures euclidiennes qui encadrent la norme donnée sur $E$.

\section{Quotient volumique.}

Ce paragraphe emprunte beaucoup au texte [26] de M. Rogalski. On note $\mathbf{B}(E,\|\|$.$) la boule unité fermée de l'espace vectoriel normé (E,\|\|$.$) .$

DÉFINITION 2.5. Le quotient volumique $\left.{ }^{*}\right)$ de $(E,\|\|$.$) , noté vr (E)$, est le nombre réel $\geq 1$ défini par

$$
\operatorname{vr}(E):=\inf \left\{\left(\frac{\operatorname{vol}(\mathbf{B}(E,\|\cdot\|))}{\operatorname{vol}(D)}\right)^{1 / n} ; D \text { ellipsoïde } \subseteq \mathbf{B}(E,\|.\|)\right\} .
$$

La définition même de l'ellipsoïde John entraîne

$$
\operatorname{vr}(E)=\left(\frac{\operatorname{vol}(\mathbf{B}(E,\|\cdot\|))}{\operatorname{vol}(J(\mathbf{B}(E,\|\cdot\|)))}\right)^{1 / n}
$$

et l'inclusion $C \subseteq \sqrt{n} J(C)$ donne alors vr $(E) \leq \sqrt{n}$. Dans cette inégalité, la fonction $n \mapsto \sqrt{n}$ ne peut pas être remplacée par une fonction $f$ telle que $f(n) / \sqrt{n} \underset{n \rightarrow+\infty}{\longrightarrow} 0$. En revanche, on peut montrer que $\operatorname{vr}(E) \leq \delta \sqrt{n}$ où $\delta \in] 0,1[$ est explicite (par exemple $\delta=0,95$ ). M. Rogalski a calculé le

(*) Volume ratio en anglais. 
quotient volumique de $\ell_{n}^{p}$ :

$$
\operatorname{vr}\left(\ell_{n}^{p}\right)=\Phi_{p}(n) n^{\max \left\{0,\left(\frac{1}{2}-\frac{1}{p}\right)\right\}} \sqrt{\frac{2}{\pi}} \Gamma\left(1+\frac{1}{p}\right) e^{\frac{1}{p}-\frac{1}{2}} p^{\frac{1}{p}}
$$

où $\Phi_{p}(n) \in[1 / 3,2]$ et $\Phi_{p}(n) \underset{n \rightarrow+\infty}{\longrightarrow} 1$.

Nous aurons également besoin de la variante avec l'ellipsoïde de Löwner.

DÉFINition 2.6. On note $\widetilde{\operatorname{vr}}(E)$ le nombre réel $\geq 1$ défini par

$$
\widetilde{\operatorname{vr}}(E):=\sup \left\{\left(\frac{\operatorname{vol}(D)}{\operatorname{vol}(\mathbf{B}(E,\|\cdot\|))}\right)^{1 / n} ; \mathbf{B}(E,\|.\|) \subseteq D \text { ellipsoïde }\right\} .
$$

On a done

$$
\widetilde{\operatorname{vr}}(E)=\left(\frac{\operatorname{vol}(L(\mathbf{B}(E,\|\cdot\|)))}{\operatorname{vol}(\mathbf{B}(E,\|\cdot\|))}\right)^{1 / n} \text { et } \quad \widetilde{\operatorname{vr}}(E) \leq \sqrt{n} .
$$

Distance de Banach-Mazur.

Définition 2.7. Étant donné deux corps convexes $C_{1}$ et $C_{2}$ de $E$, la distance $\mathrm{d}\left(C_{1}, C_{2}\right)$ entre ces deux ensembles est

$$
\mathrm{d}\left(C_{1}, C_{2}\right):=\inf \left\{a b ; \quad a>0, b>0, \quad C_{1} \subseteq a C_{2} \quad \text { et } \quad C_{2} \subseteq b C_{1}\right\} .
$$

La distance - dite de Banach-Mazur - entre les espaces de Banach $E_{1}=\left(E, C_{1}\right)$ et $E_{2}=\left(E, C_{2}\right)$ est

$$
\mathrm{d}\left(E_{1}, E_{2}\right):=\inf \left\{\mathrm{d}\left(C_{1}, u\left(C_{2}\right)\right) ; \quad u \in \mathrm{GL}(E)\right\} .
$$

Plus généralement, la distance de Banach-Mazur entre deux espaces de Banach $E$ et $F$ de même dimension (finie) est d $(E, \varphi(F))$ où $\varphi: F \rightarrow E$ est un isomorphisme quelconque entre $F$ et $E$.

Cette dernière quantité ne dépend pas du choix de $\varphi$. La terminologie «distance» se justifie par l'inégalité $\mathrm{d}\left(E_{1}, E_{3}\right) \leq \mathrm{d}\left(E_{1}, E_{2}\right) \mathrm{d}\left(E_{2}, E_{3}\right)$ où $E_{1}, E_{2}, E_{3}$ sont des espaces vectoriels normés de même dimension. La distance qui nous intéressera le plus dans la suite est celle entre $(E,\|\|$.$) et$ $\ell_{n}^{2}$. Par définition même de cette distance, pour tout $\varepsilon>0$, il existe une norme euclidienne $|\cdot|_{\varepsilon}$ sur $E$ telle que, pour tout $x \in E$, on ait

$$
\forall x \in E, \quad|x|_{\varepsilon} \leq\|x\| \leq \mathrm{d}\left(E, \ell_{n}^{2}\right)(1+\varepsilon)|x|_{\varepsilon} .
$$


On vérifie alors l'encadrement

$$
1 \leq \operatorname{vr}(E) \operatorname{vr}\left(E^{\vee}\right) \leq \mathrm{d}\left(E, \ell_{n}^{2}\right) \leq \sqrt{n},
$$

sans qu'il y ait égalité en général. En réalité l'on peut même exhiber une suite d'espaces normés $E_{n}$ de dimension $n$ telle que

$$
\mathrm{d}\left(E_{n}, \ell_{n}^{2}\right) / \operatorname{vr}\left(E_{n}\right) \underset{n \rightarrow+\infty}{\longrightarrow}+\infty .
$$

On a aussi $1 \leq \widetilde{\operatorname{vr}}(E) \widetilde{\operatorname{vr}}\left(E^{\vee}\right) \leq \mathrm{d}\left(E, \ell_{n}^{2}\right)$.

Somme directe.

Soit $E, F$ deux espaces vectoriels normés de dimensions respectives $n$ et $m$. Soit $\varsigma$ une norme symétrique sur $\mathbf{R}^{2}$, invariante par changement de signes sur les coordonnées, telle que $\varsigma(1,0)=\varsigma(0,1)=1$. On munit la somme directe $E \oplus F$ de la norme $\|(x, y)\|_{E \oplus_{s} F}:=\varsigma\left(\|x\|_{E},\|y\|_{F}\right)$. L'espace vectoriel normé obtenu sera noté $E \oplus_{\varsigma} F$. On vérifie

(8) $\forall x \in E, \forall y \in F, \quad \max \left\{\|x\|_{E},\|y\|_{F}\right\} \leq\|(x, y)\|_{E \oplus_{S} F} \leq\|x\|_{E}+\|y\|_{F}$.

Proposition 2.8. Avec les données ci-dessus, soit $\operatorname{vol}_{E}$ (resp. $\operatorname{vol}_{F}$ ) une mesure de Haar sur $E$ (resp. F). On dispose de la mesure produit $\operatorname{vol}_{E \oplus F}:=\operatorname{vol}_{E} \otimes \operatorname{vol}_{F}$ sur $E \oplus F \simeq E \times F$. On a alors

$$
\left(\begin{array}{c}
n+m \\
n
\end{array}\right)^{-1} \leq \frac{\operatorname{vol}_{E \oplus F}\left(\mathbf{B}\left(E \oplus F,\|\cdot\|_{E \oplus_{G} F}\right)\right)}{\operatorname{vol}_{E}\left(\mathbf{B}\left(E,\|\cdot\|_{E}\right)\right) \operatorname{vol}_{F}\left(\mathbf{B}\left(F,\|\cdot\|_{F}\right)\right)} \leq 1 .
$$

DÉmonstration. L'inégalité de droite est une simple conséquence de l'inclusion

$$
\mathbf{B}\left(E \oplus F,\|\cdot\|_{E \oplus_{s} F}\right) \subseteq \mathbf{B}\left(E,\|\cdot\|_{E}\right) \times \mathbf{B}\left(F,\|\cdot\|_{F}\right),
$$

qui résulte de la majoration $\max \left\{\|x\|_{E},\|y\|_{F}\right\} \leq\|(x, y)\|_{E \oplus_{G} F}$. L'autre inégalité repose sur la formule intégrale

$$
\forall p>0, \quad \operatorname{vol}_{E}\left(\mathbf{B}\left(E,\|\cdot\|_{E}\right)\right) \times \Gamma\left(1+\frac{n}{p}\right)=\int_{E} e^{-\|x\|_{E}^{p}} \mathrm{~d}\left(\operatorname{vol}_{E}\right)(x),
$$

qui s'obtient grâce au théorème de Fubini en intégrant $\left(e^{-t} \mathrm{~d} t\right) \otimes \mathrm{d}\left(\operatorname{vol}_{E}\right)$ sur l'ensemble $\left\{(t, x) ; t \geq\|x\|_{E}^{p}\right\} \subseteq \mathbf{R} \times E$. On applique cette formule à $E \oplus_{\varsigma} F$ 
et $p=1$. De la majoration $\|(x, y)\|_{E \oplus_{\xi} F} \leq\|x\|_{E}+\|y\|_{F}$ découle l'inégalité

$$
\operatorname{vol}_{E \oplus F}\left(\mathbf{B}\left(E \oplus F,\|\cdot\|_{E \oplus_{\zeta} F}\right)\right)(n+m) ! \geq \int_{E \oplus F} e^{-\|x\|_{E}-\|y\|_{F}} \mathrm{~d}\left(\operatorname{vol}_{E \oplus F}\right)(x, y),
$$

et la dernière intégrale vaut exactement

$$
\left(\operatorname{vol}_{E}\left(\mathbf{B}\left(E,\|\cdot\|_{E}\right)\right) n !\right)\left(\operatorname{vol}_{F}\left(\mathbf{B}\left(F,\|\cdot\|_{F}\right)\right) m !\right) .
$$

Ceci conclut la démonstration.

Remarque 2.9. Si $\varsigma(\alpha, \beta)=\left(|\alpha|^{p}+|\beta|^{p}\right)^{1 / p}, \quad p \geq 1$, la formule intégrale (9) fournit l'égalité

$$
\frac{\operatorname{vol}_{E \oplus F}\left(\mathbf{B}\left(E \oplus F,\|\cdot\|_{E \oplus_{\zeta} F}\right)\right)}{\operatorname{vol}_{E}\left(\mathbf{B}\left(E,\|\cdot\|_{E}\right)\right) \operatorname{vol}_{F}\left(\mathbf{B}\left(F,\|\cdot\|_{F}\right)\right)}=\frac{\Gamma\left(1+\frac{n}{p}\right) \Gamma\left(1+\frac{m}{p}\right)}{\Gamma\left(1+\frac{n+m}{p}\right)} .
$$

Autrement dit, le quotient des volumes ne dépend que des dimensions de $E$ et de $F$.

Normes tensorielles.

Sur le produit tensoriel de deux espaces vectoriels normés de dimension finie coexistent de nombreuse normes, que l'on peut obtenir de manière «naturelle» à partir des normes sur les espaces de départ. Les conditions minimales que nous exigerons pour une telle norme sur le produit tensoriel sont les suivantes.

DÉFInITION 2.10. Soit $\ell$ un entier $\geq 1$. Une norme tensorielle $\left(^{*}\right)$ d'ordre $\ell$ est la donnée pour tous espaces de Banach $\left(E_{i},\|\cdot\|_{E_{i}}\right)_{1 \leq i \leq \ell}(\operatorname{sur} \mathbf{R}$ ou $\mathbf{C})$ de dimension finie d'une norme $\alpha\left(\cdot ; E_{1}, \ldots, E_{\ell}\right)$ sur le produit tensoriel $E_{1} \otimes \cdots \otimes E_{\ell}$ telle que :

(i) pour tout $i \in\{1, \ldots, \ell\}$, pour tout $e_{i} \in E_{i}$, on a

$$
\alpha\left(e_{1} \otimes \cdots \otimes e_{\ell} ; E_{1}, \ldots, E_{\ell}\right) \leq \prod_{i=1}^{\ell}\left\|e_{i}\right\|_{E_{i}},
$$

(*) (Finitely generated) uniform crossnorm, selon la terminologie de Schatten (voir [29], chap. 6). 
(ii) la famille $\alpha=\left(\alpha\left(\cdot ; E_{1}, \ldots, E_{\ell}\right)\right)_{\left(E_{1}, \ldots, E_{\ell}\right)}$ doit vérifier : Pour tout $i \in\{1, \ldots, \ell\}$, pour tous espaces normés $E_{i}$ et $F_{i}$ de dimension finie et toutes applications linéaires $u_{i}: E_{i} \rightarrow F_{i}$, la norme d'opérateur de $u_{1} \otimes \cdots \otimes u_{\ell}: E_{1} \otimes \cdots \otimes E_{\ell} \rightarrow F_{1} \otimes \cdots \otimes F_{\ell}$ est plus petite que le produit des normes d'opérateur $\prod_{i=1}^{\ell}\left\|u_{i}\right\|$.

Une norme tensorielle hermitienne est une norme tensorielle qui restreinte aux espaces hermitiens est la norme hermitienne usuelle (*) sur le produit tensoriel. Nous noterons $E_{1} \otimes_{\alpha} \cdots \otimes_{\alpha} E_{\ell}$ ou $\otimes_{\alpha, i=1}^{\ell} E_{i}$ l'espace vectoriel $E_{1} \otimes \cdots \otimes E_{\ell}=: \otimes_{i=1}^{\ell} E_{i}$ muni de la norme $\alpha\left(\cdot ; E_{1}, \ldots, E_{\ell}\right)$.

On montre aisément que si les conditions (i) et (ii) ci-dessus sont réalisées alors il y a égalité : $\alpha\left(e_{1} \otimes \cdots \otimes e_{\ell}\right)=\prod_{i=1}^{\ell}\left\|e_{i}\right\|_{E_{i}}$ et $\left\|u_{1} \otimes \cdots \otimes u_{\ell}\right\|=$ $=\prod_{i=1}^{\ell}\left\|u_{i}\right\|$.

Examples 2.11. Normes de Chevet-Saphard (d'ordre 2). Soit $\left(E,\|\cdot\|_{E}\right)$ et $\left(F,\|\cdot\|_{F}\right)$ deux espaces vectoriels normés de dimension finie. Soit $\ell \in \mathbf{N} \backslash\{0\}$. Soit $p \in[1,+\infty]$ et $p^{\prime}$ son conjugué, c.-à-d. $1 / p+1 / p^{\prime}=1$. $\operatorname{Si}\left(e_{1}, \ldots, e_{\ell}\right) \in E^{\ell}$, on note

$$
\left\|\left(e_{1}, \ldots, e_{\ell}\right)\right\|_{p}:= \begin{cases}\left(\sum_{i=1}^{\ell}\left\|e_{i}\right\|_{E}^{p}\right)^{1 / p} & \text { si } 1 \leq p<\infty, \\ \max _{1 \leq i \leq \ell}\left\{\left\|e_{i}\right\|_{E}\right\} & \text { si } p=+\infty .\end{cases}
$$

De même, si $\left(f_{1}, \ldots, f_{\ell}\right) \in F^{\ell}$, on pose

$$
\left\|\left(f_{1}, \ldots, f_{\ell}\right)\right\|_{p}^{w}:=\sup \left\{\left\|\sum_{i=1}^{\ell} \lambda_{i} f_{i}\right\|_{F} ;\left(\lambda_{1}, \ldots, \lambda_{\ell}\right) \in b_{\ell}^{p}\right\}
$$

(le $w$ est l'initiale de weak). Alors la norme à gauche de Chevet-Saphard, notée $g_{p}$ ou $g_{p}(\cdot ; E, F)$, est la norme sur $E \otimes F$ définie par: pour tout $x \in E \otimes F$,

$$
g_{p}(x ; E, F):=\inf \left\{\left\|\left(e_{1}, \ldots, e_{\ell}\right)\right\|_{p}\left\|\left(f_{1}, \ldots, f_{\ell}\right)\right\|_{p^{\prime}}^{w} ; x=\sum_{i=1}^{\ell} e_{i} \otimes f_{i}\right\} .
$$

Cela définit une norme tensorielle d'ordre 2 (voir [29]) et, pour $p=2$, la norme $g_{2}$ est une norme tensorielle hermitienne au sens ci-dessus.

(*) Autrement dit, si $E$ et $F$ sont hermitiens, munis de bases orthonormées $\left(e_{1}, \ldots, e_{n}\right)$ et $\left(f_{1}, \ldots, f_{m}\right)$ respectivement, et si $x=\sum_{i, j} x_{i, j} e_{i} \otimes f_{j} \in E \otimes F$ alors $\alpha(x ; E, F)^{2}=\sum_{i, j}\left|x_{i, j}\right|^{2}$. 
Cet exemple et une simple récurrence permettent de construire des normes tensorielles (éventuellement hermitiennes) d'ordre quelconque.

L'observation suivante, bien que très simple, sera d'un usage constant dans la suite (le corps de base est $\mathbf{R}$ ou $\mathbf{C}$ ).

Proposition 2.12. Soit $\ell \in \mathbf{N} \backslash\{0\}$ et $\alpha$ une norme tensorielle d'ordre $\ell$. Pour tout $i \in\{1, \ldots, \ell\}$, soit $E_{i}^{\prime} \subseteq E_{i}$ des espaces vectoriels de dimension finie. Soit $|\cdot|_{E_{i}^{\prime}}$ et $|\cdot|_{E_{i}}$ deux normes sur $E_{i}^{\prime}$ et $E_{i}$ respectivement. Supposons que, pour tout $e_{i} \in E_{i}^{\prime}$, on a $\left|e_{i}\right|_{E_{i}} \leq\left|e_{i}\right|_{E_{i}^{\prime}}$. Alors, pour tout $e \in \otimes_{i=1}^{\ell} E_{i}^{\prime}$, on $a$

$$
\alpha\left(e ;\left(E_{1},|\cdot|_{E_{1}}\right), \ldots,\left(E_{\ell},|\cdot|_{E_{\ell}}\right)\right) \leq \alpha\left(e ;\left(E_{1}^{\prime},|\cdot|_{E_{1}^{\prime}}\right), \ldots,\left(E_{\ell}^{\prime},\left.|\cdot|\right|_{E_{\ell}^{\prime}}\right)\right) .
$$

DÉmonstration. Il s'agit de la condition (iii) caractérisant la norme tensorielle $\alpha$, appliquée aux morphismes d'inclusion $x \mapsto x$ de $\left(E_{i}^{\prime},|\cdot|_{E_{i}^{\prime}}\right)$ dans $\left(E_{i},|\cdot|_{E_{i}}\right)$ pour tout $i \in\{1, \ldots, \ell\}$.

Remarques 2.13. a) Le lecteur peu accoutumé prendra garde aux nombreuses chausse-trapes des normes tensorielles. Pour $\alpha$ d'ordre 2, la loi interne $(E, F) \mapsto E \otimes_{\alpha} F$ sur l'ensemble des espaces normés de dimension finie n'est en général ni associative ni commutative. De plus, si $F$ est un sous-espace vectoriel de $E$, la norme $\alpha(\cdot ; F, F)$ sur $F \otimes F$ n'est en général pas la restriction de la norme $\alpha(\cdot ; E, E)$ à $F \otimes F$ (c.-à-d. $F \otimes_{\alpha} F$ n'est pas un sous-Banach de $E \otimes_{\alpha} E$ ). Il en est de même pour un quotient $E \rightarrow G$ ou pour la dualité : la norme sur $\left(E^{\vee} \otimes_{\alpha} F^{\vee}\right)^{\vee}$ n'est en général pas égale à $\alpha(\cdot ; E, F)$.

b) Dans la suite, nous demanderons souvent aux normes tensorielles d'être hermitiennes. Au delà de la norme $g_{2}$ de l'exemple 2.11, il existe en réalité une infinité de telles normes tensorielles, non équivalentes entre elles, comme l'affirme un résultat de Schatten \& Puhl (voir [14], p. 357).

Cas d'un espace vectoriel complexe.

Dans ce paragraphe, $E$ est un $\mathbf{C}$-espace vectoriel de dimension $n \geq 1$. Soit $C \subseteq E$ un sous-ensemble convexe et compact, d'intérieur non vide. Afin que la jauge définisse une norme sur $E$ (en particulier pour que la relation $j\left(e^{i \theta} x\right)=j(x)$ soit vérifiée pour tous $\theta \in \mathbf{R}$ et $\left.x \in E\right)$, on suppose

$$
\forall \theta \in \mathbf{R}, \quad e^{i \theta} \cdot C=C .
$$

Notons $E_{\mathbf{R}}$ le $\mathbf{R}$-espace vectoriel sous-jacent à $E$ et $C_{\mathbf{R}}$ le sous-ensemble de $E_{\mathbf{R}}$ induit par $C$. Aux objets $E_{\mathbf{R}}$ et $C_{\mathbf{R}}$ l'on peut appliquer les résultats pré- 
cédents et, en particulier, l'on dispose des ellipsoïdes de John et Löwner associés à $C_{\mathbf{R}}$. Les normes euclidiennes sur $E_{\mathbf{R}}$ données par ces ellipsoïdes définissent des normes hermitiennes sur $E$. En effet l'hypothèse (11), l'unicité de ces ellipsoïdes et la conservation des volumes par les applications $x=x_{1}+i x_{2} \in E_{\mathbf{R}} \mapsto e^{i \theta} \cdot x=x_{1} \cos \theta-x_{2} \sin \theta+i\left(x_{1} \sin \theta+x_{2} \cos \theta\right) \in E_{\mathbf{R}}$ où $\theta \in \mathbf{R}$, entraînent

$$
e^{i \theta} \cdot J\left(C_{\mathbf{R}}\right)=J\left(C_{\mathbf{R}}\right) \quad \text { et } \quad e^{i \theta} \cdot L\left(C_{\mathbf{R}}\right)=L\left(C_{\mathbf{R}}\right) .
$$

Ainsi $J\left(C_{\mathbf{R}}\right)$ et $L\left(C_{\mathbf{R}}\right)$ définissent des ellipsoïdes complexes de $E$, c.-à-d. égaux à la boule unité fermée $b_{n, \mathbf{C}}^{2}$ de l'espace hermitien usuel $\left(\mathbf{C}^{n},|\cdot|_{2}\right)$, après le choix d'une base convenable de $E$. Comme dans le cas réel, on les note plus simplement $J(C)$ et $L(C)$. L'hypothèse (11) assure en réalité que tout se passe comme si l'on raisonnait dans $E_{\mathbf{R}} \simeq \mathbf{R}^{2 n}$. Le quotient volumique d'un C-espace vectoriel normé $(E,\|\|$.$) est défini par la formule$

$$
\operatorname{vr}(E)=\inf \left\{\left(\frac{\operatorname{vol}(\mathbf{B}(E,\|\cdot\|))}{\operatorname{vol}(D)}\right)^{\frac{1}{2 n}} ; \quad D \text { ellipsoïde complexe } \subseteq E\right\}
$$

où vol est une mesure de Haar sur $E$. Comme dans le cas réel, cette quantité est atteinte pour l'ellipsoïde de John $J(C)$. De même la définition de la distance de Banach-Mazur s'étend au cas complexe. Et l'on dispose de l'encadrement

$$
1 \leq \operatorname{vr}(E) \operatorname{vr}\left(E^{\vee}\right) \leq \mathrm{d}\left(E, \ell_{n, \mathbf{C}}^{2}\right) \leq \sqrt{2 n}=\sqrt{\operatorname{dim}_{\mathbf{R}} E} .
$$

\section{Fibré vectoriel adélique.}

Suivant en partie R. Rumely et al. [28], nous définissons ici une notion de fibré vectoriel adélique sur Spec $k$, qui généralise celle de fibré vectoriel hermitien sur $\operatorname{Spec} \mathcal{O}_{k}$, qui est à la base même de la géométrie d'Arakelov.

Soit $k$ un corps global et $v$ une place de $k$. On note $\mathbf{C}_{v}$ la complétion d'une clôture algébrique de $k_{v}$. Si $K$ est une extension finie de $k$ et $w$ une place de $K$ au-dessus de $v$, on a un isomorphisme topologique de corps valués $\mathbf{C}_{w} \simeq \mathbf{C}_{v}$. Soit $E$ un $k$-espace vectoriel. Une norme sur $E \otimes_{k} \mathbf{C}_{v}$ est une application $\|\cdot\|_{v}: E \otimes \mathbf{C}_{v} \rightarrow \mathbf{R}^{+}$qui satisfait aux trois conditions :

(i) $\forall x \in E \otimes \mathbf{C}_{v},\|x\|_{v}=0 \Longleftrightarrow x=0$.

(ii) $\forall x \in E \otimes \mathbf{C}_{v}, \forall \lambda \in \mathbf{C}_{v},\|\lambda x\|_{v}=|\lambda|_{v} \cdot\|x\|_{v}$.

(iii) $\forall x, y \in E \otimes \mathbf{C}_{v},\|x+y\|_{v} \leq\|x\|_{v}+\|y\|_{v}$. 
DÉFInITION 3.1. Un fibré vectoriel adélique $\bar{E}=\left(E,\left(\|\cdot\|_{v}\right)_{v}\right)$ sur Spec $k$ est la donnée d'un $k$-espace vectoriel $E$ de dimension finie $n$ et d'une famille de normes $\|.\|_{v}$ sur $E \otimes_{k} \mathbf{C}_{v}$, aux places $v$ de $k$, soumise aux contraintes suivantes:

1) Il existe une $k$-base $\left(e_{1}, \ldots, e_{n}\right)$ de $E$ telle que, pour toute place $v$ ultramétrique en dehors d'un nombre fini, la norme sur $E \otimes_{k} \mathbf{C}_{v}$ est donnée par

$$
\left\|\sum_{i=1}^{n} x_{i} e_{i}\right\|_{v}=\max _{1 \leq i \leq n}\left\{\left|x_{i}\right|_{v}\right\} .
$$

2) Soit $\operatorname{Gal}\left(\mathbf{C}_{v} \mid k_{v}\right)$ l'ensemble des automorphismes continus qui laissent invariants les éléments de $k_{v}$. Alors $\|\cdot\|_{v}$ est invariante sous l'action de $\operatorname{Gal}\left(\mathbf{C}_{v} \mid k_{v}\right)$ : étant donné une $k_{v}$-base $\left(\alpha_{1}, \ldots, \alpha_{n}\right)$ de $E \otimes_{k} k_{v}$ et $\left(x_{1}, \ldots, x_{n}\right) \in \mathbf{C}_{v}^{n}, \sigma \in \operatorname{Gal}\left(\mathbf{C}_{v} \mid k_{v}\right)$, on a

$$
\left\|\sigma\left(x_{1}\right) \alpha_{1}+\ldots+\sigma\left(x_{n}\right) \alpha_{n}\right\|_{v}=\left\|x_{1} \alpha_{1}+\cdots+x_{n} \alpha_{n}\right\|_{v} .
$$

3) Si $v$ est ultramétrique alors

$$
\forall x, y \in E \otimes_{k} \mathbf{C}_{v}, \quad\|x+y\|_{v} \leq \max \left\{\|x\|_{v},\|y\|_{v}\right\}
$$

(ultra-norme selon la terminologie de Bourbaki).

Un fibré en droites adélique est un fibré vectoriel adélique de dimension 1. Un fibré adélique hermitien est un fibré vectoriel adélique dont toutes les normes aux places archimédiennes de $k$ sont hermitiennes. Par extension, nous parlerons encore de fibré adélique hermitien lorsque $k$ est un corps de fonctions.

Cette définition appelle quelques commentaires. Tout d'abord, rappelons qu'un fibré vectoriel hermitien sur Spec $\mathcal{O}_{k}$ ( $k$ corps de nombres nécessairement) est la donnée d'un $\mathcal{O}_{k}$-module projectif de type fini $\mathcal{E}$ et de normes $\|\cdot\|_{v}$ euclidiennes (resp. hermitiennes) aux places archimédiennes réelles (resp. complexes) de $k$ sur les espaces $\mathcal{E} \otimes_{\mathcal{O}_{k}} k_{v}$. De plus, si $v$ est complexe, la norme $\|.\|_{v}$ est supposée invariante par conjugaison complexe. Cette hypothèse supplémentaire correspond exactement à la condition 2) ci-dessus. La cohérence avec la notion de fibré vectoriel adélique sur Spec $k$ est assurée grâce aux deux observations suivantes. D'une part, le module $\mathcal{E}$ fournit naturellement une structure entière de $E=\mathcal{E} \otimes k$ au sens où la norme en une place ultramétrique $v$ de $k$ que l'on choisit sur $E \otimes_{k} \mathbf{C}_{v}$ est donnée par

$$
\forall x \in E \otimes_{k} \mathbf{C}_{v}, \quad\|x\|_{E, v}:=\inf \left\{|a|_{v} ; a \in \mathbf{C}_{v}, x \in a \cdot\left(\mathcal{E} \otimes_{\mathcal{O}_{k}} \widehat{\mathcal{O}}_{v}\right)\right\}
$$


où l'anneau $\widehat{\mathcal{O}}_{v}$ est l'anneau de valuation de $\mathbf{C}_{v}$ (c.-à-d. sa boule unité fermée). En considérant une famille génératrice minimale de $\mathcal{E} \operatorname{sur} \mathcal{O}_{k}$, on vérifie que cette norme satisfait à la formule (13), et en particulier elle est invariante sous l'action de $\operatorname{Gal}\left(\mathbf{C}_{v} / k_{v}\right)$. D'autre part, une métrique euclidienne $\operatorname{sur} \mathcal{E} \otimes k_{v}$ ( $v$ réelle) se prolonge naturellement et de manière unique en une métrique hermitienne sur $\mathcal{E} \otimes \mathbf{C}_{v}$, car une telle métrique est déterminée par une matrice réelle symétrique définie positive, unique à conjugaison près par les éléments du groupe orthogonal $\mathrm{O}_{n}(\mathbf{R})$. Ce dernier point est remarquable. L'unicité du prolongement n'est plus vraie en général lorsque $\mathcal{E} \otimes k_{v}$ est muni d'une norme quelconque. Il n'existe pas de procédé «canonique» qui permette d'étendre une norme $\|$.$\| sur une espace vectoriel réel E \otimes \mathbf{R}$ au complexifié $E \otimes \mathbf{C} \simeq E \otimes \mathbf{R} \oplus i E \otimes \mathbf{R}$. Considérons par exemple un élément $p \in[1,+\infty]$ et posons, pour $a=a_{1}+i a_{2} \in E \otimes \mathbf{C}$,

$$
\|a\|_{p}^{\sim}:= \begin{cases}2^{\min \left\{\frac{1}{2}-\frac{1}{p}, 0\right\}}\left(\left\|a_{1}\right\|^{p}+\left\|a_{2}\right\|^{p}\right)^{1 / p} & \text { si } p<+\infty, \\ \max \left\{\left\|a_{1}\right\|,\left\|a_{2}\right\|\right\} & \text { si } p=+\infty .\end{cases}
$$

La fonction $a \mapsto\|a\|_{p}^{\sim}$ ne définit pas en général une norme car $\|\lambda a\|_{p}^{\sim}$ peut être différent de $|\lambda| \cdot\|a\|_{p}^{\sim}$ pour $\lambda \in \mathbf{C}$. En revanche, si l'on définit

$$
\|a\|_{p}^{\#}:=\sup \left\{\left\|e^{i \theta} a\right\|_{p}^{\sim} ; \theta \in[0,2 \pi]\right\},
$$

on vérifie alors que $\|.\|_{p}^{\#}$ est une norme sur $E \otimes \mathbf{C}$, invariante par conjugaison complexe et égale à $\|$.$\| sur E \otimes \mathbf{R}(*)$. Si $p=2$ et si $\|$.$\| est eu-$ clidienne alors $\|.\|_{2}^{\#}$ est la norme hermitienne qui prolonge $\|$.$\| à E \otimes \mathbf{C}$ (voir l'exemple 1.3 , p. 16, de [28]). Cette observation - absence d'un prolongement naturel - est la raison essentielle qui justifie que les normes associées à un fibré vectoriel adélique soient définies sur $E \otimes \mathbf{C}_{v}$ (ou $E \otimes \bar{k}_{v}$, ce qui revient au même quitte à prolonger ensuite par continuité, de manière unique, à $E \otimes \mathbf{C}_{v}$ ). Il est alors possible d'opérer une extension des scalaires en considérant une extension finie $K \mid k$ et le fibré vectoriel adélique $\bar{E}_{K}$ dont l'espace sous-jacent est $E \otimes K$, avec les mêmes normes que $\bar{E}$.

Par ailleurs, la première condition dans la définition 3.1, si elle est remplie, est alors vraie pour toute $k$-base de $E$, quitte éventuellement à accroître le nombre de places $v$ qui ne conviennent pas. En réalité, si l'on

(*) Cette dernière propriété est la raison pour laquelle le facteur de normalisation $2^{\min \left\{\frac{1}{2}-\frac{1}{p}, 0\right\}}$ apparaît dans la définition de $\|a\|_{p}^{\#}$, ce facteur étant égal à $\inf _{0 \leq \theta \leq 2 \pi}\left\{(\cos \theta)^{p}+(\sin \theta)^{p}\right\}^{-1 / p}$. 
fixe une $k$-base $\left(e_{1}, \ldots, e_{n}\right)$ de $E$, il existe une matrice d'adèles finis $\left(c_{v}\right)_{v} \in \mathrm{GL}_{n}\left(k_{\mathbf{A}, f}\right)$ telle que, pour toute place ultramétrique $v$ de $k$, on a

$$
\forall x \in \mathbf{C}_{v}^{n}, \quad\left\|x_{1} e_{1}+\cdots+x_{n} e_{n}\right\|_{v}=\max _{1 \leq i \leq n}\left\{\left|\left(c_{v} \cdot x\right)_{i}\right|_{v}\right\}
$$

où $\left(c_{v} \cdot x\right)_{i}$ désigne la $i$ ème coordonnée du vecteur $c_{v} \cdot x$. En effet, d'après la proposition 3 du chapitre II, p. 26, de [40], et étant donné une place ultramétrique $v$, il existe une $k_{v}$-base $\left(\alpha_{1}, \ldots, \alpha_{n}\right)$ de $E \otimes k_{v}$ telle que

$$
\forall x \in k_{v}^{n}, \quad\left\|x_{1} \alpha_{1}+\cdots+x_{n} \alpha_{n}\right\|_{v}=\max _{1 \leq i \leq n}\left\{\left|x_{i}\right|_{v}\right\}
$$

Autrement dit, il existe $c_{v} \in \mathrm{GL}_{n}\left(k_{v}\right)$ tel que la relation (15) soit satisfaite pour tout $x \in k_{v}^{n}$. La matrice $c_{v}$ est la matrice identité pour presque tout $v$. Pour obtenir l'égalité (15) avec $x \in \mathbf{C}_{v}^{n}$, on observe que si $K_{w}$ est une extension finie de $k_{v}$, il existe une matrice $c_{w} \in \mathrm{GL}_{n}\left(K_{w}\right)$ telle que

$$
\forall x \in K_{w}^{n}, \quad\left\|x_{1} e_{1}+\cdots+x_{n} e_{n}\right\|_{v}=\max _{1 \leq i \leq n}\left\{\left|\left(c_{w} \cdot x\right)_{i}\right|_{v}\right\} .
$$

En se restreignant à $x \in k_{v}^{n}$ et en choisissant les vecteurs de la base canonique de $k_{v}^{n}$, on constate que $c_{w} c_{v}^{-1} \in \mathrm{GL}_{n}\left(\mathcal{O}_{w}\right)\left(\mathcal{O}_{w}\right.$ est l'anneau de valuation de $K_{w}$ ) et l'égalité (17) reste vraie en remplaçant $c_{w}$ par $c_{v}$. Elle est donc valide pour $x \in \bar{k}_{v}^{n}$ et, par continuité, pour $x \in \mathbf{C}_{v}^{n}$.

$\mathrm{Si}$, comme nous l'avons vu plus haut, un fibré vectoriel hermitien $\overline{\mathcal{E}}$ sur Spec $\mathcal{O}_{k}$ fournit une structure entière pour $E=\mathcal{E} \otimes k$, la réciproque est également vraie. Si l'on se donne un fibré vectoriel adélique $\bar{E}$ sur Spec $k$, l'ensemble

$$
\mathcal{E}:=\left\{x \in E ; \forall v \nmid \infty,\|x\|_{E, v} \leq 1\right\}
$$

est une structure entière pour $E$. Dans cette écriture, si $k$ est un corps de fonctions, les places $v$ exclues sont celles qui sont au-dessus de la place $\infty$ de $\mathbf{F}_{p}[T]$ définie dans les préliminaires. Notons $\mathcal{O}_{k}:=\left\{x \in k ; \forall v \nmid \infty,|x|_{v} \leq 1\right\}$ l'anneau des entiers de $k$. Cet ensemble est aussi la fermeture intégrale de l'anneau

$$
\mathcal{O}_{k_{0}}:= \begin{cases}\mathbf{Z} & \text { si } k \text { est un corps de nombres } \\ \mathbf{F}_{p}[T] & \text { si } k \text { est un corps de fonctions }\end{cases}
$$

(voir [8], chap. VI, $\S 3$, corollaire 3), et à ce titre il est de Dedekind. L'ensemble $\mathcal{E}$ est un module sans torsion sur $\mathcal{O}_{k}$. Vu la caractérisation (15) des normes de $\bar{E}$ aux places ultramétriques, il existe un élément $N \in \mathcal{O}_{k_{0}} \backslash\{0\}$ tel que $N E \subseteq \mathcal{E}$, ce qui entraîne que $\mathcal{E}$ est de type 
fini. Cela montre que $\mathcal{E}$ est projectif de type fini (voir [20], chap. 1, § 9) et on vérifie que, pour toute place $v$ de $k$, qui ne domine pas la place $\infty$, la norme sur $E \otimes_{k} \mathbf{C}_{v}$ induite par $\mathcal{E}$ au moyen de la formule (14) est égale à la norme $\|\cdot\|_{E, v}$ de départ.

Enfin, signalons que certains auteurs accordent (ou accorderaient) aux normes $\|.\|_{v}$ de n'être que des semi-normes. Ici cela semble a priori exclu en partie à cause des résultats du paragraphe précédent dans les espaces normés dont nous aurons besoin.

\section{1. - Exemples de fibrés vectoriels adéliques.}

L'exemple le plus simple est celui de l'espace $k$ lui-même, qui, muni des différentes valeurs absolues $|\cdot|_{v}$, possède une structure de fibré vectoriel adélique (dite «triviale»). D'autres exemples sont les fibrés vectoriels adéliques que l'on va noter $\left(k^{n},|\cdot|_{p}\right)$ avec $p \in[1,+\infty]$, dont la structure entière est donnée par la base canonique de $k^{n}$ et où, en une place $v$ archimédienne, la norme $|\cdot|_{p}$ est celle définie par la formule (3). L'on pourrait bien sûr panacher plusieurs normes de ce type aux différentes places archimédiennes ou changer la structure entière en choisissant une autre base de $k^{n}$. Mais au-delà de ces exemples et à l'instar des fibrés vectoriels hermitiens sur $\operatorname{Spec} \mathcal{O}_{k}$, il existe d'autres exemples provenant de la géométrie algébrique, obtenus en considérant l'espace des sections globales d'un fibré en droites métrisé adéliquement sur une variété projective audessus de $k$ (voir $\S 8$ ).

\section{2. - Lien avec la notion de convexe adélique.}

Étant donné une place ultramétrique $v$ de $k$, une partie $C_{v}$ de $E \otimes k_{v}$ est appelé $k_{v}$-réseau si $C_{v}$ est un $\mathcal{O}_{v}$-sous-module de $E \otimes k_{v}$, à la fois ouvert et compact. Fixons une $k$-base de $E$ qui permet d'identifier $E$ à $k^{n}$. Dans toute la suite, nous supposons que le $k_{v}$-réseau $C_{v}$ s'identifie à $\mathcal{O}_{v}^{n}$, pour toute place $v$ en dehors d'un nombre fini. Si $v$ est une place archimédienne de $k$, soit $C_{v}$ un sous-ensemble convexe de $E \otimes k_{v}$, d'intérieur non vide, compact et symétrique par rapport à l'origine. L'origine est un point intérieur de $C_{v}$. Si $v$ est une place complexe, on suppose de plus que

$$
\forall \theta \in \mathbf{R}, \quad e^{i \theta} . C_{v}=C_{v} .
$$


DÉfinition 3.2. Un convexe adélique est un sous-ensemble $C$ de $E_{\mathbf{A}}=E \otimes k_{\mathbf{A}}$ de la forme $\prod_{v} C_{v}$, où les ensembles $C_{v}$ vérifient les hypothèses ci-dessus.

Si $\bar{E}$ est un fibré vectoriel adélique sur Spec $k$, la boule unité fermée de $\bar{E}$

$$
\mathbb{B}(\bar{E})=\left\{\left(x_{v}\right)_{v} \in E_{\mathbf{A}} ; \forall v,\left\|x_{v}\right\|_{v} \leq 1\right\}
$$

est un convexe adélique. En effet, l'ensemble $C_{v}=\left\{x_{v} \in E \otimes k_{v} ;\left\|x_{v}\right\|_{v} \leq 1\right\}$ convient dans tous les cas et la condition 1) de la définition 3.1 assure que $C_{v} \simeq \mathcal{O}_{v}^{n}$ pour presque tout $v$. On a également une réciproque :

Proposition 3.3. Tout convexe adélique sur $k$ est la boule unité fermée d'un fibré vectoriel adélique sur Speck, non nécessairement unique.

DÉmonstration. Si $v$ est une place archimédienne, l'on sait que la donnée d'un ensemble convexe $C_{v}$ comme ci-dessus équivaut à la donnée d'une norme sur $E \otimes k_{v}$ dont $C_{v}$ est la boule unité correspondante, norme donnée explicitement par l'application jauge $j_{v}: E \otimes k_{v} \rightarrow \mathbf{R}^{+}$:

$$
j_{v}(x)=\inf \left\{\lambda>0 ; \frac{x}{\lambda} \in C_{v}\right\} .
$$

La norme ainsi construite se prolonge à $E \otimes \mathbf{C}_{v}$ (sans que le prolongement ne soit unique). Comme nous l'avons déjà mentionné, l'hypothèse (18) intervient dans le cas complexe pour assurer l'égalité $j_{v}(a x)=|a| j_{v}(x)$ pour tous $a \in \mathbf{C}$ et $x \in E \otimes_{v} \mathbf{C}$.

Si $v$ est une place ultramétrique, soit $\pi_{v}$ une uniformisante de $\mathcal{O}_{v}$ (c.-à-d. un générateur de l'idéal maximal principal de $\mathcal{O}_{v}$ ). Et, comme dans le cas précédent, considérons pour $x \in E \otimes k_{v}$ le nombre réel

$$
j_{v}(x)=\inf \left\{\lambda=\left|\pi_{v}\right|_{v}^{h} ; h \in \mathbf{Z} \text { et } \frac{x}{\pi_{v}^{h}} \in C_{v}\right\} .
$$

Ce nombre est bien défini car $C_{v}$ est ouvert et la borne inférieure est un minimum car $C_{v}$ est compact. On vérifie alors que $j_{v}$ définit une norme ultramétrique sur $E \otimes k_{v}$ dont $C_{v}$ est la boule unité fermée. Comme nous l'avons vu plus haut, cette norme s'étend à $\mathbf{C}_{v}^{n}$ via le choix d'une matrice $c_{v} \in \mathrm{GL}_{n}\left(k_{v}\right)$ convenable. L'hypothèse $C_{v} \simeq \mathcal{O}_{v}^{n}$ permet de prendre $c_{v}$ égal à la matrice identité pour presque tout $v$. La collection des normes $\left(j_{v}\right)_{v}$, prolongées aux espaces $E \otimes \mathbf{C}_{v}$, fournit la structure adélique recherchée. 
REMARque 3.4. Si $E$ est une droite, il n'y a plus d'ambiguïté selon que l'on regarde $E \otimes k_{v}$ ou $E \otimes \mathbf{C}_{v}$ ( $v$ archimédienne) ; les métriques aux éventuelles places archimédiennes de $k$ sont alors automatiquement hermitiennes.

Il arrive souvent que les normes aux places ultramétriques proviennent toutes d'une même base $\left(e_{1}, \ldots, e_{n}\right)$ de $E$, comme dans la formule (13) de la définition 3.1. Cette base fournit la structure entière du fibré vectoriel adélique $\bar{E}$. Lorsque $E=k^{n}$, la base canonique donne une structure entière naturelle, utilisée par défaut dans la suite.

\section{3. - Opérations algébriques sur l'ensemble des fibrés vectoriels adéliques.}

Soit $\bar{E}, \bar{F}$ des fibrés vectoriels adéliques sur Spec $k$ de dimensions respectives $n$ et $m$.

Sous-fibré et quotient.

Nous dirons que $\bar{F}$ est un sous-fibré de $\bar{E}$, et nous écrirons $\bar{F} \subseteq \bar{E}$, si $F \subseteq E$, et si, en chaque place $v$, la norme $\|\cdot\|_{F, v}$ est la restriction de $\|\cdot\|_{E, v}$ à $F \otimes_{k} \mathbf{C}_{v}$. De même, si $\bar{F} \subseteq \bar{E}$ alors le quotient $E / F$ est muni d'une structure de fibré vectoriel adélique sur Speck en considérant les normes quotient.

\section{Somme directe.}

Il n'existe pas de norme plus naturelle qu'une autre sur la somme directe (ou le produit) d'espaces vectoriels normés. Afin d'assurer la compatibilité avec la somme directe hermitienne de fibrés vectoriels hermitiens, la norme, par défaut, que nous choisirons sur $E \oplus F$ est, pour tous $x \in E \otimes \mathbf{C}_{v}, y \in F \otimes \mathbf{C}_{v}$,

$$
\|(x, y)\|_{E \oplus F, v}:= \begin{cases}\max \left\{\|x\|_{E, v},\|y\|_{F, v}\right\} & \text { si } v \text { est ultramétrique, } \\ \left(\|x\|_{E, v}^{2}+\|y\|_{F, v}^{2}\right)^{1 / 2} & \text { si } v \text { est archimédienne. }\end{cases}
$$

Nous noterons $\bar{E} \oplus_{2} \bar{F}$ ou, plus simplement, $\bar{E} \oplus \bar{F}$, le fibré vectoriel adélique obtenu de la sorte. Si l'on remplace la norme $|\cdot|_{2}$ par la norme $|\cdot|_{p}$, $p \in[1,+\infty]$, nous noterons $\bar{E} \oplus_{p} \bar{F}$ le fibré vectoriel adélique que l'on obtient. Il peut être utile parfois d'avoir (encore) un peu plus de souplesse dans 
le choix des normes. Étant donné une place archimédienne $v$ de $k$, considérons $\varsigma_{v}$ une norme symétrique sur $\mathbf{R}^{2}$, invariante par changement de signes sur les coordonnées, telle que $\varsigma_{v}(1,0)=\varsigma_{v}(0,1)=1$. Posons $\varsigma:=\left(\varsigma_{v}\right)_{v \mid \infty}$. Le fibré vectoriel adélique $\bar{E} \oplus_{\varsigma} \bar{F}$ est par définition l'espace $E \oplus F$ muni, aux places archimédiennes, des normes $\|(x, y)\|_{E \oplus_{c} F, v}:=\varsigma_{v}\left(\|x\|_{E, v},\|y\|_{F, v}\right)$. Les normes aux places ultramétriques sont les mêmes que précédemment.

REMARque 3.5. Ces opérations préservent la structure hermitienne quand les objets de départ en sont pourvus.

\section{Dual et norme d'opérateur.}

Le fibré vectoriel dual $\bar{E}^{\vee}$ est l'espace dual $E^{\vee}=\operatorname{Hom}_{k}(E, k)$, muni des normes duales usuelles:

$$
\forall \varphi \in\left(E \otimes \mathbf{C}_{v}\right)^{v}, \quad\|\varphi\|_{E^{v}, v}:=\sup \left\{\frac{|\varphi(x)|_{v}}{\|x\|_{E, v}} ; x \in E \otimes \mathbf{C}_{v}, x \neq 0\right\} .
$$

Plus généralement, l'espace $\operatorname{Hom}_{k}(E, F)$ des applications linéaires entre $E$ et $F$ est muni aux différentes places $v$ des normes d'opérateurs usuelles, obtenues en remplaçant $|\varphi(x)|_{v}$ par $\|\varphi(x)\|_{F, v}$ dans l'expression ci-dessus. D'une manière alternative, on a, pour tout $\varphi \in \operatorname{Hom}_{k}(E, F) \otimes \mathbf{C}_{v}$,

$$
\|\varphi\|_{\operatorname{Hom}(E, F), v}=\sup \left\{y^{\mathrm{v}}(\varphi(x)) ;\left\|y^{\mathrm{v}}\right\|_{F^{\mathrm{v}}, v}=\|x\|_{E, v}=1\right\} .
$$

REMARque 3.6. Le bidual $\left(\overline{E^{v}}\right)^{v}$ est isomorphe isométriquement à $\bar{E}$ et l'on dispose de la formule (vraie pour toute place $v$ de $k$ )

$$
\forall x \in E \otimes_{k} \mathbf{C}_{v}, \quad\|x\|_{E, v}=\sup _{\|\varphi\|_{E^{v}, v} \leq 1}|\varphi(x)|_{v} .
$$

Dans le cas archimédien, il s'agit d'un résultat classique de la théorie des espaces vectoriels normés de dimension finie (voir [9], chap. IV, § 2.4). Dans le cas ultramétrique, l'égalité (19) peut se démontrer au moyen de la base $\left(\alpha_{1}, \ldots, \alpha_{n}\right)$ de $E \otimes \mathbf{C}_{v}$ donnant la formule (16).

\section{Produit tensoriel.}

La norme d'opérateur confère au produit tensoriel $E \otimes_{k} F$ une structure de fibré vectoriel adélique via l'isomorphisme $E \otimes_{k} F \simeq \operatorname{Hom}_{k}\left(E^{\vee}, F\right)$. Malheureusement, la norme tensorielle ainsi bâtie sur $E \otimes_{k} F$ ne donne pas en général une structure hermitienne à $E \otimes_{k} F$ lorsque $\bar{E}$ et $\bar{F}$ sont des 
fibrés vectoriels hermitiens (sauf si $E$ ou $F$ est une droite, ou si $k$ est un corps de fonctions).

DÉfinition 3.7. Soit $\ell \in \mathbf{N} \backslash\{0\}$. Une norme tensorielle adélique d'ordre $\ell$ sur $k$ (corps global) est la donnée d'une famille $\alpha=\left(\alpha_{v}\right)_{v}$ indexée par les places $v$ de $k$ telle que :

1) Si $v$ est archimédienne, $\alpha_{v}$ est une norme tensorielle d'ordre $\ell$ (au sens de la définition 2.10).

2) Si $v$ est ultramétrique alors $\alpha_{v}$ est la norme tensorielle ultramétrique définie de la manière suivante : pour tout $i \in\{1, \ldots, \ell\}$, pour tout $\mathbf{C}_{v}$-espace vectoriel ultra-normé $\left(E_{i},\|.\|_{E_{i}}\right)$ de dimension finie $n_{i}$, la norme $\alpha_{v}\left(\cdot ; E_{1}, \ldots, E_{\ell}\right) \operatorname{sur} \otimes_{i=1}^{\ell} E_{i}$ est l'ultra-norme définie par récurrence sur $\ell$ : $\alpha_{v}\left(\cdot ; E_{1}\right)=\|\cdot\|_{E_{1}}$ et $\alpha_{v}\left(\cdot ; E_{1}, \ldots, E_{\ell}\right)$ s'identifie à la norme d'opérateur des applications linéaires entre l'espace normé dual $\left(E_{1}^{\mathrm{v}},\|\cdot\|_{E_{1}^{\mathrm{v}}}\right)$ et l'espace normé $\left(E_{2} \otimes \cdots \otimes E_{\ell}, \alpha_{v}\left(\cdot ; E_{2}, \ldots, E_{\ell}\right)\right)$.

Une norme tensorielle (adélique) hermitienne est la donnée d'une norme tensorielle adélique $\alpha=\left(\alpha_{v}\right)_{v}$ telle que, pour toute place archimédienne $v$ de $k$, la norme tensorielle $\alpha_{v}$ est hermitienne au sens de la définition 2.10.

Étant donné des fibrés vectoriels adéliques $\bar{E}_{1}, \ldots, \bar{E}_{\ell}$ sur Spec $k$, on notera $\alpha\left(\cdot ; \bar{E}_{1}, \ldots, \bar{E}_{\ell}\right)$ la collection des normes $\alpha_{v}\left(\cdot ;\left(E_{1} \otimes_{k} \mathbf{C}_{v},\|\cdot\|_{\bar{E}_{1, v}}\right), \ldots,\left(E_{\ell} \otimes_{k} \mathbf{C}_{v},\|\cdot\|_{\bar{E}_{\ell, v}}\right)\right)$ sur $E_{1} \otimes_{k} \cdots \otimes_{k} E_{\ell} \otimes_{k} \mathbf{C}_{v}$, lorsque $v$ varie parmi les places de $k$. La définition 3.7 assure que si $\alpha$ est une norme tensorielle adélique, alors le couple

$$
\otimes_{\alpha, i=1}^{\ell} \bar{E}_{i}:=\left(E_{1} \otimes_{k} \cdots \otimes_{k} E_{\ell}, \alpha\left(\cdot ; \bar{E}_{1}, \ldots, \bar{E}_{\ell}\right)\right)
$$

est un fibré vectoriel adélique sur Spec $k$ (lorsque tous les $\bar{E}_{i}$ sont égaux à $\bar{E}$, on notera aussi $\left.\bar{E}^{\otimes_{\alpha} \ell}:=\otimes_{\alpha, i=1}^{\ell} \bar{E}\right)$. Et, de plus, si tous les $\bar{E}_{i}$ et $\alpha$ sont hermitiens alors $\otimes_{\alpha, i=1}^{\ell} \bar{E}_{i}$ est un fibré adélique hermitien. En choisissant aux places archimédiennes de $k$ les normes de Chevet-Saphard définies dans l'exemple 2.11, on obtient des exemples de normes tensorielles adéliques d'ordre 2, que l'on peut ensuite étendre à tout ordre. En particulier si $\alpha_{v}=g_{2}$ pour toute place $v$ archimédienne alors la norme tensorielle adélique $\left(\alpha_{v}\right)_{v}$, que nous noterons encore $g_{2}$, est hermitienne.

Remarque 3.8. Par récurrence sur $\ell$, la formule de bidualité (19) fournit une expression concrète de la norme d'opérateur généralisée du point 2) de la définition 3.7, sous la forme suivante: pour tout entier $h \geq 1$, 
pour tous $x_{j}^{(i)} \in E_{j}, 1 \leq j \leq \ell$ et $1 \leq i \leq h$, on a

$$
\begin{aligned}
& \alpha_{v}\left(\sum_{i=1}^{h} \otimes_{j=1}^{\ell} x_{j}^{(i)} ; E_{1}, \ldots, E_{\ell}\right) \\
& \quad=\sup \left\{\left|\sum_{i=1}^{h} \prod_{j=1}^{\ell} \varphi_{j}\left(x_{j}^{(i)}\right)\right|_{v} ; \forall j \in\{1, \ldots, \ell\},\left\|\varphi_{j}\right\|_{E_{j}^{\vee}} \leq 1\right\} .
\end{aligned}
$$

Produit extérieur et déterminant.

L'algèbre extérieure $\bigwedge(E)$ est le quotient de l'algèbre tensorielle $\mathbf{T}(E)$ par l'idéal bilatère engendré par les éléments $x \otimes x$ avec $x \in E$. Soit $\ell \in\{1, \ldots, n\}$. Le $\ell^{\text {ème }}$ produit extérieur $\bigwedge^{\ell}(E)$ est donc un quotient du produit tensoriel $E^{\otimes \ell}$. Il hérite alors de la structure adélique quotient induite par celle définie précédemment sur $\bar{E}^{\otimes_{\alpha} \ell}$ au moyen d'une norme tensorielle adélique $\alpha$ d'ordre $\ell$. En particulier, si $\ell=n$, le déterminant $\operatorname{det}(E)=\bigwedge^{n}(E)$ de $E$ possèdent une structure de fibré vectoriel adélique sur Spec $k$. Nous noterons $\bigwedge_{\alpha}^{\ell}(\bar{E})$ et $\operatorname{det}_{\alpha} \bar{E}$ les fibrés adéliques que l'on obtient ainsi. Si $\bar{E}$ et $\alpha$ sont hermitiens alors le fibré vectoriel adélique $\bigwedge_{\alpha}^{\ell}(\bar{E})$ est également hermitien et l'on a dans ce cas, pour toute place archimédienne $v$ de $k$,

$$
\left\|e_{1} \wedge \cdots \wedge e_{\ell}\right\|_{\wedge_{\alpha}^{\ell}(E), v}=\operatorname{det}\left(\left(e_{i}, e_{j}\right)_{v}\right)_{i, j}^{1 / 2} \quad \text { pour tous } e_{1}, \ldots, e_{\ell} \in E \otimes \mathbf{C}_{v}
$$

$\left((,)_{v}\right.$ désigne le produit hermitien sur $\left.E \otimes \mathbf{C}_{v}\right)$.

\section{Produit symétrique.}

L'algèbre symétrique $\mathbf{S}(E)$ est le quotient de l'algèbre tensorielle $\mathbf{T}(E)$ par l'idéal bilatère engendré par les éléments de la forme $x \otimes y-y \otimes x$. Pour $\ell \in \mathbf{N}$, la structure de fibré vectoriel adélique sur la puissance symétrique $S^{\ell}(E)$ est celle obtenue par quotient de celle de $\bar{E}^{\otimes_{\alpha} \ell}$, une norme tensorielle adélique $\alpha$ d'ordre $\ell$ ayant été fixée au préalable. Nous noterons $S_{\alpha}^{\ell}(\bar{E})$ le fibré vectoriel adélique obtenu de la sorte. Si $\bar{E}$ et $\alpha$ sont hermitiens alors $S_{\alpha}^{\ell}(\bar{E})$ est également hermitien. Dans ce cas l'on peut préciser la valeur de la norme en une place archimédienne $v$ de $k$ d'un élément de $S^{\ell}(E) \otimes_{v} \mathbf{C}$ de la manière suivante. Soit $\sigma_{E, v}: E^{\otimes \ell} \otimes_{v} \mathbf{C} \rightarrow E^{\otimes \ell} \otimes_{v} \mathbf{C}$ l'opérateur de symétrisation: pour tous $x_{1}, \ldots, x_{\ell} \in E \otimes_{v} \mathbf{C}$, on a $\sigma_{E, v}\left(x_{1} \otimes \cdots \otimes x_{\ell}\right)=\frac{1}{\ell !} \sum_{\eta \in \Xi_{\ell}} x_{\eta(1)} \otimes \cdots \otimes x_{\eta(\ell)}$ ( $\Im_{\ell}$ est le groupe symétrique d'ordre $\ell$ ). L'image de $\sigma_{E, v}$, munie de la norme restreinte de $E^{\otimes \ell} \otimes_{v} \mathbf{C}$, est un espace hermitien, isomorphe iso- 
métriquement à $S^{\ell}(E) \otimes_{v} \mathbf{C}$, muni de la norme quotient comme ci-dessus. De la sorte, si $e_{1}, \ldots, e_{n}$ désigne une base orthonormée de $E \otimes_{v} \mathbf{C}$, la famille $\left\{e_{1}^{i_{1}} \cdots e_{n}^{i_{n}} ;\left(i_{1}, \ldots, i_{n}\right) \in \mathbf{N}^{n}\right.$ et $\left.i_{1}+\cdots+i_{n}=\ell\right\}$ forme une base orthogonale de $S^{\ell}(E) \otimes_{v}$ C. Les normes des vecteurs de cette base se calculent au moyen de la définition de $\sigma_{E, v}$ et l'on obtient

$$
\left\|e_{1}^{i_{1}} \cdots e_{n}^{i_{n}}\right\|_{S^{\ell}(E) \otimes_{v} \mathbf{C}}=\left\|\sigma_{E, v}\left(e_{1}^{\otimes i_{1}} \otimes \cdots \otimes e_{n}^{\otimes i_{n}}\right)\right\|_{E^{\otimes \ell} \bigotimes_{v} \mathbf{C}}=\left(\frac{i_{1} ! \cdots i_{n} !}{\ell !}\right)^{1 / 2}
$$

(voir [9], § 3.3 du cinquième chapitre).

REMARques 3.9.

- Il existe d'autres normes adéliques «naturelles» sur le produit symétrique qui ne sont pas obtenues par quotient. Toutefois, une telle norme «naturelle» se compare à une norme obtenue par quotient d'une norme tensorielle, avec des constantes de comparaison ne faisant intervenir que $\ell$ (voir [15]).

- Lorsque $\bar{E}$ et $\alpha$ sont hermitiens, les fibrés adéliques $\bar{E}^{\otimes_{\alpha} \ell}, \bigwedge_{\alpha}^{\ell}(\bar{E})$ et $S_{\alpha}^{\ell}(\bar{E})$ ne dépendent pas de $\alpha$ et nous simplifierons la notation en ne mettant pas l'indice $\alpha$.

\section{Notion de degré adélique et propriétés.}

Soit $\bar{E}=\left(E,\left(\|\cdot\|_{v}\right)_{v}\right)$ un fibré vectoriel adélique sur Spec $k$, de dimension $n \geq 1$. La boule unité de $\bar{E}$ est l'ensemble

$$
\mathbb{B}(\bar{E}):=\left\{\left(x_{v}\right)_{v} \in E_{\mathbf{A}} ; \quad \forall v \text { place de } k, \quad\left\|x_{v}\right\|_{v} \leq 1\right\} .
$$

DÉFINITION 4.1. Soit $\phi: E \rightarrow k^{n}$ un isomorphisme d'espaces vectoriels. Soit vol une mesure de Haar sur $k_{\mathrm{A}}^{n}$. Le degré adélique de $\bar{E}$ est le nombre réel:

$$
\widehat{\operatorname{deg}} \bar{E}:=\log \frac{\operatorname{vol}(\phi(\mathbb{B}(\bar{E})))}{\operatorname{vol}\left(\mathbb{B}\left(k^{n},|\cdot|_{2}\right)\right)} .
$$

Le degré adélique normalisé est

$$
\widehat{\operatorname{deg}}_{n} \bar{E}:=\frac{\widehat{\operatorname{deg}} \bar{E}}{\left[k: k_{0}\right]} .
$$

Ce nombre est indépendant des choix de l'isomorphisme $\phi$, en raison de la formule du produit, et de la mesure de Haar vol, car elles sont toutes pro- 
portionnelles. Par convention, si $E=\{0\}$, on pose $\widehat{\operatorname{deg}} \bar{E}:=0$. Pour la suite, il est utile de mentionner dès à présent le comportement de ce degré par changement d'échelle. Soit $A=\left(A_{v}\right)_{v} \in \mathrm{GL}\left(E_{\mathrm{A}}\right)$. On note $A \cdot \bar{E}=(E, A .\|\|$. le fibré vectoriel adélique dont l'espace sous-jacent est $E$ et dont la norme en une place $v$ de $k$ est donnée par $\|x\|_{v}:=\left\|A_{v}(x)\right\|_{E, v}$. Le déterminant de $A$ calculé dans une $k$-base de $E$ s'identifie à un idèle de $k_{\mathrm{A}}$ dont la valeur absolue $|\operatorname{det} A|_{\mathrm{A}}$ ne dépend pas du choix de cette $k$-base. Compte tenu des normalisations du début de ce texte (en particulier la formule (2)), on déduit la relation

$$
\widehat{\operatorname{deg}}(E, A .\|.\|)=\widehat{\operatorname{deg}} \bar{E}-\log |\operatorname{det} A|_{\mathbf{A}} .
$$

Par ailleurs et bien que nous ne l'utiliserons pas dans la suite, signalons qu'il existe également une caractéristique d'Euler-Poincaré d'un fibré vectoriel adélique $\bar{E}$, que l'on peut définir en posant

$$
\chi(\bar{E}):=\log \frac{\operatorname{vol}(\mathbb{B}(\bar{E}))}{\operatorname{covol}(E)}
$$

où vol est une mesure de Haar quelconque $\operatorname{sur} E_{\mathrm{A}}$ et $\operatorname{covol}(E)=\operatorname{vol}\left(E_{\mathrm{A}} / E\right)$. Le lien avec le degré adélique s'exprime au travers d'une formule analogue à celle de Riemann-Roch :

$$
\chi(\bar{E})=\widehat{\operatorname{deg}} \bar{E}+\chi\left(k^{n},|\cdot|_{2}\right)
$$

(voir [34, 36]).

\section{Notation.}

Soit $\overline{E_{1}}=\left(E,\|\cdot\|_{1}\right)$ et $\overline{E_{2}}=\left(E,\|\cdot\|_{2}\right)$ deux fibrés vectoriels adéliques ayant le même espace sous-jacent $E$. Si, en toute place $v$ de $k$ et pour tout $x \in E \otimes_{k} \mathbf{C}_{v}$, on a $\|x\|_{1, v} \leq\|x\|_{2, v}$ alors nous écrirons $\overline{E_{1}} \preceq \overline{E_{2}}$.

L'intérêt de cette écriture est le fait suivant.

LEMme 4.2. Si $\overline{E_{1}} \preceq \overline{E_{2}}$ alors $\widehat{\operatorname{deg}} \overline{E_{2}} \leq \widehat{\operatorname{deg}} \overline{E_{1}}$.

Cela résulte de l'inclusion des boules unités $\mathbb{B}\left(\overline{E_{2}}\right) \subseteq \mathbb{B}\left(\overline{E_{1}}\right)$.

Lemme 4.3. Soit $\alpha$ une norme tensorielle adélique d'ordre $\ell \in \mathbf{N} \backslash\{0\}$. Pour tout $i \in\{1, \ldots, \ell\}$, soit $\bar{E}_{i} \preceq \bar{F}_{i}$ deux fibrés vectoriels adéliques sur Speck. Alors $\otimes_{\alpha, i=1}^{\ell} \bar{E}_{i} \preceq \otimes_{\alpha, i=1}^{\ell} \bar{F}_{i}$. En particulier on a $\bar{E}_{1}^{\otimes_{\alpha} \ell} \preceq \bar{F}_{1}^{\otimes_{\alpha} \ell}$, $\bigwedge_{\alpha}^{\ell} \bar{E}_{1} \preceq \bigwedge_{\alpha}^{\ell} \bar{F}_{1}$, $\operatorname{det}_{\alpha} \bar{E}_{1} \preceq \operatorname{det}_{\alpha} \bar{F}_{1}\left(\right.$ si $\left.\ell=\operatorname{dim} E_{1}\right)$ et $S_{\alpha}^{\ell}\left(\bar{E}_{1}\right) \preceq S_{\alpha}^{\ell}\left(\bar{F}_{1}\right)$. 
Le premier résultat découle directement de la proposition 2.12. Et les suivants sont conséquences des définitions.

Ces observations seront souvent utilisées dans la suite.

\section{1. - Exemples.}

Au paragraphe 3.1, nous avons introduit le fibré vectoriel adélique $\left(k^{n},|\cdot|_{p}\right), p \in[1,+\infty]$. Si $k$ est un corps de fonctions alors le degré adélique de $\left(k^{n},|\cdot|_{p}\right)$ est nul. Si $k$ est un corps de nombres, les calculs (4) et (5) des volumes des boules unités réelle et complexe donnent la formule exacte :

$$
\widehat{\operatorname{deg}}\left(k^{n},|\cdot|_{p}\right)=r_{1} \log \frac{\Gamma\left(1+\frac{1}{p}\right)^{n} \Gamma\left(1+\frac{n}{2}\right)}{\Gamma\left(1+\frac{n}{p}\right)(\sqrt{\pi})^{n}}+r_{2} \log \frac{\Gamma\left(1+\frac{2}{p}\right)^{n} n !}{\Gamma\left(1+\frac{2 n}{p}\right)},
$$

où $r_{1}$ et $r_{2}$ sont respectivement le nombre de places réelles et complexes de $k$. Lorsque $n \longrightarrow+\infty$ et $p$ est fixé, la formule de Stirling, qui se trouve en note au bas de la page 29 , fournit le développement asymptotique

$$
\widehat{\operatorname{deg}}\left(k^{n},|\cdot|_{p}\right)=\operatorname{an} \log (n)+b n+c+o(1)
$$

avec

$$
\begin{aligned}
a & :=\left(\frac{1}{2}-\frac{1}{p}\right)[k: \mathbf{Q}], \\
b & :=r_{1} \log \frac{\Gamma\left(1+\frac{1}{p}\right)(p e)^{1 / p}}{\sqrt{2 e \pi}}+r_{2} \log \frac{\Gamma\left(1+\frac{2}{p}\right)(p / 2)^{2 / p}}{e^{1-2 / p}} \\
c & :=\frac{\left(r_{1}+r_{2}\right)}{2} \log \left(\frac{p}{2}\right) .
\end{aligned}
$$

4.2. - Expressions alternatives du degré adélique.

Lemme 4.4. Soit $\bar{E}$ un fibré en droites adélique et $s \in E \backslash\{0\}$. On a

$$
\widehat{\operatorname{deg}} \bar{E}=-\sum_{v} n_{v} \log \|s\|_{v} .
$$

DÉmonstration. Il suffit d'observer que si $x=\lambda . s \in E \otimes k_{v}, \lambda \in k_{v}$, alors $\|x\|_{v} \leq 1$ équivaut à $|\lambda|_{v} \leq \frac{1}{\|s\|_{v}}$. L'égalité (2) fait le lien entre les volumes et permet de conclure. 
On en déduit le

LEMme 4.5. Pour tout fibré adélique hermitien $\bar{E}$, on $a \widehat{\operatorname{deg}} \bar{E}=$ $=\widehat{\operatorname{deg}} \operatorname{det} \bar{E}$.

DÉmonstration. Nous avons vu que les normes de $\bar{E}$ aux places ultramétriques peuvent s'exprimer au moyen d'un adèle fini $\left(c_{v}\right)_{v \nmid \infty} \in$ $\in \mathrm{GL}_{n}\left(k_{\mathbf{A}, f}\right)$, après le choix d'une $k$-base $\mathbf{e}:=\left(e_{1}, \ldots, e_{n}\right)$ de $E$. L'hypothèse faite ici sur $\bar{E}$ signifie que le même phénomène se produit aux places archimédiennes et qu'il existe un prolongement $c=\left(c_{v}\right) \in$ $\in \mathrm{GL}_{n}\left(k_{\mathrm{A}}\right)$ tel que, pour toute place $v$ de $k$, on a

$$
\left(E_{v} \simeq_{\mathbf{e}} k_{v}^{n},\|\cdot\|_{v}\right)=c_{v} \cdot\left(k_{v}^{n},|\cdot|_{2}\right) .
$$

Ainsi chacun des quotients

$$
\frac{\operatorname{vol}\left(\left\{x \in k_{v}^{n} ;\|x\|_{v} \leq 1\right\}\right)}{\operatorname{vol}\left(\left\{x \in k_{v}^{n} ;|x|_{2, v} \leq 1\right\}\right)}
$$

égale $\left|\operatorname{det}\left(c_{v}\right)\right|_{v}^{-n_{v}}=\left\|e_{1} \wedge \cdots \wedge e_{n}\right\|_{v}^{-n_{v}}$. Le lemme précédent permet de conclure.

Ces deux lemmes montrent que le degré adélique coïncide avec le degré d'Arakelov sur le domaine de définition de ce dernier, à savoir les fibrés vectoriels hermitiens sur $\operatorname{Spec} \mathcal{O}_{k}$. Le degré adélique en est donc une généralisation.

Lorsque $k$ possède des places archimédiennes, la norme $\|\cdot\|_{v}$ de $\bar{E}$ en une telle place est comparable à la norme de John, introduite à la suite de la définition 2.4. Notons $|\cdot|_{J(\bar{E}), v}$ cette norme hermitienne étendue à l'espace $E \otimes \mathbf{C}_{v}$.

DÉfinition 4.6. Avec les données ci-dessus, nous appelerons fibré vectoriel adélique de John, ou plus simplement fibré de John, associé à $\bar{E}$, et nous noterons $J(\bar{E})$, le fibré vectoriel adélique obtenu à partir de $\bar{E}$ en remplaçant, aux places archimédiennes $v$ de $k$, la norme $\|\cdot\|_{v}$ par la norme hermitienne $|\cdot|_{J(\bar{E}), v^{*}}$

Le fibré de John est donc égal à $\bar{E}$ lorsque ce dernier est un fibré adélique hermitien. De la même manière, on définit le fibré vectoriel adélique de Löwner associé à $\bar{E}$ - que l'on note $L(\bar{E})$ - au moyen des métriques $|\cdot|_{L\left(\mathbf{B}\left(E_{v},\|\cdot\|_{v}\right)\right.}$ introduites au paragraphe 2.2, en une place ar- 
chimédienne $v$ de $k$. On notera que si $E^{\prime}$ est un sous-espace vectoriel de $E$ alors $\left(E^{\prime},|\cdot|_{L(\bar{E})}\right) \preceq\left(E^{\prime},\|\cdot\|_{E}\right) \preceq\left(E^{\prime},|\cdot|_{J(\bar{E})}\right)$ et donc, par le lemme 4.2, on a

$$
\widehat{\operatorname{deg}}\left(E^{\prime},|\cdot|_{J(\bar{E})}\right) \leq \widehat{\operatorname{deg}}\left(E^{\prime},\|\cdot\|_{E}\right) \leq \widehat{\operatorname{deg}}\left(E^{\prime},|\cdot|_{L(\bar{E})}\right) .
$$

On peut même intercaler $\widehat{\operatorname{deg}} J\left(\overline{E^{\prime}}\right)$ entre $\widehat{\operatorname{deg}}\left(E^{\prime},|\cdot|_{J \bar{E})}\right)$ et $\widehat{\operatorname{deg}}\left(E^{\prime},\|\cdot\|_{E}\right)$ en utilisant la définition des métriques de $J\left(\overline{E^{\prime}}\right)$ qui sont les métriques euclidiennes qui optimisent par valeur inférieure le volume de la boule unité de $\overline{E^{\prime}}$. La même observation avec $L\left(\overline{E^{\prime}}\right)$ conduit à

$$
\widehat{\operatorname{deg}}\left(E^{\prime},|\cdot|_{J(\bar{E})}\right) \leq \widehat{\operatorname{deg}} J\left(\overline{E^{\prime}}\right) \leq \widehat{\operatorname{deg}}\left(E^{\prime},\|\cdot\|_{E}\right) \leq \widehat{\operatorname{deg}} L\left(\overline{E^{\prime}}\right) \leq \widehat{\operatorname{deg}}\left(E^{\prime},|\cdot|_{L(\bar{E})}\right)
$$

Le fibré de John permet de donner une formule exacte pour le degré adélique de $\bar{E}$ grâce à la notion de quotient volumique (voir définition 2.5).

DÉFINITION 4.7. Soit $\bar{E}$ un fibré vectoriel adélique sur Spec $k$. Le quotient volumique adélique de $\bar{E}$, noté vr $(\bar{E})$, est la norme de l'adèle de composantes $\operatorname{vr}\left(E \otimes_{k} k_{v}\right)$ - le quotient volumique de l'espace de Banach réel ou complexe $\left(E \otimes_{k} k_{v},\|\cdot\|_{E, v}\right)$ - aux places $v$ archimédiennes et 1 aux autres places :

$$
\operatorname{vr}(\bar{E}):=\prod_{v \text { archimédienne }} \operatorname{vr}\left(E \otimes_{k} k_{v}\right)^{n_{v}} .
$$

Si $k$ est un corps de fonctions, on a donc toujours vr $(\bar{E})=1$. Ce nombre est un terme d'erreur qui intervient souvent lors de comparaisons entre la situation adélique générale et le cas hermitien. On a $\operatorname{vr}(\bar{E})=1$ si et seulement si $\bar{E}$ est un fibré adélique hermitien. Aussi, les inégalités établies dans la suite et qui font intervenir cette quantité sont des égalités dans le cas hermitien. De manière ponctuelle, nous aurons besoin également du nombre réel

$$
\widetilde{\operatorname{vr}}(\bar{E}):=\prod_{v \text { archimédienne }} \tilde{\operatorname{vr}}\left(E \otimes_{k} k_{v}\right)^{n_{v}}
$$

(voir définition 2.6 pour la notation $\widetilde{v}$ ). Introduisons maintenant l'analogue adélique de la distance de Banach-Mazur classique (définition 2.7).

DÉfinition 4.8. Soit $\bar{E}$ et $\bar{F}$ deux fibrés vectoriels adéliques sur $\operatorname{Spec} k$ de même dimension. La distance de Banach-Mazur adélique entre $\bar{E}$ et $\bar{F}$ est le nombre réel

$$
\mathrm{d}(\bar{E}, \bar{F}):=\prod_{v \text { archimédienne }} \mathrm{d}\left(E \otimes_{k} k_{v}, F \otimes_{k} k_{v}\right)^{n_{v}} .
$$

Si $k$ est un corps de fonctions, on pose $\mathrm{d}(\bar{E}, \bar{F})=1$. 
Notation. Lorsque $\bar{F}=\left(k^{n},|\cdot|_{2}\right)$, on notera plus simplement $\Delta(\bar{E})$ la distance $\mathrm{d}\left(\bar{E},\left(k^{n},|\cdot|_{2}\right)\right)$.

On montre que si $\overline{E^{\prime}}$ est un sous-fibré vectoriel adélique de $\bar{E}$ alors $\Delta\left(\overline{E^{\prime}}\right) \leq \Delta(\bar{E})$. De plus, le dual $\overline{E^{v}}$ de $\bar{E}$ vérifie $\Delta\left(\overline{E^{v}}\right)=\Delta(\bar{E})$. Grâce aux estimations locales (7) et (12), on dispose de l'encadrement

$$
1 \leq \operatorname{vr}(\bar{E}) \operatorname{vr}\left(\overline{E^{\vee}}\right) \leq \Delta(\bar{E}) \leq(2 n)^{D / 2} .
$$

Proposition 4.9. Soit $\bar{E}$ un fibré vectoriel adélique sur $\operatorname{Spec} k$. On a $\widehat{\operatorname{deg}} \bar{E}=\widehat{\operatorname{deg}} J(\bar{E})+n \log \operatorname{vr}(\bar{E}) \quad$ et $\quad \widehat{\operatorname{deg}} L(\bar{E})=\widehat{\operatorname{deg}} \bar{E}+n \log \widetilde{\operatorname{vr}}(\bar{E})$.

DÉmonstration. On identifie $E$ à $k^{n}$ en choisissant une $k$-base quelconque de $E$. La contribution au degré adélique de $\bar{E}$ des places ultramétriques est exactement la même que celle de $J(\bar{E})$, par définition de ce dernier fibré. En une place archimédienne $v$ de $k$, le quotient volumique de $\left(k_{v}^{n},\|\cdot\|_{v}\right)$ intervient à travers l'égalité :

$$
\begin{aligned}
& \frac{\operatorname{vol}\left(\left\{x \in k_{v}^{n} ;\|x\|_{v} \leq 1\right\}\right)}{\operatorname{vol}\left(\left\{x \in k_{v}^{n} ;|x|_{2} \leq 1\right\}\right)} \\
& \quad=\frac{\operatorname{vol}\left(\left\{x \in k_{v}^{n} ;|x|_{J(\bar{E}), v} \leq 1\right\}\right)}{\operatorname{vol}\left(\left\{x \in k_{v}^{n} ;|x|_{2} \leq 1\right\}\right)} \cdot \operatorname{vr}\left(k_{v}^{n},\|\cdot\|_{v}\right)^{\operatorname{dim}_{\mathrm{R}} k_{v}^{n}} .
\end{aligned}
$$

La proposition s'en déduit alors car $\operatorname{dim}_{\mathbf{R}} k_{v}^{n}=n_{v} n$. La même démarche avec l'ellipsoïde de Löwner permet de montrer l'autre égalité.

Par ailleurs, pour tout $\varepsilon>0$ et grâce à l'encadrement (6), on a l'existence d'une norme hermitienne |.| $\left.\right|_{\varepsilon, v}$ sur $E \otimes_{k} k_{v}$ ( $v$ archimédienne) telle que, pour tout sous-espace vectoriel $E^{\prime}$ de $E$,

$$
\left(E^{\prime},\left(|\cdot|_{\varepsilon, v}\right)_{v}\right) \preceq \overline{E^{\prime}} \preceq\left(E^{\prime},\left(\mathrm{d}\left(E \otimes_{k} k_{v}, \ell_{n, k_{v}}^{2}\right)(1+\varepsilon)|\cdot|_{\varepsilon, v}\right)_{v}\right)
$$

(les normes aux places ultramétriques des fibrés adéliques de gauche et de droite sont celles de $\bar{E}$ ).

Notation. On désigne par $\bar{E}_{\varepsilon}$ le fibré vectoriel adélique d'espace vectoriel sous-jacent $E$ et dont les normes sont

$$
\|\cdot\|_{\bar{E}_{\varepsilon}, v}:= \begin{cases}\|\cdot\|_{E, v} & \text { si } v \text { est ultramétrique, } \\ |\cdot|_{\varepsilon, v} & \text { si } v \text { est archimédienne. }\end{cases}
$$

On notera aussi plus simplement $|\cdot|_{\varepsilon}$ la collection $\left(\|\cdot\|_{\bar{E}_{\varepsilon}, v}\right)_{v}$ des normes 
de $\bar{E}_{\varepsilon}$. Il est bien clair que cette définition repose sur le choix des métriques hermitiennes $|\cdot|_{\varepsilon, v}$ et que le fibré $\bar{E}_{\varepsilon}$ n'est pas déterminé uniquement par $\bar{E}$ et $\varepsilon$.

Les définitions même des fibrés de John et Löwner entraînent $\widehat{\operatorname{deg}}_{\mathrm{n}} \bar{E}_{\varepsilon} \leq \widehat{\operatorname{deg}}_{\mathrm{n}} J(\bar{E})$ et

$$
\widehat{\operatorname{deg}}_{\mathrm{n}} L(\bar{E}) \leq \widehat{\operatorname{deg}}_{\mathrm{n}}\left(E,\left(\mathrm{~d}\left(E \otimes_{k} k_{v}, \ell_{n, k_{v}}^{2}\right)(1+\varepsilon)|\cdot|_{\varepsilon, v}\right)_{v}\right),
$$

puis, en faisant tendre $\varepsilon$ vers 0 , l'on obtient

$$
0 \leq \widehat{\operatorname{deg}}_{\mathrm{n}} L(\bar{E})-\widehat{\operatorname{deg}}_{\mathrm{n}} J(\bar{E}) \leq \frac{n}{D} \log \Delta(\bar{E}) .
$$

De l'encadrement (28), on déduit aussi

$$
-\frac{n}{D} \log \Delta(\bar{E})-n \log (1+\varepsilon) \leq \widehat{\operatorname{deg}}_{\mathrm{n}} \overline{E^{\prime}}-\widehat{\operatorname{deg}}_{\mathrm{n}}\left(E^{\prime},\left(|\cdot|_{\varepsilon, v}\right)_{v}\right) \leq 0 .
$$

Ceci permet d'approcher le degré adélique de $\overline{E^{\prime}}$ par le degré d'un fibré adélique hermitien, avec un terme d'erreur de l'ordre de $-\frac{n}{D} \log \Delta(\bar{E})$. Cette observation sera constamment utilisée dans la suite.

Corollaire 4.10. Pour tout fibré vectoriel adélique $\bar{E}$ et toute norme tensorielle adélique hermitienne $\alpha$ d'ordre $\operatorname{dim} E$, on a

$$
\left|\widehat{\operatorname{deg}}_{\mathrm{n}} \bar{E}-\widehat{\operatorname{deg}}_{\mathrm{n}} \operatorname{det}_{\alpha} \bar{E}\right| \leq \widehat{\operatorname{deg}}_{\mathrm{n}} L(\bar{E})-\widehat{\operatorname{deg}}_{\mathrm{n}} J(\bar{E}) .
$$

DÉmonstration. La différence ne se situe éventuellement qu'en une place archimédienne $v$ de $k$. On a vu que dans ce cas $|\cdot|_{L(\bar{E}), v} \leq\|\cdot\|_{E, v} \leq|\cdot|_{J(\bar{E}), v}$. L'hypothèse $\alpha$ hermitienne et les lemmes 4.2 et 4.3 entraînent alors l'inégalité

$$
\widehat{\operatorname{deg}}_{\mathrm{n}} \operatorname{det} J(\bar{E}) \leq \widehat{\operatorname{deg}}_{\mathrm{n}} \operatorname{det}_{\alpha} \bar{E} \leq \widehat{\operatorname{deg}}_{\mathrm{n}} \operatorname{det} L(\bar{E}) .
$$

Le lemme 4.5 et la proposition 4.9 donnent

$$
-\frac{n}{D} \log \widetilde{\operatorname{vr}}(\bar{E}) \leq \widehat{\operatorname{deg}}_{\mathrm{n}} \bar{E}-\widehat{\operatorname{deg}}_{\mathrm{n}} \operatorname{det}_{\alpha} \bar{E} \leq \frac{n}{D} \log \operatorname{vr}(\bar{E}),
$$

qui est un encadrement un peu plus précis que celui annoncé puisque $\max \{\operatorname{vr}(\bar{E}), \widetilde{\operatorname{vr}}(\bar{E})\} \leq \operatorname{vr}(\bar{E}) \widetilde{\operatorname{vr}}(\bar{E})$.

On rappelle que $\bar{k}$ désigne une clôture algébrique de $k$.

DÉfinition 4.11. Soit $\bar{E}=\left(E,\left(\|\cdot\|_{v}\right)_{v}\right)$ un fibré vectoriel adélique. La hauteur d'un élément $x \in\left(E \otimes_{k} \bar{k}\right) \backslash\{0\}$ relative à $\bar{E}$, notée $h_{\bar{E}}(x)$, est la 
somme normalisée

$$
h_{\bar{E}}(x):=\frac{1}{D} \sum_{v} n_{v} \log \|x\|_{E, v}
$$

(la somme porte sur toutes les places $v$ de $k$ mais seuls un nombre fini de termes ne sont pas nuls).

C'est aussi l'opposé du degré normalisé du fibré en droites $\left(K . x,\left(\|\cdot\|_{E, v}\right)_{v}\right)$ où $K$ est une extension finie de $k$ sur laquelle est défini $x$. La hauteur de $x$ ne dépend pas du choix de $K$ en raison de la formule $\sum_{w \mid v}\left[K_{w}: k_{v}\right]=[K: k]$ (voir aussi le paragraphe suivant). La terminologie est cohérente avec la notion de hauteur au sens usuel grâce au résultat suivant.

Proposition 4.12. Soit $a \in \mathbf{R}$. L'ensemble des droites $\{k . x ; x \in E$ et $\left.h_{\bar{E}}(x) \leq a\right\}$ est fini.

DÉmonstration. Soit $\left(e_{1}, \ldots, e_{n}\right)$ une $k$-base de $E$. Il existe une constante $\alpha>0$ (qui dépend de $\bar{E}$ et de $k$ ) telle que, pour toute place $v$ de $k$ et pour tout $x=\sum_{i=1}^{n} x_{i} e_{i} \in E$, on ait $\|x\|_{\bar{E}, v} \geq \alpha \max _{1 \leq i \leq n}\left\{\left|x_{i}\right|_{v}\right\}$. Aux places ultramétriques (et il suffit de considérer un nombre fini d'entre elles car $\bar{E}$ est adélique), cela résulte de la formule (15). Et aux places archimédiennes, il s'agit d'une conséquence de l'équivalence des normes en dimension finie. Si $x \neq 0$, la borne $h_{\bar{E}}(x) \leq a$ entraîne une majoration de la hauteur de Weil $\mathrm{du}$ point projectif $\left(x_{1}: \cdots: x_{n}\right)$ et donc un nombre fini de tels points (théorème de Northcott) et de vecteurs $x$ correspondant.

Proposition 4.13. Soit $\bar{E}$ un fibré vectoriel adélique sur Spec $k$ de dimension $n$ et $\alpha$ une norme tensorielle adélique d'ordre $n$. Soit $\left(e_{1}, \ldots, e_{n}\right)$ une k-base de E. Alors on a

$$
0 \leq \widehat{\operatorname{deg}}_{\mathrm{n}} \operatorname{det}_{\alpha} \bar{E}+h_{\bar{E}}\left(e_{1}\right)+\cdots+h_{\bar{E}}\left(e_{n}\right) .
$$

De plus, on a

$$
-\frac{n}{D} \log \Delta(\bar{E}) \leq \widehat{\operatorname{deg}}_{\mathrm{n}} \bar{E}+h_{\bar{E}}\left(e_{1}\right)+\cdots+h_{\bar{E}}\left(e_{n}\right) .
$$

DÉmONSTRATION. La première assertion est une conséquence de l'inégalité d'Hadamard

$$
\left\|e_{1} \wedge \cdots \wedge e_{n}\right\|_{\operatorname{det}_{\alpha} \bar{E}, v} \leq\left\|e_{1} \otimes \cdots \otimes e_{n}\right\|_{\bar{E}^{\otimes \alpha n}, v} \leq \prod_{i=1}^{n}\left\|e_{i}\right\|_{E, v},
$$


vraie pour toute place $v$ de $k$. En effet, la première majoration vient de la définition de norme quotient et la seconde est la propriété (i) de la définition 2.10 des normes tensorielles (il y a même égalité). Pour obtenir la seconde assertion, on choisit pour $\alpha$ une norme hermitienne (construite, par exemple, au moyen de la norme hermitienne $g_{2}$ de Chevet-Saphard) dans le corollaire 4.10 et on utilise l'estimation (29).

Il est souvent utile de connaître également une majoration de la somme des hauteurs d'une base de $E \otimes \bar{k}$ où $\bar{k}$ désigne une clôture algébrique de $k$. C'est l'objet du lemme de Siegel absolu suivant, établi dans le cas des corps de fonctions par D. Roy \& J.L. Thunder [27] et issu d'un énoncé dû à $\mathrm{S}$. Zhang [41] dans le cas d'un corps de nombres (les deux étant écrits dans le cas hermitien).

THÉORÈme 4.14. Soit $\bar{E}$ un fibré vectoriel adélique, de dimension $n \geq 1$. Posons $\delta:=0$ si $k$ est un corps de fonctions et $\delta:=1$ si $k$ est un corps de nombres. Pour tout $\varepsilon>0$, il existe une base $\left(e_{1}, \ldots, e_{n}\right)$ de $E \otimes \bar{k}$ telle que

$$
h_{\bar{E}}\left(e_{1}\right)+\cdots+h_{\bar{E}}\left(e_{n}\right)+\widehat{\operatorname{deg}}_{\mathrm{n}} \bar{E} \leq \frac{\delta n}{2} \log n+\frac{n}{D} \log \operatorname{vr}(\bar{E})+\varepsilon .
$$

Dans le cas d'un corps de nombres, la preuve complète se trouve dans [17]. Il est possible de demander que la base $\left(e_{1}, \ldots, e_{n}\right)$ soit définie sur $k$ et, en contrepartie, de rajouter un terme dépendant du discriminant absolu $D_{k}$ de $k$ dans le membre de droite de l'inégalité (31) (voir [27]). Ce terme est $(n / D) \log \left|D_{k}\right|$ si $k$ est un corps de nombres. Le cas général d'un fibré vectoriel adélique non nécessairement hermitien s'obtient en appliquant le théorème ci-dessus au fibré (hermitien) de John $J(\bar{E})$ et en utilisant $\bar{E} \preceq J(\bar{E})$ ainsi que la proposition 4.9.

\section{3. - Extension des scalaires.}

En toute généralité, le degré adélique normalisé n'est pas un invariant stable par extension des scalaires. Si l'on considère par exemple le fibré vectoriel adélique $\left(\mathbf{Q}^{n},|\cdot|_{\infty}\right)$, la formule (4) entraîne l'égalité

$$
\widehat{\operatorname{deg}}_{\mathrm{n}}\left(\mathbf{Q}^{n},|\cdot|_{\infty}\right)=\log \frac{\Gamma\left(1+\frac{n}{2}\right)}{\pi^{n / 2}} .
$$

Or si l'on choisit l'extension quadratique $\mathbf{Q}(i)$, qui possède une seule place 
complexe et aucune réelle, un rapide calcul donne

$$
\widehat{\operatorname{deg}}_{\mathrm{n}}\left(\mathbf{Q}(i)^{n},|\cdot|_{\infty}\right)=\frac{1}{2} \log n !,
$$

nombre toujours différent de celui de la formule précédente, dès que $n \geq 2$. Cette anomalie se voit également au moyen de la formule asymptotique (24) dont les coefficients $b$ et $c$ dépendent en général de $r_{1}$ et $r_{2}$ et non seulement de la somme $r_{1}+2 r_{2}=[k: \mathbf{Q}]$.

L'explication de ce phénomène est l'observation suivante, qui, réduite à l'essentiel, marque la différence entre le volume d'un cube et celui d'une boule.

LEMME 4.15. Soit $\|$.$\| une norme sur \mathbf{C}^{n}$, invariante par conjugaison complexe. Il existe une constante $\kappa=\kappa(n,\|\|$.$) , comprise entre 4^{-n}$ et $4^{n}$, telle que

$$
\frac{\operatorname{vol}\left(\left\{x \in \mathbf{C}^{n} ;\|x\| \leq 1\right\}\right)}{\operatorname{vol}\left(\left\{x \in \mathbf{C}^{n} ;|x|_{2} \leq 1\right\}\right)}=\kappa\left(\frac{\operatorname{vol}\left(\left\{x \in \mathbf{R}^{n} ;\|x\| \leq 1\right\}\right)}{\operatorname{vol}\left(\left\{x \in \mathbf{R}^{n} ;|x|_{2} \leq 1\right\}\right)}\right)^{2} .
$$

La notation vol désigne indifféremment une mesure de Haar (quelconque) sur $\mathbf{R}^{n}$ ou $\mathbf{C}^{n}$. Si la norme $\|$.$\| est hermitienne alors \kappa=1$.

DÉmonstration. Il s'agit d'une simple conséquence des inégalités :

$$
\max \left\{\left\|x_{1}\right\|,\left\|x_{2}\right\|\right\} \leq \frac{1}{2}\left(\left\|x_{1}+i x_{2}\right\|+\left\|x_{1}-i x_{2}\right\|\right)=\left\|x_{1}+i x_{2}\right\|
$$

et $\left\|x_{1}+i x_{2}\right\| \leq\left\|x_{1}\right\|+\left\|x_{2}\right\| \leq 2 \max \left\{\left\|x_{1}\right\|,\left\|x_{2}\right\|\right\}$, pour tous $x_{1}, x_{2} \in \mathbf{R}^{n}$. Le résultat s'ensuit en choisissant sur $\mathbf{C}^{n}$ la mesure de Haar produit $\operatorname{vol}_{\mathbf{R}^{n}} \otimes \operatorname{vol}_{\mathbf{R}^{n}}$, obtenue au moyen de l'isomorphisme naturel $\mathbf{C}^{n} \simeq \mathbf{R}^{n} \times \mathbf{R}^{n}$. Si la norme est hermitienne, elle est déterminée par la donnée d'une matrice symétrique $A \in M_{n}(\mathbf{R})$ (il n'y a pas de partie imaginaire car la norme induite doit être invariante par conjugaison complexe). Les deux membres de l'équation (32) valent alors $(\operatorname{det} A)^{-1}$ et $\kappa=1$.

Remarque 4.16. Les calculs exacts des volumes de $b_{n, \mathbf{C}}^{2}$ et $b_{n, \mathbf{R}}^{2}$ données par les formules (4) et (5) fournissent l'encadrement plus précis

$$
\frac{1}{4^{n}} \cdot \frac{n !}{\Gamma\left(1+\frac{n}{2}\right)^{2}} \leq \kappa \leq \frac{n !}{\Gamma\left(1+\frac{n}{2}\right)^{2}} .
$$


On peut noter que la formule de Stirling (voir note au bas de la page 29) donne l'équivalent asymptotique

$$
\frac{n !}{\Gamma\left(1+\frac{n}{2}\right)^{2}} \sim \frac{2^{n+1 / 2}}{\sqrt{\pi n}} \quad \text { lorsque } n \longrightarrow+\infty .
$$

Après ces considérations, nous pouvons énoncer le résultat principal de ce paragraphe.

Proposition 4.17. Soit $K$ une extension finie de $k$ et $\bar{E}$ un fibré vectoriel adélique sur $k$, de dimension $n$. Alors

$$
\left|\widehat{\operatorname{deg}}_{\mathrm{n}} \bar{E}_{K}-\widehat{\operatorname{deg}}_{\mathrm{n}} \bar{E}\right| \leq n \log (4) .
$$

Si, de plus, $\bar{E}$ est un fibré adélique hermitien alors $\widehat{\operatorname{deg}}_{\mathrm{n}} \bar{E}_{K}=\widehat{\operatorname{deg}}_{\mathrm{n}} \bar{E}$.

DÉmonstration. Quitte à fixer une $k$-base de $E$, on peut supposer $E=k^{n}$. On distingue deux cas.

a) Soit v une place ultramétrique de $k$. On a vu qu'il existe une matrice $c_{v} \in \mathrm{GL}_{n}\left(k_{v}\right)$ telle que:

$$
\forall x=\left(x_{1}, \ldots, x_{n}\right) \in \mathbf{C}_{v}^{n}, \quad\|x\|_{E, v}=\max _{1 \leq i \leq n}\left\{\left|\left(c_{v} \cdot x\right)_{i}\right|_{v}\right\} .
$$

Notons $B_{v}:=\left\{x \in k_{v}^{n} ;\|x\|_{v} \leq 1\right\}$. Cet ensemble est l'image par $c_{v}^{-1}$ de $\mathcal{O}_{v}^{n}$ et, d'après les rappels du paragraphe 2.1 , on a

$$
\operatorname{vol}_{v}\left(B_{v}\right)=\left|\operatorname{det} c_{v}\right|_{v}^{-n_{v}} \operatorname{vol}_{v}\left(\mathcal{O}_{v}^{n}\right)
$$

(ici vol $v_{v}$ est une mesure de Haar sur $k_{v}^{n}$ ). Soit $w$ une place de $K$ au-dessus de $v$. Le complété $K_{w}$ est un $k_{v}$-espace vectoriel de dimension $\left[K_{w}: k_{v}\right.$, et si l'on note $B_{w}$ la boule unité fermée dans $K_{w}^{n}$, on a

$$
\frac{\operatorname{vol}_{w}\left(B_{w}\right)}{\operatorname{vol}_{w}\left(\mathcal{O}_{w}^{n}\right)}=\left|\operatorname{det} c_{v}\right|_{v}^{-n_{w}}=\left(\frac{\operatorname{vol}_{v}\left(B_{v}\right)}{\operatorname{vol}_{v}\left(\mathcal{O}_{v}^{n}\right)}\right)^{\left[K_{w}: k_{v}\right]} .
$$

Comme cela est rappelé dans les préliminaires, l'on dispose de l'isomorphisme d'espaces vectoriels topologiques

$$
K \otimes_{k} k_{v} \simeq \prod_{w \mid v} K_{w},
$$


qui, outre l'égalité des dimensions $[K: k]=\sum_{w \mid v}\left[K_{w}: k_{v}\right]$, entraîne

$$
\begin{aligned}
& \frac{\operatorname{vol}\left(\left\{x \in\left(K \otimes k_{v}\right)^{n} ;\|x\|_{v} \leq 1\right\}\right)}{\left.\operatorname{vol}\left(\left\{x \in\left(K \otimes k_{v}\right)^{n} ; \max _{1 \leq i \leq n}\left\{\left|x_{i}\right|_{v}\right\} \leq 1\right\}\right\}\right)} \\
& =\prod_{w \mid v} \frac{\operatorname{vol}_{w}\left(B_{w}\right)}{\operatorname{vol}_{w}\left(\mathcal{O}_{w}^{n}\right)}=\left(\frac{\operatorname{vol}_{v}\left(B_{v}\right)}{\operatorname{vol}_{v}\left(\mathcal{O}_{v}^{n}\right)}\right)^{[K: k]}
\end{aligned}
$$

(la mesure vol sur $\left(K \otimes k_{v}\right)^{n}$ peut être choisie comme l'image réciproque de la mesure produit $\otimes_{w \mid v} \operatorname{vol}_{w}$ sur $\prod_{w \mid v} K_{w}^{n}$ par l'isomorphisme (34)). Soulignons que cette égalité ne dépend ni du choix de l'isomorphisme (34), ni du choix des mesures $\operatorname{vol}_{w}, \operatorname{vol}_{v}$ ou bien encore vol. Ainsi le comportement du quotient des volumes aux places ultramétriques par extension des scalaires est exactement celui souhaité et les parties (aux places) finies des degrés adéliques normalisés de $\bar{E}_{K}$ et $\bar{E}$ sont identiques. En particulier, ces degrés (dans leur totalité) sont égaux si $k$ est un corps de fonctions.

b) Soit $v$ une place archimédienne de $k$. Comme précédemment, soit $w$ une place de $K$ au-dessus de $v$. Si $w$ et $v$ sont de même nature (toutes les deux réelles ou complexes), le quotient des volumes

$$
\frac{\operatorname{vol}\left(\left\{x \in k_{v}^{n} ;\|x\|_{v} \leq 1\right\}\right)}{\operatorname{vol}\left(\left\{x \in k_{v}^{n} ;|x|_{2} \leq 1\right\}\right)}
$$

reste inchangé. En revanche, si $v$ est réelle et $w$ est complexe, nous sommes dans le cas du figure du lemme 4.15 et la proposition 4.17 s'en déduit immédiatement (le cas d'égalité lorsque $\bar{E}$ est un fibré vectoriel hermitien provenant du cas d'égalité $\kappa=1$ dans ce même lemme).

REMARque 4.18. L'argument qui est à la fin de cette démonstration permet d'établir en réalité une borne plus faible pour la différence des degrés puisqu'il suffit de considérer les couples de places $(v, w)$ telles que $w \mid v$ et qui ne sont pas de même nature. Si $N(k, K)$ est le nombre de tels couples, la majoration (33) reste vraie en multipliant $n \log (4)$ par le quotient $N(k, K) /[K: \mathbf{Q}] \leq 1$.

\section{4. - Somme directe}

Comme nous l'avons vu au paragraphe 3.3, il est possible de définir la somme directe $\bar{E} \oplus_{\varsigma} \bar{F}$ de deux fibrés vectoriels adéliques, relative à une famille $\varsigma=\left(\varsigma_{v}\right)_{\left.v\right|_{\infty}}$ de normes (particulières) sur $\mathbf{R}^{2}$. La proposition suivante évalue le degré d'une telle somme directe. 
Proposition 4.19. Soit $\bar{E}$ et $\bar{F}$ des fibrés vectoriels adéliques sur Spec $k$, de dimensions respectives $n$ et $m$. On a alors

$$
\left|\widehat{\operatorname{deg}}_{\mathrm{n}}\left(\bar{E} \oplus_{\varsigma} \bar{F}\right)-\widehat{\operatorname{deg}}_{\mathrm{n}} \bar{E}-\widehat{\operatorname{deg}}_{\mathrm{n}} \bar{F}\right| \leq \log \left(\begin{array}{c}
n+m \\
n
\end{array}\right) .
$$

Pour la somme hermitienne (c.-̀̀-d. $\varsigma_{v}(x, y)=\left(x^{2}+y^{2}\right)^{1 / 2}$ aux places archimédiennes $v$ de $k$ ), on a l'égalité $\widehat{\operatorname{deg}}_{\mathrm{n}}\left(\bar{E} \oplus_{2} \bar{F}\right)=\widehat{\operatorname{deg}}_{\mathrm{n}} \bar{E}+\widehat{\operatorname{deg}}_{\mathrm{n}} \bar{F}$.

En particulier, si $k$ est un corps de fonctions, on a $\widehat{\operatorname{deg}}_{\mathrm{n}}(\bar{E} \oplus \bar{F})=$ $=\widehat{\operatorname{deg}}_{\mathrm{n}} \bar{E}+\widehat{\operatorname{deg}}_{\mathrm{n}} \bar{F}$.

DÉmonstration. En vertu de l'encadrement (8), on a $\bar{E} \oplus_{\infty} \bar{F} \preceq$ $\preceq \bar{E} \oplus_{\varsigma} \bar{F} \preceq \bar{E} \oplus_{1} \bar{F}$ et donc

$$
\widehat{\operatorname{deg}}\left(\bar{E} \oplus_{1} \bar{F}\right) \leq \widehat{\operatorname{deg}}\left(\bar{E} \oplus_{\varsigma} \bar{F}\right) \leq \widehat{\operatorname{deg}}\left(\bar{E} \oplus_{\infty} \bar{F}\right)
$$

(lemme 4.2). Or pour $p \in[1,+\infty]$ on dispose d'une formule exacte pour $\widehat{\operatorname{deg}}\left(\bar{E} \oplus_{p} \bar{F}\right)$. En effet, si l'on applique l'égalité (10), à $E_{v} \oplus_{p} F_{v}$ où $v$ est une place archimédienne de $k$, la différence $\widehat{\operatorname{deg}}\left(\bar{E} \oplus_{p} \bar{F}\right)-\widehat{\operatorname{deg}} \bar{E}-\widehat{\operatorname{deg}} \bar{F}$ égale

$$
\sum_{v \text { archimédienne }} \log \frac{\Gamma\left(1+\frac{n n_{v}}{p}\right) \Gamma\left(1+\frac{m n_{v}}{p}\right) \Gamma\left(1+\frac{(m+n) n_{v}}{2}\right)}{\Gamma\left(1+\frac{(m+n) n_{v}}{p}\right) \Gamma\left(1+\frac{n n_{v}}{2}\right) \Gamma\left(1+\frac{m n_{v}}{2}\right)} .
$$

Il n'y a pas de contributions aux places ultramétriques car, par choix de la norme sup, la boule unité de $E_{v} \oplus_{p} F_{v}$ est le produit des boules unités de $E_{v}$ et $F_{v}$. Et les volumes se multiplient également pourvu que l'on choisisse la mesure produit $\operatorname{vol}_{E, v} \otimes \operatorname{vol}_{F, v}$ sur la somme directe $E_{v} \oplus F_{v}$. On obtient ainsi au passage l'égalité $\widehat{\operatorname{deg}}_{\mathrm{n}}\left(\bar{E} \oplus_{2} \bar{F}\right)=\widehat{\operatorname{deg}}_{\mathrm{n}} \bar{E}+\widehat{\operatorname{deg}}_{\mathrm{n}} \bar{F}$ lorsque $p=2$. La majoration du degré de $\bar{E} \oplus_{\infty} \bar{F}$ s'obtient alors en remarquant

$$
\frac{\Gamma\left(1+\frac{m+n}{2}\right)}{\Gamma\left(1+\frac{n}{2}\right) \Gamma\left(1+\frac{m}{2}\right)} \leq\left(\begin{array}{c}
n+m \\
n
\end{array}\right)
$$

majoration que l'on utilise aux places réelles de $k$. Quant à la minoration du degré de $\bar{E} \oplus_{1} \bar{F}$, on se sert de $\Gamma\left(1+\frac{m+n}{2}\right) \geq \Gamma\left(1+\frac{n}{2}\right) \Gamma\left(1+\frac{m}{2}\right)$ (places réelles) et de $\left(\begin{array}{c}2 n+2 m \\ 2 n\end{array}\right) \leq\left(\begin{array}{c}n+m \\ n\end{array}\right)^{3}$ (places complexes). Cette dernière estimation peut se démontrer par récurrence sur $m$ et les précédentes au moyen de la formule d'Euler pour la fonction Gamma. 
Remarque 4.20. En observant que, pour tout $x \in \mathbf{C}^{n}$, on a $|x|_{2} \leq|x|_{p}$ si $p \leq 2$ et $|x|_{2} \geq|x|_{p}$ si $p \geq 2$, on obtient aisément les majorations

$$
\widehat{\operatorname{deg}}_{\mathrm{n}}\left(\bar{E} \oplus_{p} \bar{F}\right) \leq \widehat{\operatorname{deg}}_{\mathrm{n}} \bar{E}+\widehat{\operatorname{deg}}_{\mathrm{n}} \bar{F} \quad \text { si } p \leq 2
$$

et

$$
\widehat{\operatorname{deg}}_{\mathrm{n}} \bar{E}+\widehat{\operatorname{deg}}_{\mathrm{n}} \bar{F} \leq \widehat{\operatorname{deg}}_{\mathrm{n}}\left(\bar{E} \oplus_{p} \bar{F}\right) \quad \text { si } p \geq 2 .
$$

4.5. - Dualité.

Proposition 4.21. Il existe une constante absolue c $>0$ telle que, pour tout fibré vectoriel adélique $\bar{E}$ de dimension $n$, on a

$$
\widehat{\operatorname{deg}}_{\mathrm{n}} \bar{E}+\widehat{\operatorname{deg}}_{\mathrm{n}} \bar{E}^{\vee} \in[-c n, 0] .
$$

Si $\bar{E}$ est un fibré adélique hermitien alors $\widehat{\operatorname{deg}}_{\mathrm{n}} \bar{E}^{\mathrm{v}}=-\widehat{\operatorname{deg}}_{\mathrm{n}} \bar{E}$.

DÉmonstration. On peut supposer $E=k^{n}$. Soit $v$ une place de $k$. S'il existe une matrice $u_{v} \in \mathrm{GL}_{n}\left(k_{v}\right)$ telle que $\left(k_{v}^{n},\|\cdot\|_{v}\right)=u_{v}\left(k_{v}^{n},|\cdot|_{2, v}\right)$ alors le quotient des volumes

$$
\frac{\operatorname{vol}\left(\mathbf{B}\left(k_{v}^{n},\|\cdot\|_{v}\right)\right)}{\operatorname{vol}\left(\mathbf{B}\left(k_{v}^{n},|\cdot|_{2, v}\right)\right)}
$$

égale $\left|\operatorname{det} u_{v}\right|_{v}^{-n_{v}}$. Il suffit alors d'observer que les normes duales $\|\cdot\|_{v}^{v}$ et $|\cdot|_{2, v}^{v}$ sont reliées par la matrice inverse $u_{v}^{-1}$ et le quotient des volumes pour les espaces duaux est inversé. En particulier cela assure l'égalité $\widehat{\operatorname{deg}} \bar{E}+$ $+\widehat{\operatorname{deg}} \bar{E}^{\vee}=0$ si $\bar{E}$ est un fibré adélique hermitien. Dans le cas général, comme il ne reste que les places archimédiennes à traiter, on utilise le théorème 2.3 en chacune de ces places (avec $n=\operatorname{dim}_{\mathbf{R}} E_{v}$ ) et le résultat s'ensuit.

\section{6. - Quotient.}

Proposition 4.22. Soit $\bar{E}_{2} \subseteq \bar{E}_{1}$ deux sous-fibrés adéliques de $\bar{E}$. Alors on a

$$
\left|\widehat{\operatorname{deg}}_{\mathrm{n}} \bar{E}_{1}-\widehat{\operatorname{deg}}_{\mathrm{n}} \bar{E}_{2}-\widehat{\operatorname{deg}}_{\mathrm{n}} \overline{E_{1} / E_{2}}\right| \leq \frac{\operatorname{dim} E_{1}}{D} \log \Delta(\bar{E}) .
$$


DÉmonstration. Si $\bar{E}$ est un fibré adélique hermitien, la métrique quotient sur $E_{1} / E_{2}$ coïncide en une place archimédienne $v$ avec la métrique sur l'orthogonal de $E_{2, v}$ relatif au produit hermitien $(,)_{E, v}$ de $E_{v}$. En une place ultramétrique, la métrique quotient est celle d'un supplémentaire quelconque de $E_{2}$ dans $E_{1}$. Ainsi le fibré $\bar{E}_{1}$ est isomorphe isométriquement à $\bar{E}_{2} \oplus_{2} \overline{E_{1} / E_{2}}$ et la proposition 4.19 donne l'égalité

$$
\widehat{\operatorname{deg}}_{\mathrm{n}} \bar{E}_{1}=\widehat{\operatorname{deg}}_{\mathrm{n}} \bar{E}_{2}+\widehat{\operatorname{deg}}_{\mathrm{n}} \overline{E_{1} / E_{2}} .
$$

La propriété étant établie dans le cas hermitien, le passage au cas général s'effectue grâce aux fibrés de John et Löwner associés à $\bar{E}_{1}$. Comme $\|\cdot\|_{E_{1}, v} \leq|\cdot|_{J\left(\bar{E}_{1}\right), v}$ pour toute place $v$ de $k$, cela entraîne

$$
\widehat{\operatorname{deg}}_{\mathrm{n}}\left(E_{2},|\cdot|_{J\left(\bar{E}_{1}\right), E_{2}}\right) \leq \widehat{\operatorname{deg}}_{\mathrm{n}} \bar{E}_{2}
$$

et

$$
\widehat{\operatorname{deg}}_{\mathrm{n}}\left(E_{1} / E_{2},|\cdot|_{J\left(\bar{E}_{1}\right), E_{1} / E_{2}}\right) \leq \widehat{\operatorname{deg}}_{\mathrm{n}}\left(\overline{E_{1} / E_{2}}\right)
$$

où $|\cdot|_{J\left(\bar{E}_{1}\right), E_{2}}\left(\right.$ resp. $\left.|\cdot|_{J\left(\bar{E}_{1}\right), E_{1} / E_{2}}\right)$ désigne la famille des normes de John de $\bar{E}_{1}$ restreintes à $E_{2}$ (resp. normes quotient sur $E_{1} / E_{2}$ issues des normes de John de $\bar{E}_{1}$ ). L'on obtient ainsi

$$
\begin{aligned}
& \widehat{\operatorname{deg}}_{\mathrm{n}} \bar{E}_{1}-\widehat{\operatorname{deg}}_{\mathrm{n}} \bar{E}_{2}-\widehat{\operatorname{deg}}_{\mathrm{n}} \overline{E_{1} / E_{2}} \\
& \quad \leq \widehat{\operatorname{deg}}_{\mathrm{n}} \bar{E}_{1}-\left(\widehat{\operatorname{deg}}_{\mathrm{n}}\left(E_{2},|\cdot|_{J\left(\bar{E}_{1}\right), E_{2}}\right)+\widehat{\operatorname{deg}}_{\mathrm{n}}\left(E_{1} / E_{2},|\cdot|_{J\left(\bar{E}_{1}\right), E_{1} / E_{2}}\right)\right)
\end{aligned}
$$

et l'expression dans cette dernière parenthèse égale $\widehat{\operatorname{deg}}_{\mathrm{n}} J\left(\bar{E}_{1}\right)$ car $J\left(\bar{E}_{1}\right)$ est un fibré adélique hermitien. On raisonne de la même façon avec $L\left(\overline{E_{1}}\right)$ pour la majoration en sens inverse. Grâce à (29), on obtient la borne

$$
\begin{aligned}
\left|\widehat{\operatorname{deg}}_{\mathrm{n}} \bar{E}_{1}-\widehat{\operatorname{deg}}_{\mathrm{n}} \bar{E}_{2}-\widehat{\operatorname{deg}}_{\mathrm{n}} \overline{E_{1} / E_{2}}\right| & \leq \widehat{\operatorname{deg}}_{\mathrm{n}} L\left(\bar{E}_{1}\right)-\widehat{\operatorname{deg}}_{\mathrm{n}} J\left(\bar{E}_{1}\right) \\
& \leq \frac{\operatorname{dim} E_{1}}{D} \log \Delta\left(\bar{E}_{1}\right)
\end{aligned}
$$

et l'on conclut avec $\Delta\left(\bar{E}_{1}\right) \leq \Delta(\bar{E})$.

\section{7. - Somme.}

Soit $\bar{E}$ un fibré vectoriel adélique sur Spec $k$ et $E_{1}, E_{2}$ deux sous-espaces vectoriels de $E$, de dimensions respectives $n_{1}$ et $n_{2}$. Chacun des quatre espaces vectoriels $E_{1}, E_{2}, E_{1}+E_{2}, E_{1} \cap E_{2}$ est muni de la structure de sous-fibré vectoriel adélique induite par $\bar{E}$. 
Proposition 4.23. Avec les données ci-dessus, la différence des degrés adéliques normalisés

$$
\widehat{\operatorname{deg}}_{\mathrm{n}}\left(\overline{E_{1}+E_{2}}\right)+\widehat{\operatorname{deg}}_{\mathrm{n}} \overline{E_{1} \cap E_{2}}-\widehat{\operatorname{deg}}_{\mathrm{n}} \bar{E}_{1}-\widehat{\operatorname{deg}}_{\mathrm{n}} \bar{E}_{2}
$$

est supérieure à

$$
-\frac{\left(n_{1}+n_{2}\right)}{D} \log \Delta\left(\overline{E_{1}+E_{2}}\right)
$$

DÉmonstration. L'application naturelle $\imath: E_{2} /\left(E_{1} \cap E_{2}\right) \rightarrow\left(E_{1}+E_{2}\right) / E_{1}$ vérifie, pour toute place $v$ de $k$ et tout $x \in k_{v}$,

$$
\|\imath(x)\|_{\left(E_{1}+E_{2}\right) / E_{1}, v} \leq\|x\|_{E_{2} /\left(E_{1} \cap E_{2}\right), v} .
$$

Par conséquent, la boule unité du premier quotient est incluse dans celle du second et l'on a

$$
\widehat{\operatorname{deg}}_{\mathrm{n}}\left(\overline{E_{2} /\left(E_{1} \cap E_{2}\right)}\right) \leq \widehat{\operatorname{deg}}_{\mathrm{n}}\left(\overline{\left(E_{1}+E_{2}\right) / E_{1}}\right) .
$$

Si $\overline{E_{1}+E_{2}}$ est hermitien, la proposition 4.22 permet de conclure puisque dans ce cas le degré d'un quotient est la différence des degrés. Dans le cas général, on considère $\varepsilon>0$ et une norme hermitienne $|\cdot|_{\varepsilon, v}$ sur $\left(E_{1}+E_{2}\right) \otimes_{k} k_{v}$, ce qui permet de définir le fibré adélique hermitien $\overline{\left(E_{1}+E_{2}\right)_{\varepsilon}}(\operatorname{voir}(28))$. On a alors

$$
\begin{aligned}
\widehat{\operatorname{deg}}_{\mathrm{n}} \bar{E}_{1}+\widehat{\operatorname{deg}}_{\mathrm{n}} \bar{E}_{2} & \leq \widehat{\operatorname{deg}}_{\mathrm{n}}\left(E_{1},|\cdot|_{\varepsilon}\right)+\widehat{\operatorname{deg}}_{\mathrm{n}}\left(E_{2},|\cdot|_{\varepsilon}\right) \\
& \leq \widehat{\operatorname{deg}}_{\mathrm{n}}\left(E_{1}+E_{2},|\cdot|_{\varepsilon}\right)+\widehat{\operatorname{deg}}_{\mathrm{n}}\left(E_{1} \cap E_{2},|\cdot|_{\varepsilon}\right) .
\end{aligned}
$$

La première inégalité qui vient après (29) montre que le degré adélique normalisé de $\overline{\left(E_{1}+E_{2}\right)_{\varepsilon}}$ est plus petit que

$$
\widehat{\operatorname{deg}}_{\mathrm{n}} \overline{E_{1}+E_{2}}+\operatorname{dim}\left(E_{1}+E_{2}\right)\left(\log (1+\varepsilon)+\frac{1}{D} \log \Delta\left(\overline{E_{1}+E_{2}}\right)\right) .
$$

Il en est de même pour le degré de $\left(E_{1} \cap E_{2},|\cdot|_{\varepsilon}\right)$ en remplaçant $\operatorname{dim}\left(E_{1}+E_{2}\right)$ par $\operatorname{dim} E_{1} \cap E_{2}$. La proposition 4.23 s'ensuit en faisant tendre $\varepsilon$ vers 0 .

En notant $H(\bar{E})=\exp \left\{-\widehat{\operatorname{deg}}_{\mathrm{n}} \bar{E}\right\}$, cet énoncé peut se voir comme une généralisation de l'inégalité $H\left(\overline{E_{1}+E_{2}}\right) H\left(\overline{E_{1} \cap E_{2}}\right) \leq H\left(\overline{E_{1}}\right) H\left(\overline{E_{2}}\right)$ de W. Schmidt [32], T. Struppeck \& J.D. Vaaler [35], lorsque les métriques sont hermitiennes. 


\section{Théorie des pentes et pentes maximales.}

DÉFINITION 5.1. La pente adélique de $\bar{E}$ est

$$
\widehat{\mu}(\bar{E}):=\frac{\widehat{\operatorname{deg}} \bar{E}}{\operatorname{dim} E} .
$$

Par convention, si $E=\{0\}$, on pose $\widehat{\mu}(\bar{E}):=-\infty$. De plus, lorsque l'on divise ce nombre réel par le degré $D$ du corps $k$, on parle de pente adélique normalisée que l'on note $\widehat{\mu}_{\mathrm{n}}(\bar{E})$.

Cette quantité est un avatar adélique et logarithmique du rayon volumique

$$
\left(\frac{\operatorname{vol}(C)}{\operatorname{vol}\left(b_{n}^{2}\right)}\right)^{1 / n}
$$

d'un corps convexe $C$ de $\mathbf{R}^{n}$, utilisé dans l'étude des espaces de Banach.

Voici quelques propriétés que l'on déduit immédiatement de celles du degré adélique, démontrées dans le paragraphe précédent. Dans la liste qui suit, $\bar{E}$ désigne un fibré vectoriel adélique de dimension $n, \bar{E}_{\varepsilon}=\left(E,|\cdot|_{\varepsilon}\right)$ un fibré adélique hermitien associé à $\bar{E}$ (voir (28)) et $K$ est une extension finie de $k$.

\section{PROPRIÉtÉs:}

1) Pour tout sous-espace vectoriel $E^{\prime}$ de $E$, on a

$$
-\log (1+\varepsilon)-\frac{1}{D} \log \Delta(\bar{E}) \leq \widehat{\mu}_{\mathrm{n}}\left(\overline{E^{\prime}}\right)-\widehat{\mu}_{\mathrm{n}}\left(E^{\prime},|\cdot|_{\varepsilon}\right) \leq 0 .
$$

2) Il existe une constante absolue $c>0$ telle que $-c \leq \widehat{\mu}_{\mathrm{n}}(\bar{E})+$ $\widehat{\mu}_{\mathrm{n}}\left(\bar{E}^{\mathrm{V}}\right) \leq 0$.

3) $\left|\widehat{\mu}_{\mathrm{n}}\left(\bar{E}_{K}\right)-\widehat{\mu}_{\mathrm{n}}(\bar{E})\right| \leq 2 \log (2)$.

Si de plus $\bar{E}$ est un fibré adélique hermitien alors $\widehat{\mu}_{\mathrm{n}}\left(\bar{E}^{\mathrm{v}}\right)=-\widehat{\mu}_{\mathrm{n}}(\bar{E})$ et $\widehat{\mu}_{\mathrm{n}}\left(\bar{E}_{K}\right)=\widehat{\mu}_{\mathrm{n}}(\bar{E})$.

À ces propriétés s'ajoute une estimation de la pente d'un produit tensoriel.

Proposition 5.2. Soit $\ell \in \mathbf{N} \backslash\{0\}$. Pour tout $i \in\{1, \ldots, \ell\}$, soit $\bar{E}_{i}$ un fibré vectoriel adélique sur Spec $k$. Soit $\alpha$ une norme tensorielle adélique 
d'ordre $\ell$. Si tous les $\bar{E}_{i}$ sauf au plus un sont de dimension 1 alors $\widehat{\mu}\left(\otimes_{\alpha, i=1}^{\ell} \bar{E}_{i}\right)=\sum_{i=1}^{\ell} \widehat{\mu}\left(\bar{E}_{i}\right)$. En dimension quelconque, si a est hermitienne alors

$$
\left|\widehat{\mu}\left(\otimes_{\alpha, i=1}^{\ell} \bar{E}_{i}\right)-\sum_{i=1}^{\ell} \widehat{\mu}\left(\bar{E}_{i}\right)\right| \leq \sum_{i=1}^{\ell} \log \Delta\left(\bar{E}_{l}\right) .
$$

On déduit aussi de cette inégalité la relation $\widehat{\mu}\left(\otimes_{\alpha, i=1}^{\ell} \bar{E}_{i}\right)=\sum_{i=1}^{\ell} \widehat{\mu}\left(\bar{E}_{i}\right)$ lorsque tous les $\bar{E}_{i}$ et $\alpha$ sont hermitiens. Toutefois, la preuve que nous proposons commence par établir ce cas.

DÉmonstration. Le premier cas d'égalité lorsque par exemple $\operatorname{dim} E_{i}=1$ pour $2 \leq i \leq \ell$ s'obtient en observant que l'application $x \mapsto x \otimes e_{2} \otimes \cdots \otimes e_{\ell}$ de $\left(E,\left(\|\cdot\|_{E_{1}, v} \times \prod_{i=2}^{\ell}\left\|e_{i}\right\|_{E_{i}, v}\right)\right)$ dans $\otimes_{\alpha, i=1}^{\ell} \bar{E}_{i}$ est un isomorphisme isométrique ( $e_{i}$ est une $k$-base quelconque de $E_{i}$ ). Plaçons nous alors en dimension quelconque, avec $\alpha$ hermitienne. Supposons $\ell=2$ dans un premier temps. Si, en une place $v$ de $k$, les normes $\|\cdot\|_{E_{1}, v}$ et $\|\cdot\|_{E_{2}, v}$ sont simultanément hermitiennes ou ultramétriques, l'isomorphisme canonique

$$
\operatorname{det}\left(E_{1, v} \otimes E_{2, v}\right) \simeq \operatorname{det}\left(E_{1, v}\right)^{\otimes m} \otimes \operatorname{det}\left(E_{2, v}\right)^{\otimes n}
$$

est une isométrie. Par conséquent, lorsque $\bar{E}_{1}$ et $\bar{E}_{2}$ sont des fibrés adéliques hermitiens, on a

$$
\widehat{\operatorname{deg}} \operatorname{det}\left(\bar{E}_{1} \otimes \bar{E}_{2}\right)=m \widehat{\operatorname{deg}} \operatorname{det}\left(\bar{E}_{1}\right)+n \widehat{\operatorname{deg}} \operatorname{det}\left(\bar{E}_{2}\right)
$$

et le lemme 4.5 entraîne l'égalité $\widehat{\mu}\left(\bar{E}_{1} \otimes \bar{E}_{2}\right)=\widehat{\mu}\left(\bar{E}_{1}\right)+\widehat{\mu}\left(\bar{E}_{2}\right)$. Le résultat pour le produit de $\ell$ fibrés hermitiens s'en déduit par récurrence sur $\ell$. Une fois celui-ci établi, le passage au cas général s'effectue au moyen des fibrés de John et Löwner associés aux $\bar{E}_{i}$. Grâce au lemme 4.3 , on a

$$
\otimes_{i=1}^{\ell} L\left(\bar{E}_{i}\right) \preceq \otimes_{\alpha, i=1}^{\ell} \bar{E}_{i} \preceq \otimes_{i=1}^{\ell} J\left(\bar{E}_{i}\right) .
$$

On en déduit un encadrement de $\widehat{\mu}\left(\otimes_{\alpha, i=1}^{\ell} \bar{E}_{i}\right)$ via le lemme 4.2. L'hypothèse $\alpha$ hermitienne permet d'utiliser le cas hermitien établi juste avant et les formules de la proposition 4.9 conduisent à l'estimation

$$
\left|\widehat{\mu}\left(\otimes_{\alpha, i=1}^{\ell} \bar{E}_{i}\right)-\sum_{i=1}^{\ell} \widehat{\mu}\left(\bar{E}_{i}\right)\right| \leq \sum_{i=1}^{\ell}\left\{\widehat{\mu}\left(L\left(\bar{E}_{i}\right)\right)-\widehat{\mu}\left(J\left(\bar{E}_{i}\right)\right)\right\} .
$$

On conclut avec (29). 
5.1. - Existence de la pente maximale.

Proposition 5.3. Soit $\bar{E}$ un fibré vectoriel adélique sur Spec $k$. Il existe une constante $c(\bar{E}, k)$ telle que, pour tout sous-fibré vectoriel adélique $\bar{F}$ de $\bar{E}$, on a $\widehat{\mu}_{\mathrm{n}}(\bar{F}) \leq c(\bar{E}, k)$. Plus précisément, pour tout nombre réel a, il n'existe qu'un nombre fini de sous-fibrés vectoriels adéliques $\bar{F}$ de $\bar{E}$ tels que $\widehat{\mu}_{\mathrm{n}}(\bar{F}) \geq a$.

DÉmonstration. Soit $\bar{F} \subseteq \bar{E}$ et $m=\operatorname{dim} F$. Si $\bar{E}$ est un fibré adélique hermitien alors il en est de même pour $\bar{F}$ et l'on a

$$
\widehat{\mu}_{\mathrm{n}}(\bar{F})=\frac{\widehat{\operatorname{deg}}_{\mathrm{n}} \operatorname{det} \bar{F}}{\operatorname{dim} F}=-\frac{h_{\wedge^{m} \bar{E}}\left(f_{1} \wedge \cdots \wedge f_{m}\right)}{m}
$$

où $f_{1}, \ldots, f_{m}$ est une $k$-base de $\bar{F}$ et $h_{\wedge^{m} \bar{E}}$ est la hauteur sur les éléments de $\bigwedge^{m} \bar{E}$ donnée par la définition 4.11. En vertu de la proposition 4.12, il existe une constante $c(m, \bar{E}, k)$ telle que

$$
\forall x \in \bigwedge^{m} E, \quad h_{\wedge^{m} \bar{E}}(x) \geq c(m, \bar{E}, k) ;
$$

de la sorte on obtient la majoration $\widehat{\mu}_{\mathrm{n}}(\bar{F}) \leq-c(m, \bar{E}, k) / m$ et la constante $c(\bar{E}, k):=\max \{-c(m, \bar{E}, k) D / m ; 1 \leq m \leq \operatorname{dim} E\}$ convient dans ce cas. En réalité, si $a \leq \widehat{\mu}_{\mathrm{n}}(\bar{F})$ alors la hauteur de $f_{1} \wedge \cdots \wedge f_{m}$ est majorée et la proposition 4.12 entraîne la finitude du nombre de sous-espaces $F$ possibles. Si $\bar{E}$ est un fibré vectoriel adélique quelconque, on utilise les métriques de Löwner, qui satisfont aux inégalités $|\cdot|_{L(\bar{E}), v} \leq\|\cdot\|_{E, v}$ en toute place $v$ de $k$, pour obtenir $\widehat{\operatorname{deg}}_{\mathrm{n}}(\bar{F}) \leq \widehat{\operatorname{deg}}_{\mathrm{n}}\left(F,|\cdot|_{L(\bar{E})}\right)$. La conclusion découle alors du cas hermitien.

Cela justifie la définition suivante.

DÉFInITION 5.4. La pente maximale d'un fibré vectoriel adélique $\bar{E}$, notée $\widehat{\mu}_{\max }(\bar{E})$, est le nombre réel

$$
\widehat{\mu}_{\max }(\bar{E}):=\max \left\{\widehat{\mu}_{\mathrm{n}}(\bar{F}) ; \bar{F} \subseteq \bar{E}\right\} .
$$

On observe que si $\bar{E}_{1} \preceq \bar{E}_{2}$ alors $\widehat{\mu}_{\text {max }}\left(\bar{E}_{2}\right) \leq \widehat{\mu}_{\max }\left(\bar{E}_{1}\right)$. La première propriété de la pente normalisée donnée à la suite de la définition 5.1 entraîne

$$
-\log (1+\varepsilon)-\frac{1}{D} \log \Delta(\bar{E}) \leq \widehat{\mu}_{\max }(\bar{E})-\widehat{\mu}_{\max }\left(\bar{E}_{\varepsilon}\right) \leq 0,
$$

pour tout fibré vectoriel adélique $\bar{E}$ et tout $\varepsilon>0$. 
Remarque 5.5. De la même manière, on peut considérer la pente minimale d'un fibré vectoriel adélique $\bar{E}$ définie par $\widehat{\mu}_{\min }(\bar{E}):=$ $:=\min \left\{\widehat{\mu}_{\mathrm{n}}(\overline{E / F})\right\}$ où $F$ parcourt les sous-espaces vectoriels stricts de $E$. Il s'agit bien d'un minimum car il n'y a qu'un nombre fini de quotients $E / F$ de pentes plus petites qu'un nombre réel donné (on se ramène à la pente maximale via la proposition 4.22). On dispose aussi de l'encadrement

$$
-\log (1+\varepsilon)-\frac{1}{D} \log \Delta(\bar{E}) \leq \widehat{\mu}_{\min }(\bar{E})-\widehat{\mu}_{\min }\left(\bar{E}_{\varepsilon}\right) \leq 0 .
$$

La dualité marque le lien avec la pente maximale.

Lemme 5.6. Soit $\bar{E}$ un fibré vectoriel adélique sur Spec $k$. On a

$$
\left|\widehat{\mu}_{\max }\left(\overline{E^{\vee}}\right)+\widehat{\mu}_{\min }(\bar{E})\right| \leq \frac{1}{D} \log \Delta(\bar{E}) .
$$

DÉmonstration. Supposons dans un premier temps que $\bar{E}$ est hermitien. Soit $\bar{F}$ un sous-fibré vectoriel strict de $\bar{E}$. L'espace vectoriel dual $(E / F)^{\mathrm{v}}$ s'injecte dans $E^{\mathrm{v}}$. La définition de la pente maximale de $\overline{E^{\mathrm{v}}}$ et les propositions 4.21 et 4.22 dans le cas hermitien donnent

$$
\operatorname{dim}(E / F) \widehat{\mu}_{\max }\left(\overline{E^{\mathrm{v}}}\right) \geq \widehat{\operatorname{deg}}_{\mathrm{n}} \overline{(E / F)^{\mathrm{v}}}=-\widehat{\operatorname{deg}}_{\mathrm{n}} \overline{E / F}
$$

puis $\widehat{\mu}_{\max }\left(\overline{E^{\vee}}\right)+\widehat{\mu}_{\mathrm{n}}(\overline{E / F}) \geq 0$ et donc $\widehat{\mu}_{\max }\left(\overline{E^{\vee}}\right)+\widehat{\mu}_{\text {min }}(\bar{E}) \geq 0$. On montre de la même manière l'inégalité en sens inverse. On a donc $\widehat{\mu}_{\max }\left(\overline{E^{\vee}}\right)=-\widehat{\mu}_{\min }(\bar{E})$ si $\bar{E}$ est hermitien. Dans le cas général, on utilise le fibré vectoriel $\bar{E}_{\varepsilon}$ associé à $\bar{E}$. Compte tenu du fait que la dualité renverse les inégalités pour les normes :

$$
\forall G \subseteq E^{\vee}, \quad\left(G,\left(\left.\mathrm{~d}\left(E \otimes_{k} k_{v}, \ell_{n, k_{v}}^{2}\right)^{-1}(1+\varepsilon)^{-1}|\cdot|\right|_{\varepsilon, v} ^{\vee}\right)_{v}\right) \preceq\left(G,\|\cdot\|_{E}^{\vee}\right) \preceq\left(G,|\cdot|_{\varepsilon}^{\vee}\right),
$$

on a

$$
0 \leq \widehat{\mu}_{\max }\left(\overline{E^{\mathrm{v}}}\right)-\widehat{\mu}_{\max }\left(\overline{E_{\varepsilon}^{\mathrm{v}}}\right) \leq \frac{1}{D} \log 4(\bar{E})+\log (1+\varepsilon) .
$$

On somme avec l'estimation du même type entre pentes minimales qui précède l'énoncé du lemme 5.6, puis l'on fait tendre $\varepsilon$ vers 0 et le lemme s'en déduit.

PropriÉtés 5.7. Soit $\bar{E}, \bar{F}$ des fibrés vectoriels adéliques $\operatorname{sur} \operatorname{Spec} k$.

1) Si $\operatorname{dim} F=1$ alors, pour toute norme tensorielle adélique $\alpha$ d'ordre 2 , on a

$$
\widehat{\mu}_{\max }\left(\bar{E} \otimes_{\alpha} \bar{F}\right)=\widehat{\mu}_{\max }(\bar{E})+\widehat{\operatorname{deg}}_{\mathrm{n}} \bar{F} .
$$


2) Pour la somme directe hermitienne $\bar{E} \oplus_{2} \bar{F}$, on a

$$
0 \leq \widehat{\mu}_{\max }\left(\bar{E} \oplus_{2} \bar{F}\right)-\max \left\{\widehat{\mu}_{\max }(\bar{E}), \widehat{\mu}_{\max }(\bar{F})\right\} \leq \frac{1}{D} \log \max \{\Delta(\bar{E}), \Delta(\bar{F})\} .
$$

3) Dans le cas général, on a

$$
0 \leq \widehat{\mu}_{\max }\left(\bar{E} \oplus_{\varsigma} \bar{F}\right)-\max \left\{\widehat{\mu}_{\max }(\bar{E}), \widehat{\mu}_{\max }(\bar{F})\right\} \leq \frac{1}{D} \log (\sqrt{2} \max \{\Delta(\bar{E}), \Delta(\bar{F})\})
$$

(la somme directe $\bar{E} \oplus_{\varsigma} \bar{F}$ est celle définie au paragraphe 3.3).

DÉmonstration. 1) Soit $\overline{E^{\prime}}$ un sous-fibré vectoriel adélique de $\bar{E}$. D'après la proposition 5.2, la pente normalisée de $\overline{E^{\prime}} \otimes_{\alpha} \bar{F}$ est la somme de $\widehat{\mu}_{\mathrm{n}}\left(\overline{E^{\prime}}\right)$ et de $\widehat{\mu}_{\mathrm{n}}(\bar{F})=\widehat{\operatorname{deg}}_{\mathrm{n}} \bar{F}$, car $F$ est une droite. De plus, d'après la proposition 2.12 , on a $\left(E^{\prime} \otimes F, \alpha(\cdot ; \bar{E}, \bar{F})\right) \preceq \overline{E^{\prime}} \otimes_{\alpha} \bar{F}$ et le lemme 4.2 entraîne

$$
\widehat{\mu}_{\mathrm{n}}\left(\overline{E^{\prime}} \otimes_{\alpha} \bar{F}\right) \leq \widehat{\mu}_{\max }\left(\bar{E} \otimes_{\alpha} \bar{F}\right) .
$$

On a donc $\widehat{\mu}_{\mathrm{n}}\left(\overline{E^{\prime}}\right) \leq \widehat{\mu}_{\max }\left(\bar{E} \otimes_{\alpha} \bar{F}\right)-\widehat{\operatorname{deg}}_{\mathrm{n}} \bar{F}$ et, en faisant varier $\overline{E^{\prime}}$, on obtient

$$
\widehat{\mu}_{\max }(\bar{E}) \leq \widehat{\mu}_{\max }\left(\bar{E} \otimes_{\alpha} \bar{F}\right)-\widehat{\operatorname{deg}}_{\mathrm{n}} \bar{F} .
$$

Si l'on applique maintenant cette majoration à $\bar{E} \otimes_{\alpha} \bar{F}$ et $\overline{F^{\mathrm{v}}}$ au lieu, respectivement, de $\bar{E}$ et $\bar{F}$, on obtient l'inégalité en sens inverse, grâce à la propriété $\widehat{\operatorname{deg}}_{\mathrm{n}} \overline{F^{\mathrm{v}}}=-\widehat{\operatorname{deg}}_{\mathrm{n}} \bar{F}$, valide car $\bar{F}$ est automatiquement hermitien, et car $\left(\bar{E} \otimes_{\alpha} \bar{F}\right) \otimes_{\alpha} \overline{F^{v}}$ est isomorphe isométriquement à $\bar{E}$.

2) La positivité de la différence des pentes maximales est immédiate en notant qu'un sous-espace vectoriel de $E$ ou $F$ est aussi un sous-espace de $E \oplus F$, avec compatibilité des normes. Ceci s'applique également pour $\bar{E} \oplus_{\varsigma} \bar{F}$ puisque la norme $\|\cdot\|_{\bar{E} \oplus_{\varsigma} \bar{F}, v}$ restreinte à $E$ ou $F$ est celle qui est naturellement sur ces espaces.

Pour la majoration, supposons dans un premier temps que $\bar{E}$ et $\bar{F}$ sont hermitiens. Soit $\bar{H}$ un sous-fibré vectoriel adélique de $\bar{E} \oplus_{2} \bar{F}$. Posons $H_{1}:=H \cap E$. Par définition des métriques quotient, on a $\overline{H / H_{1}}=\bar{H} / \overline{H_{1}}$ et donc, puisque ces fibrés adéliques sont hermitiens,

$$
\widehat{\operatorname{deg}}_{\mathrm{n}} \bar{H}=\widehat{\operatorname{deg}}_{\mathrm{n}} \bar{H}_{1}+\widehat{\operatorname{deg}}_{\mathrm{n}}\left(\overline{H / H_{1}}\right) .
$$

Le premier terme $\widehat{\operatorname{deg}}_{\mathrm{n}} \bar{H}_{1}$ est naturellement inférieur à $\left(\operatorname{dim} H_{1}\right) \widehat{\mu}_{\max }(\bar{E})$. Pour le second terme, considérons l'injection $l: H / H_{1} \hookrightarrow F$, $\imath\left(x \oplus y \bmod H_{1}\right)=y$. L'application $\imath$ n'est pas nécessairement une isométrie en toute place $v$ de $k$ (et done $\overline{H / H_{1}}$ n'est pas en général un sous-fibré 
adélique de $\bar{F}$ ). Néanmoins, comme $\|x \oplus y\|_{E \oplus_{2} F, v} \geq\|y\|_{F, v}$ pour tout $x \oplus y \in H_{v}$, on a

$$
\widehat{\operatorname{deg}}_{\mathrm{n}}\left(\overline{H / H_{1}}\right) \leq \widehat{\operatorname{deg}}_{\mathrm{n}}\left(\imath\left(H / H_{1}\right),\|\cdot\|_{F}\right) \leq\left(\operatorname{dim} H / H_{1}\right) \widehat{\mu}_{\max }(\bar{F}) .
$$

On en déduit

$$
\widehat{\mu}_{\mathrm{n}}(\bar{H})=\frac{\widehat{\operatorname{deg}}_{\mathrm{n}}(\bar{H})}{\operatorname{dim} H} \leq \frac{\left(\operatorname{dim} H_{1}\right) \widehat{\mu}_{\max }(\bar{E})+\left(\operatorname{dim} H / H_{1}\right) \widehat{\mu}_{\max }(\bar{F})}{\operatorname{dim} H}
$$

puis $\widehat{\mu}_{\max }\left(\bar{E} \oplus_{2} \bar{F}\right) \leq \max \left\{\widehat{\mu}_{\max }(\bar{E}), \widehat{\mu}_{\max }(\bar{F})\right\}$, et avec la remarque du début de ce point 2 ), on obtient l'égalité souhaitée dans le cas hermitien.

Revenons maintenant au cas général de deux fibrés vectoriels adéliques quelconques. Soit $\varepsilon>0$ et $\bar{E}_{\varepsilon}=\left(E,|\cdot|_{\varepsilon, E, v}\right)\left(\operatorname{resp} . \bar{F}_{\varepsilon}=\left(F,|\cdot|_{\varepsilon, F, v}\right)\right)$ un fibré adélique hermitien associé à $\bar{E}$ (resp. $\bar{F}$ ), comme celui introduit à la suite de (28). Par définition, pour tous $x \in E_{v}, y \in F_{v}$, on a $|x|_{\varepsilon, E, v}^{2}+|y|_{\varepsilon, F, v}^{2} \leq$ $\leq\|(x, y)\|_{\bar{E} \oplus_{2} \bar{F}, v}^{2}$ et donc

$$
\widehat{\mu}_{\max }\left(\bar{E} \oplus_{2} \bar{F}\right) \leq \widehat{\mu}_{\max }\left(\bar{E}_{\varepsilon} \oplus_{2} \bar{F}_{\varepsilon}\right)=\max \left\{\widehat{\mu}_{\max }\left(\bar{E}_{\varepsilon}\right), \widehat{\mu}_{\max }\left(\bar{F}_{\varepsilon}\right)\right\} .
$$

La pente maximale de $\bar{E}_{\varepsilon}$ est à son tour plus petite que $\widehat{\mu}_{\max }(\bar{E})+$ $+\frac{1}{D} \log \Delta(\bar{E})+\log (1+\varepsilon)$. Le même type de majoration pour $\widehat{\mu}_{\max }\left(\bar{F}_{\varepsilon}\right)$ puis $\varepsilon \rightarrow 0$ donnent l'inégalité souhaitée.

3) En définissant $\varsigma$, on avait observé que $\|\cdot\|_{\bar{E} \oplus_{\infty} \bar{F}} \leq\|\cdot\|_{\bar{E} \oplus_{\zeta} \bar{F}}$. Comme $(\sqrt{2})^{-1}\|\cdot\|_{\bar{E}_{\oplus_{2}} \bar{F}} \leq\|\cdot\|_{\bar{E} \oplus_{\infty} \bar{F}}$, le problème se ramène à majorer la pente maximale de $\bar{E} \oplus_{2} \bar{F}$, ce que nous venons de faire.

Remarque 5.8. Soit $N \in \mathbf{N} \backslash\{0\}$ et $\overline{E_{1}}, \ldots, \overline{E_{N}}$ des fibrés vectoriels adéliques sur Spec $k$. Alors on a

$$
0 \leq \widehat{\mu}_{\max }\left(\overline{E_{1}} \oplus_{2} \cdots \oplus_{2} \bar{E}_{N}\right)-\max _{1 \leq i \leq N}\left\{\widehat{\mu}_{\max }\left(\overline{E_{i}}\right)\right\} \leq \frac{1}{D} \log \max _{1 \leq i \leq N}\left\{\Delta\left(\overline{E_{i}}\right)\right\} .
$$

Et, pour une somme directe plus générale comme dans le point 3) des propriétés 5.7 , on doit rajouter un $\sqrt{N}$ devant le maximum des $\Delta\left(\overline{E_{i}}\right)$. Ces généralisations se démontrent en approchant chacun des fibrés vecto-

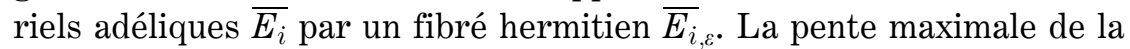
somme directe hermitienne des $\overline{E_{i, \varepsilon}}$ est le maximum de chacune des pentes maximales $\widehat{\mu}_{\max }\left(\bar{E}_{i, \varepsilon}\right)$ et on procède alors comme dans la démonstration ci-dessus. Le nombre $\sqrt{N}$ provient de la comparaison entre les normes de $\ell_{N}^{2}$ et $\ell_{N}^{\infty}$.

$\mathrm{Au} \S 7$, nous obtiendrons également une estimation de la pente maximale de la $\ell^{\text {ème }}$ puissance symétrique $S^{\ell}(\bar{E})$. 
5.2. - Autres pentes.

Soit $\bar{E}$ un fibré vectoriel adélique sur $\operatorname{Spec} k$, de dimension $n$. La proposition 5.3 implique que l'intersection de l'ensemble $\left\{\left(\operatorname{dim} F, \widehat{\operatorname{deg}}_{n} \bar{F}\right) ; \bar{F} \subseteq \bar{E}\right\}$ avec tout demi-plan supérieur est fini. L'enveloppe convexe de cet ensemble est délimitée par une fonction affine par morceaux et concave $P_{\bar{E}}:[0, n] \rightarrow \mathbf{R}$. Les sommets du graphe de $P_{\bar{E}}$ sont atteints par des sousfibrés adéliques de $\bar{E}$. On a $P_{\bar{E}}(0)=0$ et $P_{\bar{E}}(n)=\widehat{\operatorname{deg}}_{n} \bar{E}$. On note que si $\bar{F} \subseteq \bar{E}$ alors, pour tout $x \in[0, \operatorname{dim} F]$, on a $P_{\bar{F}}(x) \leq P_{\bar{E}}(x)$.

Définition 5.9. Pour $i \in\{1, \ldots, n\}$, la $i^{\text {ème }}$ pente normalisée de $\bar{E}$, notée $\widehat{\mu}_{i}(\bar{E})$, est le nombre réel

$$
\widehat{\mu}_{i}(\bar{E}):=P_{\bar{E}}(i)-P_{\bar{E}}(i-1) .
$$

De la sorte, on a

$$
\widehat{\operatorname{deg}}_{\mathrm{n}} \bar{E}=\sum_{i=1}^{n} \widehat{\mu}_{i}(\bar{E}) .
$$

Soit $\varepsilon>0$. Compte tenu de l'encadrement des normes de $\bar{E}$ en fonction de celles du fibré adélique hermitien $\bar{E}_{\varepsilon}$ (voir (28)), on a

$$
\forall x \in[0, n], \quad P_{\bar{E}_{\varepsilon}}(x)-\frac{x}{D}(\log \Delta(\bar{E})+D \log (1+\varepsilon)) \leq P_{\bar{E}}(x) \leq P_{\bar{E}_{\varepsilon}}(x),
$$

ce qui implique

$$
\begin{aligned}
& -\frac{i}{D}(\log \Delta(\bar{E})+D \log (1+\varepsilon)) \\
& \quad \leq \widehat{\mu}_{i}(\bar{E})-\widehat{\mu}_{i}\left(\bar{E}_{\varepsilon}\right) \leq \frac{i-1}{D}(\log \Delta(\bar{E})+D \log (1+\varepsilon)) .
\end{aligned}
$$

Cet encadrement se révèle particulièrement précieux pour généraliser les propriétés des pentes d'un fibré adélique hermitien à un fibré vectoriel adélique quelconque.

Par ailleurs, la concavité de $P_{\bar{E}}$ entraîne la décroissance de la suite $\left(\widehat{\mu}_{i}(\bar{E})\right)_{1 \leq i \leq n}$.

LEMME 5.10. On $a$

$$
\widehat{\mu}_{1}(\bar{E})=\widehat{\mu}_{\max }(\bar{E}) \text {. }
$$

DÉmonstration. Pour tout sous-fibré $\bar{F}$ de $\bar{E}$ de dimension $m$, on a

$$
\widehat{\operatorname{deg}}_{\mathrm{n}} \bar{F} \leq P_{\bar{E}}(m)=\sum_{i=1}^{m} \widehat{\mu}_{i}(\bar{E}) \leq m \widehat{\mu}_{1}(\bar{E})
$$


donc $\widehat{\mu}_{\mathrm{n}}(\bar{F}) \leq \widehat{\mu}_{1}(\bar{E})$, puis $\widehat{\mu}_{\max }(\bar{E}) \leq \widehat{\mu}_{1}(\bar{E})$. Inversement, si l'on choisit pour $\bar{F}$ le premier sommet non trivial de $P_{\bar{E}}$, on a $\widehat{\mu}_{1}(\bar{E})=\widehat{\mu}_{\mathrm{n}}(\bar{F})$ et donc $\widehat{\mu}_{1}(\bar{E}) \leq \widehat{\mu}_{\max }(\bar{E})$.

Je ne sais pas si l'égalité $\widehat{\mu}_{n}(\bar{E})=\widehat{\mu}_{\text {min }}(\bar{E})$ est vraie en général. Cependant on a le résultat suivant qui assure l'égalité dans le cas d'un fibré vectoriel hermitien.

Lemme 5.11. Soit $\bar{E}$ un fibré vectoriel adélique sur Spec $k$, de dimension $n \geq 1$. On $a$

$$
\left|\widehat{\mu}_{n}(\bar{E})-\widehat{\mu}_{\min }(\bar{E})\right| \leq \frac{n}{D} \log \Delta(\bar{E}) .
$$

Il faut prendre garde à ne pas confondre dans cette inégalité la $n^{\text {ème }}$ pente $\widehat{\mu}_{n}(\bar{E})$ de $\bar{E}$ avec sa pente normalisée $\widehat{\mu}_{\mathrm{n}}(\bar{E})$.

DÉmonstration. Lorsque $\bar{E}$ est hermitien, nous montrerons un peu plus loin que $\widehat{\mu}_{n}(\bar{E})=-\widehat{\mu}_{1}\left(\overline{E^{v}}\right)$ (voir propriété (5.14), (2)). Dans ce cas, l'égalité $\widehat{\mu}_{n}(\bar{E})=\widehat{\mu}_{\min }(\bar{E})$ résulte des lemmes 5.6 et 5.10. Le cas d'un fibré vectoriel adélique quelconque s'en déduit au moyen du fibré hermitien $\bar{E}_{\varepsilon}$, de l'encadrement (36) et de la remarque 5.5.

Filtration canonique dans le cas hermitien.

Lorsque $\bar{E}$ est un fibré adélique hermitien, les sous-fibrés de $\bar{E}$ qui correspondent aux sommets du graphe de $P_{\bar{E}}$ sont uniques et ils forment une filtration de $\bar{E}$.

Lemme 5.12. Soit $\bar{E}$ un fibré adélique hermitien et $i \in\{1, \ldots, n-1\}$ un point de non-dérivabilité de $P_{\bar{E}}$. Alors il existe un unique sous-espace vectoriel $E_{i}$ de $E$, de dimension $i$, tel que $\widehat{\operatorname{deg}}_{\mathrm{n}} \overline{E_{i}}=P_{\bar{E}}(i)$.

DÉmonstration. L'existence de $E_{i}$ est une conséquence du fait qu'il n'y a qu'un nombre fini de sous-espaces de $E$ dont le degré adélique est plus grand qu'une constante. Pour montrer l'unicité, on raisonne par l'absurde et on suppose qu'il existe $E_{i}$ et $E_{i}^{\prime}$ deux sous-espaces vectoriels distincts de $E$, de même dimension $i$, et de degrés adéliques égaux à $P_{\bar{E}}(i)$. Soit $\delta_{1}:=\operatorname{dim}\left(E_{i}+E_{i}^{\prime}\right)$ et $\delta_{2}:=\operatorname{dim}\left(E_{i} \cap E_{i}^{\prime}\right)$. Par concavité de $P_{\bar{E}}$ et comme $\delta_{1} \neq i$, on a

$$
\frac{P_{\bar{E}}\left(\delta_{1}\right)-P_{\bar{E}}(i)}{\delta_{1}-i}<\frac{P_{\bar{E}}(i)-P_{\bar{E}}\left(\delta_{2}\right)}{i-\delta_{2}}
$$


et donc, compte tenu de la relation $\delta_{1}-i=i-\delta_{2}$, on a

$$
\widehat{\operatorname{deg}}_{\mathrm{n}} \overline{E_{i}+E_{i}^{\prime}}+\widehat{\operatorname{deg}}_{\mathrm{n}} \overline{E_{i} \cap E_{i}^{\prime}} \leq P_{\bar{E}}\left(\delta_{1}\right)+P_{\bar{E}}\left(\delta_{2}\right)<2 P_{\bar{E}}(i) .
$$

D'après la proposition 4.23 et comme $\bar{E}$ est hermitien, le membre de gauche de (37) est minoré par la somme $\widehat{\operatorname{deg}}_{\mathrm{n}} \overline{E_{i}}+\widehat{\operatorname{deg}}_{\mathrm{n}} \overline{E_{i}^{\prime}}=2 P_{\bar{E}}(i)$ et ceci contredit (37).

Proposition 5.13. Soit $\bar{E}$ un fibré adélique hermitien sur Spec $k$. Il existe une unique filtration $\{0\}=E_{0} \subsetneq E_{1} \subsetneq \cdots \subsetneq E_{g}:=E$ telle que, pour tout $j \in\{0, \ldots, g\}$, on ait $P_{\bar{E}}\left(\operatorname{dim} E_{j}\right)=\overline{\operatorname{deg}}_{\mathrm{n}} \overline{E_{j}}$ et $\left\{\operatorname{dim} E_{j} ; 1 \leq j \leq g-1\right\}$ est l'ensemble des points de non-dérivabilité de la fonction $P_{\bar{E}}$.

Cette filtration est appelée filtration canonique, ou filtration d'Harder-Narasimhan-Stuhler-Grayson.

DÉmonstration. Le lemme 5.12 montre l'existence et l'unicité des $\overline{E_{j}}$. Il ne reste plus qu'à prouver l'inclusion $E_{j} \subseteq E_{j+1}$. Elle repose exactement sur le même argument que celui employé dans la preuve du lemme 5.12, appliqué aux sous-espaces $E_{j}, E_{j+1}, E_{j} \cap E_{j+1}$ et $E_{j}+E_{j+1}$.

La démonstration donnée ici de l'existence et de l'unicité de la filtration canonique dans le cas hermitien s'adapte mal au cas général en partie à cause de la minoration du degré d'une somme de sous-espaces de $E$, qui fait intervenir la distance adélique à l'espace hermitien $\left(k^{n},|\cdot|_{2}\right)$. Cela empêche les arguments de concavité de $P_{\bar{E}}$ de fonctionner.

PropriÉTÉs 5.14. Soit $\bar{L}$ un fibré en droites adélique et $\bar{E}$ un fibré vectoriel adélique.

1) Pour toute norme tensorielle adélique $\alpha$ d'ordre 2 et pour tout $x \in[0, n]$, on a

$$
P_{\bar{E} \otimes_{\alpha} \bar{L}}(x)=P_{\bar{E}}(x)+x \widehat{\operatorname{deg}}_{\mathrm{n}} \bar{L} .
$$

En particulier, pour tout $i \in\{1, \ldots, n\}$, on a

$$
\widehat{\mu}_{i}\left(\bar{E} \otimes_{\alpha} \bar{L}\right)=\widehat{\mu}_{i}(\bar{E})+\widehat{\operatorname{deg}}_{\mathrm{n}} \bar{L} .
$$

2) Pour tout $i \in\{1, \ldots, n\}$, on a

$$
\left|\widehat{\mu}_{i}\left(\overline{E^{\vee}}\right)+\widehat{\mu}_{n-i+1}(\bar{E})\right| \leq \widehat{\operatorname{deg}}_{\mathrm{n}} L(\bar{E})-\widehat{\operatorname{deg}}_{\mathrm{n}} J(\bar{E})+\frac{n}{D} \log \Delta(\bar{E}) .
$$


3) Pour tout $i \in\{1, \ldots, n\}$, on a

$$
\left|\widehat{\mu}_{i}(\bar{E})-\min _{E_{2}} \max _{E_{1}}\left\{\widehat{\mu}_{\mathrm{n}}\left(\overline{E_{1} / E_{2}}\right)\right\}\right| \leq \frac{i}{D} \log \Delta(\bar{E})
$$

où $E_{1}$ et $E_{2}$ varient parmi les sous-espaces vectoriels de $E$ vérifiant $E_{2} \subseteq E_{1}, \operatorname{dim} E_{1} \geq i$ et $\operatorname{dim} E_{2} \leq i-1$. On a aussi

$$
\left|\widehat{\mu}_{i}(\bar{E})-\max _{E_{1}} \min _{E_{2}}\left\{\widehat{\mu}_{\mathrm{n}}\left(\overline{E_{1} / E_{2}}\right)\right\}\right| \leq \frac{i}{D} \log \Delta(\bar{E}) .
$$

Remarque 5.15. La différence $\widehat{\operatorname{deg}}_{\mathrm{n}} L(\bar{E})-\widehat{\operatorname{deg}}_{\mathrm{n}} J(\bar{E})$ qui est dans l'estimation 2) est plus petite que $\frac{n}{D} \log \Delta(\bar{E})$ (voir (29)), terme lui-même inférieur à $n \log (2 n)$ (voir (27)).

DÉmonstration. 1) Cela résulte directement de la formule $\widehat{\operatorname{deg}}_{\mathrm{n}}\left(\bar{F} \otimes_{\alpha} \bar{L}\right)=\widehat{\operatorname{deg}}_{\mathrm{n}} \bar{F}+(\operatorname{dim} F) \widehat{\operatorname{deg}}_{\mathrm{n}} \bar{L}$ qui découle de la proposition 5.2.

2) Pour tout sous-fibré adélique $\imath: \bar{F} \hookrightarrow \bar{E}$ de dimension $m$, on a une suite exacte $0 \rightarrow \operatorname{ker} l^{\mathrm{v}} \rightarrow E^{\mathrm{v}} \rightarrow F^{\mathrm{v}} \rightarrow 0$ et done (preuve de la proposition 4.22)

$$
\left|\widehat{\operatorname{deg}}_{\mathrm{n}} \overline{E^{\mathrm{v}}}-\widehat{\operatorname{deg}}_{\mathrm{n}} \overline{F^{\mathrm{v}}}-\widehat{\operatorname{deg}}_{\mathrm{n}} \overline{\operatorname{ker} l^{v}}\right| \leq \widehat{\operatorname{deg}}_{\mathrm{n}} L\left(\overline{E^{\mathrm{v}}}\right)-\widehat{\operatorname{deg}}_{\mathrm{n}} J\left(\overline{E^{\mathrm{v}}}\right),
$$

ce qui entraîne

$$
\widehat{\operatorname{deg}}_{\mathrm{n}} \bar{F} \leq \widehat{\operatorname{deg}}_{\mathrm{n}} \overline{\operatorname{ker} l^{\mathrm{v}}}+\widehat{\operatorname{deg}}_{\mathrm{n}} L\left(\overline{E^{\mathrm{v}}}\right)-\widehat{\operatorname{deg}}_{\mathrm{n}} J\left(\overline{E^{\mathrm{v}}}\right)-\widehat{\operatorname{deg}}_{\mathrm{n}} \overline{E^{\mathrm{v}}}
$$

grâce à la proposition 4.21. Le degré adélique normalisé de $\overline{\operatorname{ker} l^{v}}$ est majoré par $P_{\overline{E^{\vee}}}(n-m)$. De plus, la dualité $J\left(\overline{E^{\vee}}\right)=L(\bar{E})^{\vee}$ entre les fibrés hermitiens de John et Löwner donne

$$
\widehat{\operatorname{deg}}_{\mathrm{n}} L\left(\overline{E^{\mathrm{v}}}\right)-\widehat{\operatorname{deg}}_{\mathrm{n}} J\left(\overline{E^{\mathrm{v}}}\right)=\widehat{\operatorname{deg}}_{\mathrm{n}} L(\bar{E})-\widehat{\operatorname{deg}}_{\mathrm{n}} J(\bar{E}) .
$$

On en déduit

$$
P_{\bar{E}}(m) \leq P_{\overline{E^{\mathrm{v}}}}(n-m)+\widehat{\operatorname{deg}}_{\mathrm{n}} L(\bar{E})-\widehat{\operatorname{deg}}_{\mathrm{n}} J(\bar{E})-\widehat{\operatorname{deg}}_{\mathrm{n}} \overline{E^{\mathrm{v}}} .
$$

En échangeant les rôles de $\bar{E}$ et de son dual, on a aussi la majoration

$$
P_{\overline{E^{\mathrm{v}}}}(m) \leq P_{\bar{E}}(n-m)+\widehat{\operatorname{deg}}_{\mathrm{n}} L(\bar{E})-\widehat{\operatorname{deg}}_{\mathrm{n}} J(\bar{E})-\widehat{\operatorname{deg}}_{\mathrm{n}} \bar{E} .
$$

Par définition des pentes $\widehat{\mu}_{i}$, on a alors

$$
\left|\widehat{\mu}_{i}\left(\overline{E^{\mathrm{v}}}\right)+\widehat{\mu}_{n-i+1}(\bar{E})\right| \leq 2\left(\widehat{\operatorname{deg}}_{\mathrm{n}} L(\bar{E})-\widehat{\operatorname{deg}}_{\mathrm{n}} J(\bar{E})\right)-\left(\widehat{\operatorname{deg}}_{\mathrm{n}} \bar{E}+\widehat{\operatorname{deg}}_{\mathrm{n}} \overline{E^{\mathrm{v}}}\right) .
$$

$\mathrm{Au}$ moyen de la proposition 4.9 et de l'inégalité $\widetilde{\operatorname{vr}}(\bar{E}) \widetilde{\operatorname{vr}}\left(\overline{E^{\mathrm{v}}}\right) \leq \Delta(\bar{E})$, ana- 
logue à (27), l'on déduit

$$
\begin{aligned}
& \widehat{\operatorname{deg}}_{\mathrm{n}} L(\bar{E})-\widehat{\operatorname{deg}}_{\mathrm{n}} J(\bar{E})-\left(\widehat{\operatorname{deg}}_{\mathrm{n}} \bar{E}+\widehat{\operatorname{deg}}_{\mathrm{n}} \overline{E^{\mathrm{v}}}\right) \\
& =\widehat{\operatorname{deg}}_{\mathrm{n}} L(\bar{E})+\widehat{\operatorname{deg}}_{\mathrm{n}} L\left(\overline{E^{\mathrm{v}}}\right)-\left(\widehat{\operatorname{deg}}_{\mathrm{n}} \bar{E}+\widehat{\operatorname{deg}}_{\mathrm{n}} \overline{E^{\mathrm{v}}}\right) \leq \frac{n}{D} \log \Delta(\bar{E}) .
\end{aligned}
$$

On notera au passage l'égalité $P_{\bar{E}}(m)=P_{\overline{E^{\mathrm{v}}}}(n-m)+\widehat{\operatorname{deg}}_{\mathrm{n}} \bar{E}$ obtenue lorsque $\bar{E}$ est hermitien.

3) On commence par établir l'égalité

$$
\widehat{\mu}_{i}(\bar{E})=\min _{E_{2}} \max _{E_{1}}\left\{\widehat{\mu}_{\mathrm{n}}\left(\overline{E_{1} / E_{2}}\right)\right\}
$$

lorsque $\bar{E}$ est un fibré adélique hermitien. On note $\alpha$ le minimax du membre de droite. Soit $\overline{E_{2}} \subseteq \overline{E_{1}}$ de dimensions respectives $n_{2}$ et $n_{1}$. On suppose $n_{2} \leq i-1<n_{1}$. D'après la proposition 4.22 , on a

$$
\widehat{\mu}_{\mathrm{n}}\left(\overline{E_{1} / E_{2}}\right) \leq \frac{P_{\bar{E}}\left(n_{1}\right)-\widehat{\operatorname{deg}}_{\mathrm{n}} \overline{E_{2}}}{n_{1}-n_{2}}
$$

et donc $\widehat{\operatorname{deg}}_{n} \overline{E_{2}} \leq P_{\bar{E}}\left(n_{1}\right)-\left(n_{1}-n_{2}\right) \alpha$ puis $P_{\bar{E}}\left(n_{2}\right) \leq P_{\bar{E}}\left(n_{1}\right)-\left(n_{1}-n_{2}\right) \alpha$. Inversement, on a

$$
\frac{\widehat{\operatorname{deg}}_{\mathrm{n}} \overline{E_{1}}-P_{\bar{E}}\left(n_{2}\right)}{n_{1}-n_{2}} \leq \widehat{\mu}_{\mathrm{n}}\left(\overline{E_{1} / E_{2}}\right) \leq \max _{E_{1}}\left\{\widehat{\mu}_{\mathrm{n}}\left(\overline{E_{1} / E_{2}}\right)\right\}
$$

et donc $P_{\bar{E}}\left(n_{1}\right) \leq P_{\bar{E}}\left(n_{2}\right)+\left(n_{1}-n_{2}\right) \alpha$. Ainsi, pour tout $n_{2}<i \leq n_{1}$, on a

$$
P_{\bar{E}}\left(n_{1}\right)=P_{\bar{E}}\left(n_{2}\right)+\left(n_{1}-n_{2}\right) \alpha
$$

et donc, par définition, $\alpha=\widehat{\mu}_{i}(\bar{E})$. Le passage au cas général s'effectue au moyen du fibré $\bar{E}_{\varepsilon}$ associé à $\bar{E}$ et pour un nombre réel $\varepsilon>0$ donné (voir (28)). On observe que

$$
\begin{gathered}
-\frac{1}{D}(\log \Delta(\bar{E})+D \log (1+\varepsilon))+\widehat{\mu}_{\mathrm{n}}\left(E_{1} / E_{2},|\cdot|_{\varepsilon, \bar{E}}\right) \\
\leq \widehat{\mu}_{\mathrm{n}}\left(\overline{E_{1} / E_{2}}\right) \leq \widehat{\mu}_{\mathrm{n}}\left(E_{1} / E_{2},|\cdot|_{\varepsilon, \bar{E}}\right)
\end{gathered}
$$

puis, avec la formule (40),

$$
-\frac{1}{D} \log \Delta(\bar{E})-\log (1+\varepsilon)+\widehat{\mu}_{i}\left(\bar{E}_{\varepsilon}\right) \leq \min _{E_{2}} \max _{E_{1}}\left\{\widehat{\mu}_{\mathrm{n}}\left(\overline{E_{1} / E_{2}}\right)\right\} \leq \widehat{\mu}_{i}\left(\bar{E}_{\varepsilon}\right) .
$$

Ce qui entraîne l'encadrement annoncé de $\widehat{\mu}_{i}(\bar{E})$ via l'estimation (36) et en faisant tendre $\varepsilon$ vers 0 . L'inégalité (39) s'obtient de la même manière. 


\section{3. - Fibrés adéliques semi-stables.}

DÉFINITION 5.16. Un fibré vectoriel adélique $\bar{E}$ est dit semi-stable si toutes les pentes $\widehat{\mu}_{i}(\bar{E}), 1 \leq i \leq n$, sont égales.

De manière équivalente, le fibré $\bar{E}$ est semi-stable si $\widehat{\mu}_{1}(\bar{E})$ est la pente normalisée $\widehat{\mu}_{\mathrm{n}}(\bar{E})$ de $\bar{E}$, ou si, pour tout $m \in[0, n], P_{\bar{E}}(m)=m \widehat{\mu}_{\mathrm{n}}(\bar{E})$ ou bien encore si $\widehat{\mu}_{\max }(\bar{E})=\widehat{\mu}_{\mathrm{n}}(\bar{E})$ (car la somme des $\widehat{\mu}_{i}(\bar{E})$ égale $\widehat{\operatorname{deg}}_{\mathrm{n}} \bar{E}=n \widehat{\mu}_{\mathrm{n}}(\bar{E})$ ). En vertu de la première des propriétés 5.14 , le fibré $\bar{E}$ est semi-stable si et seulement si, pour tout fibré en droites adélique $\bar{L}$ sur Spec $k$, le produit tensoriel $\bar{E} \otimes \bar{L}$ est semi-stable. Par ailleurs, comme l'a mentionné J.B. Bost dans [6], l'on dispose de conditions suffisantes pour vérifier la semi-stabilité de $\bar{E}$.

Proposition 5.17. Supposons qu'il existe un sous-groupe G de GL (E) ayant les propriétés suivantes:

(i) tout élément $\varphi \in G$ induit une isométrie de $E_{v}$ en toutes les places $v$ de $k$,

(ii) pour tout sous-espace vectoriel $E^{\prime}$ de $E$, si l'orbite $\left\{\varphi\left(E^{\prime}\right) ; \varphi \in G\right\}$ est finie alors $E^{\prime}=0$ ou $E^{\prime}=E$.

Alors $\bar{E}$ est semi-stable. Lorsque $\bar{E}$ est hermitien, cette même conclusion reste valide en remplaçant l'hypothèse (ii) par la condition plus faible

(ii) $^{\prime}$ si, pour tout $\varphi \in G$, on a $\varphi\left(E^{\prime}\right)=E^{\prime}$ alors $E^{\prime}=0$ ou $E^{\prime}=E$ (l'action de $G$ sur $E$ est irréductible).

DÉmonstration. La condition (i) implique que, pour tout $\overline{E^{\prime}} \subseteq \bar{E}$, on a $\widehat{\operatorname{deg}} \overline{E^{\prime}}=\widehat{\operatorname{deg}} \overline{\varphi\left(E^{\prime}\right)}$. En particulier, si $\overline{E^{\prime}}$ est un sommet du polygone canonique construit avec $P_{\bar{E}}$, il en est de même pour $\overline{\varphi\left(E^{\prime}\right)}$ et l'orbite de $E^{\prime}$ sous l'action de $G$ est finie. L'hypothèse (ii) entraîne alors $E^{\prime}=\{0\}$ ou $\overline{E^{\prime}}=\bar{E}$, ce qui signifie que $\bar{E}$ est semi-stable. Dans le cas hermitien, la proposition 5.13 donne l'unicité des sous-fibrés vectoriels adéliques de $\bar{E}$ qui forment les sommets du graphe de $P_{\bar{E}}$. On a donc $\varphi\left(E^{\prime}\right)=E^{\prime}$ et l'hypothèse (ii)' suffit alors pour conclure à la semi-stabilité de $\bar{E}$.

La géométrie d'Arakelov fournit une famille d'exemples de fibrés vectoriels hermitiens semi-stables, lorsque $k$ est un corps de nombres. En effet, soit $X$ une variété abélienne définie sur $k$, munie d'un fibré en droites $L$ ample et symétrique. Soit $(\mathcal{X}, \overline{\mathcal{L}})$ un modèle de Moret-Bailly de $(X, L)$, au sens du $§ 4.3 .1$ de [5]. Sans entrer dans les détails, rappelons que $\mathcal{X}$ est un 
schéma en groupes semi-stable sur l'anneau des entiers d'une extension finie $K$ de $k$, de fibre générique $A \otimes K$ et $\overline{\mathcal{L}}$ est un fibré hermitien cubiste, de fibre générique $L_{K}$. L'espace des section globales $\mathrm{H}^{0}(\mathcal{X}, \mathcal{L})$ est un $\mathcal{O}_{K^{-}}$ module projectif de type fini. Les métriques sur $\overline{\mathcal{L}}$ le munissent de métriques hermitiennes aux places archimédiennes de $K$, ce qui lui confère alors une structure de fibré adélique hermitien $\overline{\mathrm{H}^{0}(X, L)}$ sur $K$. Au moyen du groupe de Mumford qui agit irréductiblement sur $\mathrm{H}^{0}(X, L)$, J.-B. Bost a montré que $\overline{\mathrm{H}^{0}(X, L)}$ est semi-stable (et on l'on connait même une expression de sa pente normalisée, voir le théorème 4.2 de [6], ainsi que le théorème 2.10 , ii), de [18] et aussi le dernier paragraphe).

\section{4. - Minima successifs adéliques.}

Comme le montre le travail de T. Borek [3] dans le cas des fibrés vectoriels hermitiens sur $\operatorname{Spec} \mathcal{O}_{k}$, les pentes de $\bar{E}$ telles que nous venons de les définir sont étroitement liées aux minima successifs adéliques de $\bar{E}$. Si $a=\left(a_{v}\right)_{v}$ est un élément de $k_{\mathrm{A}}$, on note $\mathrm{B}(\bar{E}, a)$ l'ensemble

$$
\mathrm{B}(\bar{E}, a)=\left\{\left(x_{v}\right)_{v} \in E_{\mathbf{A}} ;\left\|x_{v}\right\|_{E, v} \leq\left|a_{v}\right|_{v} \text { pour toute place } v \text { de } k\right\}
$$

(ainsi $\mathbb{B}(\bar{E}, 1)=\mathbb{B}(\bar{E})$ ).

DÉFINITION 5.18. Soit $\bar{E}$ un fibré vectoriel adélique sur $\operatorname{Spec} k$, de dimension $n$. Pour $i \in\{1, \ldots, n\}$, le $i^{\text {ème }}$ minimum relatif à $\bar{E}$, que l'on notera $\lambda_{i}(\bar{E})$, est la borne inférieure de l'ensemble des nombres réels positifs de la forme $|a|_{\mathrm{A}}$ où $a \in k_{\mathrm{A}}$ est tel que $E \cap \mathbb{B}(\bar{E}, a)$ contienne $i$ vecteurs $k$-linéairement indépendants.

Ces nombres $\lambda_{i}(\bar{E})$ dépendent a priori du corps $k$. Si $k$ est un corps de nombres, il arrive souvent que l'on se restreigne à des éléments $a$ de la forme $(\lambda, \ldots, \lambda, 1, \ldots)$ où $\lambda \in \mathbf{R}^{+}$aux places archimédiennes de $k$, comme le font par exemple E. Bombieri \& J. Vaaler [2]. Les puissances $D^{\text {èmes }}$ de leurs minima sont donc plus grands que ceux considérés ici. La définition donnée ici est empruntée à l'article de J.L. Thunder [38]. On notera que

$$
\lambda_{1}(\bar{E}) \leq \cdots \leq \lambda_{n}(\bar{E})
$$

et que, si $\overline{E^{\prime}}$ est un sous-fibré adélique de $\bar{E}$ alors $\lambda_{i}(\bar{E}) \leq \lambda_{i}\left(\overline{E^{\prime}}\right)$ pour tout $i \in\left\{1, \ldots, \operatorname{dim} E^{\prime}\right\}$. De plus, on a

$$
\lambda_{i}\left(\bar{E}_{\varepsilon}\right) \leq \lambda_{i}(\bar{E}) \leq \Delta(\bar{E})(1+\varepsilon)^{D} \lambda_{i}\left(\bar{E}_{\varepsilon}\right) .
$$

Existe alors une variante du second théorème de Minkowski. 
THÉORÈme 5.19. Le produit des minima successifs de $\bar{E}$ vérifie

$$
\lambda_{1}(\bar{E}) \cdots \lambda_{n}(\bar{E}) \leq \kappa^{n} \frac{\operatorname{covol}(E)}{\operatorname{vol}(\mathbb{B}(\bar{E}))} \operatorname{vr}(\bar{E})^{n}
$$

où $\kappa=2^{[k: \mathbf{Q}]}$ si $k$ est un corps de nombres et $\kappa=q$ si $k$ est un corps de fonctions. Ici, vol désigne une mesure de Haar quelconque sur $E_{\mathrm{A}}$ et $\operatorname{covol}(E)$ est la mesure de l'espace quotient $E_{\mathrm{A}} / E$, relative $\grave{a}$ vol.

Dans le cas d'un corps de nombres, le théorème est établi dans [2] (modulo la remarque ci-dessus sur les définitions légèrement différentes des minima, différence qui joue en notre faveur). Pour un corps de fonctions, on consultera [38], corollaire 1. Ces deux références traitent seulement le cas d'un fibré vectoriel hermitien (c.-à-d. $\operatorname{vr}(\bar{E})=1$ ). Le cas général s'en déduit au moyen du fibré de John $J(\bar{E})$ en observant que $\lambda_{i}(\bar{E}) \leq \lambda_{i}(J(\bar{E}))$ et en utilisant la définition de $\operatorname{vr}(\bar{E})$.

Il est possible d'obtenir également une minoration du produit de ces minima en observant que, pour tout $\varepsilon>0$, il existe des éléments $a_{1}(\varepsilon), \ldots, a_{n}(\varepsilon) \in k_{\mathrm{A}}$ et une base $\left(e_{1}(\varepsilon), \ldots, e_{n}(\varepsilon)\right)$ de $E$ tels que, pour tout $i \in\{1, \ldots, n\}$, on a $\left|a_{i}(\varepsilon)\right|_{\mathbf{A}} \in\left[\lambda_{i}(\bar{E}), \lambda_{i}(\bar{E})+\varepsilon\right]$ et, pour toute place $v$ de $k$, $\left\|e_{i}(\varepsilon)\right\|_{v} \leq\left|a_{i}(\varepsilon)\right|_{v}$. De ce fait, le volume de la boule unité de $\bar{E}$ - volume relatif à la mesure de Haar $\mu_{E_{\mathrm{A}}}$ définie au paragraphe 2.1 - est minoré

$$
\operatorname{vol}(\mathbb{B}(\bar{E})) \geq \operatorname{vol}_{n}\left(b_{n, \mathbf{R}}^{1}\right)^{r_{1}}\left(2^{n} \operatorname{vol}_{2 n}\left(b_{n, \mathbf{C}}^{1}\right)\right)^{r_{2}}\left(\prod_{v, i}\left\|e_{i}(\varepsilon)\right\|_{v}^{n_{v}}\right)^{-1}
$$

car, pour tout $x=\sum_{i=1}^{n} x_{i} e_{i}(\varepsilon) \in E \otimes k_{v}$, on a

$$
\|x\|_{E, v} \leq\left\{\begin{array}{cl}
\max _{1 \leq i \leq n}\left\{\left|x_{i}\right|_{v}\left\|e_{i}(\varepsilon)\right\|_{v}\right\} & \text { si } v \text { est ultramétrique } \\
\sum_{i=1}^{n}\left|x_{i}\right|_{v}\left\|e_{i}(\varepsilon)\right\|_{v} & \text { si } v \text { est archimétrique }
\end{array}\right.
$$

et la formule (22) permet de calculer le degré de $E$ muni des métriques à droite. L'estimation (42) entraîne (avec $\varepsilon \rightarrow 0$ )

$$
\begin{aligned}
\lambda_{1}(\bar{E}) \cdots \lambda_{n}(\bar{E}) & \frac{\operatorname{vol}(\mathbb{B}(\bar{E}))}{\operatorname{covol}(E)} \\
& \geq \begin{cases}\frac{2^{n D} \pi^{n r_{2}}}{(n !)^{r_{1}}(2 n !)^{r_{2}}\left|D_{k}\right|^{n / 2}} & \text { si } k \text { est un corps de nombres, } \\
q^{-n(g(k)-1)} & \text { si } k \text { est un corps de fonctions. }\end{cases}
\end{aligned}
$$


Les quantités qui sont à droite proviennent des formules (4) et (5) ainsi que du choix de la mesure $\mu$ sur $k_{\mathrm{A}}$ définie au paragraphe 2.1. Dans le cas d'un corps de nombres, cette minoration est la même que celle de $\mathrm{E}$. Bombieri \& J. Vaaler [2], théorème 6.

Comme nous l'avons dit, le lien entre ces minima et les pentes de $\bar{E}$ a été précisé par T. Borek [3]. L'énoncé suivant est une généralisation aux fibrés vectoriels adéliques.

THÉORÈme 5.20. Soit $\bar{E}$ un fibré vectoriel adélique sur $\operatorname{Spec} k$, de dimension n. Posons

$$
C(n, k):=\frac{1}{D} \log \frac{\kappa^{n} \operatorname{covol}\left(k^{n}\right)}{\operatorname{vol} \mathbb{B}\left(k^{n},|\cdot|_{2}\right)}
$$

où $\kappa$ est la constante qui intervient dans le théorème 5.19. Alors, pour tout $i \in\{1, \ldots, n\}$, on $a$

$$
-\frac{i}{D} \log \Delta(\bar{E}) \leq \widehat{\mu}_{i}(\bar{E})+\frac{1}{D} \log \lambda_{i}(\bar{E}) \leq \frac{i}{n} C(n, k)+\frac{i}{D} \log \Delta(\bar{E}) .
$$

Nous n'allons pas refaire la preuve de cet énoncé qui - dans le cas hermitien - est l'objet du travail [3] de T. Borek. Même si ce dernier ne considère que des fibrés vectoriels hermitiens sur Spec $\mathcal{O}_{k}$, les méthodes de cet article se généralisent au cadre adélique car, comme nous l'avons vu, les degrés et les pentes définis ici donnent les mêmes invariants que ceux définis habituellement pour $\bar{E} \rightarrow \operatorname{Spec} \mathcal{O}_{k}$. De plus, l'on dispose également de la filtration canonique associée à un fibré adélique hermitien, qui est l'outil essentiel dans [3]. Le cas général d'un fibré vectoriel adélique quelconque se traite à partir du cas hermitien au moyen du fibré hermitien $\bar{E}_{\varepsilon}$ et des encadrements (36) et (41). On peut mentionner la formule asymptotique $C(n, k)=\frac{n}{2} \log n+\mathrm{O}(n)$ lorsque $n \rightarrow+\infty$ (le corps $k$ étant fixé), formule qui s'obtient au moyen de la mesure $\mu$ définie au $\S 2.1$ et des formules (4) et (5).

\section{Inégalités de pentes.}

L'objectif de ce paragraphe est de comparer les degrés et les pentes de fibrés vectoriels adéliques reliés entre eux par une application linéaire. En réalité nous avons déjà comparé de telles données lorsque l'application sous-jacente est une inclusion. 
DÉFInition 6.1. Soit $\bar{E}, \bar{F}$ des fibrés vectoriels adéliques sur Spec $k$ et $\varphi: E \rightarrow F$ une application $k$-linéaire. La hauteur de $\varphi$ relative à $\bar{E}$ et $\bar{F}$, notée $h(\bar{E}, \bar{F} ; \varphi)$ ou, plus simplement, $h(\varphi)$ s'il n'y a pas d'ambiguité, est la somme

$$
h(\bar{E}, \bar{F} ; \varphi):=\frac{1}{D} \sum_{v} n_{v} \log \|\varphi\|_{v} .
$$

La norme $\|\varphi\|_{v}$ est la norme d'opérateur de l'application induite $\varphi_{v}: E \otimes_{k} k_{v} \rightarrow F \otimes_{k} k_{v}$ et la somme ci-dessus ne comporte qu'un nombre fini de termes non nuls.

La formule du produit assure l'invariance par multiplication par un scalaire non nul :

$$
\forall \lambda \in k \backslash\{0\}, \quad h(\bar{E}, \bar{F} ; \lambda \varphi)=h(\bar{E}, \bar{F} ; \varphi) .
$$

La hauteur est invariante par extension finie $K$ du corps de base $k$ :

$$
h\left(\overline{E_{K}}, \overline{F_{K}} ; \varphi_{K}\right)=h(\bar{E}, \bar{F} ; \varphi)
$$

car la norme d'opérateur de $\varphi$ en une place $w$ de $K$ est égale à celle en la place $v$ de $k$ correspondante et car $\sum_{w \mid v}\left[K_{w}: k_{v}\right]=[K: k]$. De plus, il est utile de noter les deux encadrements suivants :

$$
-\log (1+\varepsilon)-\frac{1}{D} \log \Delta(\bar{E})+h(\bar{E}, \bar{F} ; \varphi) \leq h\left(\bar{E}, \bar{F}_{\varepsilon} ; \varphi\right) \leq h(\bar{E}, \bar{F} ; \varphi)
$$

et

$$
h(\bar{E}, \bar{F} ; \varphi) \leq h\left(\bar{E}_{\varepsilon}, \bar{F} ; \varphi\right) \leq h(\bar{E}, \bar{F} ; \varphi)+\frac{1}{D} \log \Delta(\bar{E})+\log (1+\varepsilon) .
$$

Lemme 6.2. Si $\varphi: E \rightarrow F$ est un isomorphisme entre deux droites vectorielles alors

$$
\widehat{\operatorname{deg}}_{\mathrm{n}} \bar{E}=\widehat{\operatorname{deg}}_{\mathrm{n}} \bar{F}+h(\bar{E}, \bar{F} ; \varphi) .
$$

La raison en est que, pour tout $x \in E_{v} \backslash\{0\}$, on a $\|\varphi\|_{v}=\|\varphi(x)\|_{F, v} /\|x\|_{E, v}$. On conclut au moyen du lemme 4.4 par exemple. En particulier, si $\bar{E}$ et $\bar{F}$ sont des fibrés adéliques hermitiens (de dimension quelconque), et si $\varphi: E \rightarrow F$ est un isomorphisme, le lemme 4.5 entraine

$$
\widehat{\operatorname{deg}}_{\mathrm{n}} \bar{E}=\widehat{\operatorname{deg}}_{\mathrm{n}} \bar{F}+h(\operatorname{det} \bar{E}, \operatorname{det} \bar{F} ; \operatorname{det} \varphi) .
$$


LEMME 6.3. Si $\varphi: E \rightarrow F$ est un isomorphisme alors les pentes normalisées de $\bar{E}$ et $\bar{F}$ vérifient

$$
\widehat{\mu}_{\mathrm{n}}(\bar{E}) \leq \widehat{\mu}_{\mathrm{n}}(\bar{F})+h(\bar{E}, \bar{F} ; \varphi) .
$$

DÉmonstration. On peut supposer $E=F=k^{n}$ et $\varphi$ s'identifie à une matrice de $\mathrm{GL}_{n}(k)$. En toute place $v$ de $k$, on a l'inclusion

$$
\left\{x \in k_{v}^{n} ;\|x\|_{E, v} \leq \frac{1}{\|\varphi\|_{v}}\right\} \subseteq\left\{x \in k_{v}^{n} ;\|\varphi(x)\|_{F, v} \leq 1\right\}
$$

Ce qui, pour une mesure de Haar $\operatorname{vol}_{v}$ quelconque sur $k_{v}^{n}$, entraîne

$$
\left(\frac{1}{\|\varphi\|_{v}}\right)^{n_{v} n} \frac{\operatorname{vol}_{v}\left(\mathbf{B}\left(k_{v}^{n},\|\cdot\|_{E, v}\right)\right)}{\operatorname{vol}_{v}\left(\mathbf{B}\left(k_{v}^{n},|\cdot|_{2, v}\right)\right)} \leq \frac{\operatorname{vol}_{v}\left(\mathbf{B}\left(k_{v}^{n},\|\cdot\|_{F, v}\right)\right)}{\operatorname{vol}_{v}\left(\mathbf{B}\left(k_{v}^{n},|\cdot|_{2, v}\right)\right)}|\operatorname{det} \varphi|_{v}^{-n_{v}} .
$$

L'inégalité souhaitée s'en déduit au moyen de la formule du produit appliquée à det $\varphi \in k \backslash\{0\}$.

LEMme 6.4. Soit $\bar{E}, \bar{F}$ des fibrés vectoriels adéliques et $\varphi: E \rightarrow F$ une application linéaire. Si $\varphi$ est injective alors

$$
\widehat{\mu}_{\max }(\bar{E}) \leq \widehat{\mu}_{\max }(\bar{F})+h(\varphi) .
$$

REMARQUE 6.5. La convention $\widehat{\mu}(0)=-\infty$ trouve une justification ici car l'on peut choisir $E=\{0\}$ dans cet énoncé.

DÉmonstration. Soit $\overline{E^{\prime}} \subseteq \bar{E}$. On pose $F^{\prime}=\varphi\left(E^{\prime}\right)$, espace vectoriel sur $k$ que l'on munit des métriques induites par $\bar{F}$. Ainsi, grâce à l'hypothèse d'injectivité, $\varphi$ induit un isomorphisme $\varphi_{\mid E^{\prime}}^{\mid F^{\prime}}$ entre $E^{\prime}$ et $F^{\prime}$. La majoration du lemme précédent donne alors

$$
\begin{aligned}
\widehat{\mu}_{\mathrm{n}}\left(\overline{E^{\prime}}\right) & \leq \widehat{\mu}_{\mathrm{n}}\left(\overline{F^{\prime}}\right)+h\left(\overline{E^{\prime}}, \overline{F^{\prime}} ; \varphi_{\mid E^{\prime}}^{\mid F^{\prime}}\right) \\
& \leq \widehat{\mu}_{\max }(\bar{F})+h(\bar{E}, \bar{F} ; \varphi) .
\end{aligned}
$$

On choisit alors convenablement $E^{\prime}$ pour obtenir la pente maximale de $\bar{E}$ à gauche.

Ily a plusieurs façons d'étendre cette inégalité. La première - qui est cœur de ce quel'on appelle méthode des pentes - consiste à considérer une filtration

$$
0=F_{N} \subseteq F_{N-1} \subseteq \cdots \subseteq F_{0}:=F
$$


d'un espace $F$ par des $k$-espaces vectoriels $F_{i}$. On ne suppose pas que $F$ est muni d'une structure de fibré vectoriel adélique. En revanche, chacun des espaces quotients $G_{i}:=F_{i-1} / F_{i}, 1 \leq i \leq N$, est muni de normes $\|\cdot\|_{i, v}$ aux places de $k$ telles que $\left(G_{i},\left(\|\cdot\|_{i, v}\right)_{v}\right)$ soit un fibré vectoriel adélique. Soit $\bar{E}$ un fibré vectoriel adélique sur Spec $k$ de dimension $n$ et $\varphi: E \rightarrow F$ une application $k$-linéaire. On pose

$$
\forall i \in\{1, \ldots, N+1\}, \quad E_{i}:=\varphi^{-1}\left(F_{i-1}\right) .
$$

Chacun des espaces $E_{i}$ est pourvu de la structure adélique $\overline{E_{i}}$ induite par $\bar{E}$. On pose $E_{N+1}:=\{0\}$. Soit $\varphi_{i}: E_{i} \rightarrow G_{i}$ la composée de la restriction de $\varphi$ à $E_{i}$ et de la projection canonique $F_{i-1} \rightarrow F_{i-1} / F_{i}$.

Proposition 6.6. Avec les notations ci-dessus, si $\varphi$ est injective alors

$$
\widehat{\mu}_{\mathrm{n}}(\bar{E}) \leq \sum_{i=1}^{N} \frac{\operatorname{dim}\left(E_{i} / E_{i+1}\right)}{n}\left\{\widehat{\mu}_{\max }\left(\overline{G_{i}}\right)+h\left(\overline{E_{i}}, \overline{G_{i}} ; \varphi_{i}\right)\right\}+\frac{1}{D} \log \operatorname{vr}(\bar{E}) .
$$

Cette majoration n'est pas tout à fait une généralisation du lemme 6.4 à cause du terme $\frac{1}{D} \log \operatorname{vr}(\bar{E})$ qui subsiste même lorsque $N=1$.

DÉmonstration. Chacune des applications $\varphi_{i}$ se factorise en une injection $\widetilde{\varphi}_{i}: E_{i} / E_{i+1} \hookrightarrow G_{i}$ à laquelle l'on peut appliquer le lemme 6.4 . On a donc en particulier

$$
\begin{aligned}
\widehat{\operatorname{deg}}_{\mathrm{n}}\left(\overline{E_{i} / E_{i+1}}\right) & \leq \operatorname{dim}\left(E_{i} / E_{i+1}\right)\left\{\widehat{\mu}_{\max }\left(\overline{G_{i}}\right)+h\left(\overline{E_{i} / E_{i+1}}, \overline{G_{i}} ; \widetilde{\varphi}_{i}\right)\right\} \\
& \leq \operatorname{dim}\left(E_{i} / E_{i+1}\right)\left\{\widehat{\mu}_{\max }\left(\overline{G_{i}}\right)+h\left(\overline{E_{i}}, \overline{G_{i}} ; \varphi_{i}\right)\right\}
\end{aligned}
$$

La difficulté est que le degré du quotient à gauche n'est pas en général la différence des degrés sauf si $\overline{E_{i}}$ est hermitien (voir proposition 4.22). Autrement dit, si $\bar{E}$ est hermitien, la proposition est établie en sommant les inégalités ci-dessus de $i=1$ à $N$. Le cas général s'en déduit alors en appliquant l'inégalité (49) à $J(\bar{E})$ et en utilisant la proposition 4.9 ainsi que la majoration $h\left(\left(E_{i},|\cdot|_{J(\bar{E})}\right), \overline{G_{i}} ; \varphi_{i}\right) \leq h\left(\overline{E_{i}}, \overline{G_{i}} ; \varphi_{i}\right)$.

Une autre façon de généraliser le lemme 6.4 est de considérer des pentes autres que la pente maximale, et même de supprimer l'hypothèse d'injectivité de $\varphi$, au prix de quelques modifications données dans l'énoncé suivant. 
Proposition 6.7. Soit $\bar{E}, \bar{F}$ des fibrés vectoriels adéliques et $\varphi: E \rightarrow F$ une application $k$-linéaire. Alors, pour tout entier $i \geq 1$ inférieur au rang de $\varphi$, la pente $\widehat{\mu}_{i+\operatorname{dim} \operatorname{ker} \varphi}(\bar{E})$ est inférieure $\grave{a}$

$$
\widehat{\mu}_{i}(\bar{F})+\frac{i}{D} \log \Delta(\bar{F})+\frac{(i+\operatorname{dim} \operatorname{ker} \varphi)}{D} \log \Delta(\bar{E})+h(\bar{E}, \bar{F} ; \varphi) .
$$

DÉMONSTRATION. On commence par montrer que

$$
\widehat{\mu}_{i+\operatorname{dim} \operatorname{ker} \varphi}(\bar{E}) \leq \widehat{\mu}_{i}(\bar{F})+h(\bar{E}, \bar{F} ; \varphi)
$$

lorsque $\bar{E}$ et $\bar{F}$ sont hermitiens. Tout d'abord, montrons que l'on peut supposer que $\varphi$ est injective. En effet, le quotient $E / \operatorname{ker} \varphi$ s'injecte dans $F$. Grâce à la formule (38) du minimax (qui est une égalité dans le cas hermitien), pour tout $\varepsilon>0$, il existe un sous-fibré adélique hermitien $\bar{E}_{\varepsilon}$ de $\bar{E}$, de dimension inférieure à $\operatorname{dim} \operatorname{ker} \varphi+i-1$ et contenant $\operatorname{ker} \varphi$, tel que

$$
\widehat{\mu}_{\max } \overline{\left(\frac{E / \operatorname{ker} \varphi}{E_{\varepsilon} / \operatorname{ker} \varphi}\right)} \leq \widehat{\mu}_{i}(\overline{E / \operatorname{ker} \varphi})+\varepsilon .
$$

Or l'application $p: E / E_{\varepsilon} \rightarrow(E / \operatorname{ker} \varphi) /\left(E_{\varepsilon} / \operatorname{ker} \varphi\right)$ est un isomorphisme de hauteur négative car la norme d'opérateur de $p$ est plus petite que 1 en chaque place de $k$. La pente maximale de

$$
\overline{\left(\frac{E / \operatorname{ker} \varphi}{E_{\varepsilon} / \operatorname{ker} \varphi}\right)}
$$

est donc supérieure à celle de $\overline{E / E_{\varepsilon}}$ par le lemme 6.4 , elle-même plus grande que $\widehat{\mu}_{i+\operatorname{dim} \operatorname{ker} \varphi}(\bar{E})$ toujours en vertu de la formule (38). On peut alors faire tendre $\varepsilon$ vers 0 et constater que

$$
\widehat{\mu}_{i+\operatorname{dim} \operatorname{ker} \varphi}(\bar{E}) \leq \widehat{\mu}_{i}(\overline{E / \operatorname{ker} \varphi}) .
$$

Comme $h(\overline{E / \operatorname{ker} \varphi}, \bar{F} ; \varphi) \leq h(\bar{E}, \bar{F} ; \varphi)$, il suffit donc bien de traiter le cas $\operatorname{ker} \varphi=\{0\}$. Dans ce cas, on utilise à nouveau la formule (38) en observant que si $E_{2} \subseteq E_{1} \subseteq E$ avec $\operatorname{dim} E_{1} \geq i>\operatorname{dim} E_{2}$ alors $\varphi\left(E_{2}\right) \subseteq \varphi\left(E_{1}\right) \subseteq F$ avec $\operatorname{dim} \varphi\left(E_{1}\right) \geq \imath>\operatorname{dim} \varphi\left(E_{2}\right)$. La pente maximale de $\overline{E / E_{2}}$ est à la fois plus petite que $\widehat{\mu}_{\max }\left(\overline{F / \varphi\left(E_{2}\right)}\right)+h(\bar{E}, \bar{F} ; \varphi)$ (lemme 6.4 ) et plus grande que $\widehat{\mu}_{i}(\bar{E})$ (formule (38)). D'où l'on déduit $\widehat{\mu}_{i}(\bar{E}) \leq \widehat{\mu}_{i}(\bar{F})+h(\bar{E}, \bar{F} ; \varphi)$ en faisant varier $E_{2}$, l'espace $\varphi\left(E_{2}\right)$ parcourant les sous-espaces vectoriels de $\varphi(E)$ de dimension inférieure à $i-1$.

Une fois la majoration établie dans le cas hermitien, on l'applique aux fibrés adéliques hermitiens $\bar{E}_{\varepsilon}$ et $\bar{F}_{\varepsilon}$ (introduits après les relations (28)), 
puis l'on utilise l'encadrement (36) ainsi que les évaluations (44) et (45) de la hauteur de $\varphi$ pour en déduire le cas général.

Corollaire 6.8. Soit $\bar{E}, \bar{F}$ des fibrés vectoriels adéliques et $\varphi: E \rightarrow F$ une application $k$-linéaire. On note $m$ la dimension de $F$. Si $\varphi$ est surjective alors la pente maximale $\widehat{\mu}_{\max }(\bar{F})$ est inférieure à

$$
\widehat{\operatorname{deg}}_{\mathrm{n}} \bar{F}-(m-1) \widehat{\mu}_{\text {min }}(\bar{E})+(m-1) h(\bar{E}, \bar{F} ; \varphi)+\frac{m}{D} \log (\Delta(\bar{E}) \Delta(\bar{F})) .
$$

DÉmonstration. Des formules $\widehat{\operatorname{deg}}_{\mathrm{n}} \bar{F}=\sum_{i=1}^{m} \widehat{\mu}_{i}(\bar{F})$ et $\widehat{\mu}_{1}(\bar{F})=\widehat{\mu}_{\max }(\bar{F})$ (lemme 5.10) l'on déduit la majoration

$$
\widehat{\mu}_{\max }(\bar{F}) \leq \widehat{\operatorname{deg}}_{\mathrm{n}} \bar{F}-(m-1) \widehat{\mu}_{m}(\bar{F}) .
$$

La proposition 6.7 appliquée avec $i=m=\operatorname{rg} \varphi$ fournit une minoration de $\widehat{\mu}_{m}(\bar{F})$ qui est trop faible pour prouver le corollaire à cause des termes d'erreurs qui se sont ajoutés. On va donc commencer par utiliser l'estimation (50) avec le fibré hermitien $\bar{F}_{\varepsilon}$ construit à la suite de (28) au lieu de $\bar{F}$. On a vu que $\widehat{\mu}_{\max }(\bar{F}) \leq \widehat{\mu}_{\max }\left(\bar{F}_{\varepsilon}\right)$ (voir ce qui suit la définition 5.4) et la proposition 6.7 appliquée au morphisme $\varphi: \bar{E}_{\varepsilon} \rightarrow \bar{F}_{\varepsilon}$ et $i=m=\operatorname{rg} \varphi$ fournit la minoration

$$
\widehat{\mu}_{m}\left(\bar{F}_{\varepsilon}\right) \geq \widehat{\mu}_{n}\left(\bar{E}_{\varepsilon}\right)-h\left(\bar{E}_{\varepsilon}, \bar{F}_{\varepsilon} ; \varphi\right) .
$$

On obtient ainsi

$$
\begin{aligned}
\widehat{\mu}_{\max }(\bar{F}) \leq \widehat{\mu}_{\max }\left(\bar{F}_{\varepsilon}\right) & \leq \widehat{\operatorname{deg}}_{\mathrm{n}} \bar{F}_{\varepsilon}-(m-1) \widehat{\mu}_{m}\left(\bar{F}_{\varepsilon}\right) \\
& \leq \widehat{\operatorname{deg}}_{\mathrm{n}} \bar{F}_{\varepsilon}-(m-1) \widehat{\mu}_{n}\left(\bar{E}_{\varepsilon}\right)+(m-1) h\left(\bar{E}_{\varepsilon}, \bar{F}_{\varepsilon} ; \varphi\right) .
\end{aligned}
$$

D'après l'observation (28), on a $\widehat{\operatorname{deg}}_{\mathrm{n}} \bar{F}_{\varepsilon} \leq \widehat{\operatorname{deg}}_{\mathrm{n}} \bar{F}+\frac{m}{D} \log \Delta(\bar{F})+m \log (1+\varepsilon)$. De plus, les estimations (44) et (45) pour la hauteur donnent

$$
h\left(\bar{E}_{\varepsilon}, \bar{F}_{\varepsilon} ; \varphi\right) \leq h\left(\bar{E}_{\varepsilon}, \bar{F} ; \varphi\right) \leq h(\bar{E}, \bar{F} ; \varphi)+\frac{1}{D} \log \Delta(\bar{E})+\log (1+\varepsilon) .
$$

Enfin, grâce au lemme 5.11 pour le fibré hermitien $\bar{E}_{\varepsilon}$, on a

$$
\widehat{\mu}_{n}\left(\bar{E}_{\varepsilon}\right)=\min _{E^{\prime} \subsetneq E} \widehat{\mu}_{\mathrm{n}}\left(E / E^{\prime},|\cdot|_{\varepsilon}\right) \geq \min _{E^{\prime} \subsetneq E} \widehat{\mu}_{\mathrm{n}}\left(E / E^{\prime},\|\cdot\|_{\bar{E}}\right)=\widehat{\mu}_{\min }(\bar{E}) .
$$

La majoration souhaitée pour la pente maximale $\widehat{\mu}_{\max }(\bar{F})$ découle de ces estimations que l'on reporte dans la majoration (51), en faisant tendre ensuite $\varepsilon$ vers 0 . 


\section{Pentes maximales des puissances symétriques.}

L'objectif de ce paragraphe est de montrer l'énoncé suivant.

THÉoRÈme 7.1. Soit $\bar{E}$ un fibré adélique hermitien de dimension $n \geq 1$. Pour tout entier $\ell \geq 0$, la pente maximale de la $\ell^{\text {ème }}$ puissance symétrique $S^{\ell}(\bar{E})$ vérifie

1) si $k$ est un corps de fonctions alors on a $\widehat{\mu}_{\max }\left(S^{\ell}(\bar{E})\right)=\ell \widehat{\mu}_{\max }(\bar{E})$.

2) si $k$ est un corps de nombres alors

$$
0 \leq \widehat{\mu}_{\max }\left(S^{\ell}(\bar{E})\right)-\ell \widehat{\mu}_{\max }(\bar{E}) \leq 2 \ell n \log n .
$$

Avant d'effectuer la démonstration de ce théorème, rassemblons deux énoncés préparatoires, d'intérêt indépendant.

Pour $\mathbf{i}=\left(i_{1}, \ldots, i_{n}\right) \in \mathbf{N}^{n}$, on note $|\mathbf{i}|$ la longueur de $\mathbf{i}$ définie par $|\mathbf{i}|:=i_{1}+\cdots+i_{n}$ et $\mathbf{i} !:=i_{1} ! \cdots i_{n}$ !. Étant donné $\ell \in \mathbf{N}$, si $A$ est la matrice d'un endomorphisme $u$ dans une base $\left(e_{1}, \ldots, e_{n}\right)$ de $k^{n}$, la matrice $S^{\ell}(A)$ est celle qui représente $S^{\ell}(u)$ dans la base $e_{1}^{i_{1}} \ldots e_{n}^{i_{n}}$, où les $n$-uplets $\left(i_{1}, \ldots, i_{n}\right) \in \mathbf{N}^{n}$ sont de longueur $\ell$ et ordonnés lexicographiquement.

Lemme 7.2. Soit $v$ une place de $k$ et $u_{v}$ un endomorphisme de $k_{v}^{n}$. On suppose $k_{v}^{n}$ muni de la norme $|\cdot|_{2, v}$ et on note $\|.\|_{v}$ la norme d'opérateur des endomorphismes de $\left(k_{v}^{n},|\cdot|_{2, v}\right)$ si v est ultramétrique ou la norme de Hilbert-Schmidt si $v$ est archimédienne.

(i) Si $u_{v}$ est un isomorphisme alors

$$
\left\|u_{v}^{-1}\right\|_{v} \leq \frac{\left\|u_{v}\right\|_{v}^{n-1}}{\left|\operatorname{det} u_{v}\right|_{v}} .
$$

(ii) Pour tout entier naturel $\ell$, on a

$$
\left.\operatorname{det} S^{\ell}\left(u_{v}\right)=\left(\operatorname{det} u_{v}\right)^{(\ell+n-1}{ }_{n}\right) .
$$

Éléments de démonstration.

(i) En une place finie $v$, si l'on note $A_{v}$ la matrice qui représente $u_{v}$ dans la base canonique de $k_{v}^{n}$, l'estimation (52) est la conséquence de la formule matricielle $A_{v}^{-1}=\frac{{ }^{\mathrm{t}} \operatorname{com} A_{v}}{\operatorname{det} A_{v}}$ où ${ }^{\mathrm{t}} \operatorname{com} A_{v}$ désigne la transposée de la comatrice de $A_{v}$. Si $v$ est archimédienne, on observe que la norme de Hilbert-Schmidt $\left\|u_{v}\right\|_{v}$ est la racine carrée de la somme des valeurs propres de l'opérateur hermitien ${ }^{\mathrm{t}} \overline{u_{v}} u_{v}$ qui est défini positif. En remarquant que ${ }^{\mathrm{t}} \overline{u_{v}} u_{v}$ et $u_{v}{ }^{\mathrm{t}} \overline{u_{v}}$ ont 
le même spectre $\lambda_{1} \leq \cdots \leq \lambda_{n}$, l'inégalité (52) n'est rien d'autre que

$$
\left(\sum_{i=1}^{n} \lambda_{i}^{-1}\right)\left(\lambda_{1} \cdots \lambda_{n}\right) \leq\left(\sum_{i=1}^{n} \lambda_{i}\right)^{n-1}
$$

(ii) Pour démontrer cette formule, on peut par exemple choisir une base trigonalisante de $u_{v}$ dans une extension algébrique de $k_{v}$ et observer que le monôme symétrique $\prod_{|\mathbf{i}|=\ell} X_{1}^{i_{1}} \cdots X_{n}^{i_{n}}$ égale $\left.\left(X_{1} \cdots X_{n}\right)^{(\ell+n-1}{ }_{n}\right)$.

Le second lemme requiert deux notations supplémentaires. On pose

$$
\delta:= \begin{cases}0 & \text { si } k \text { est un corps de fonctions } \\ 1 & \text { si } k \text { est un corps de nombres }\end{cases}
$$

et, pour $n, \ell \in \mathbf{N}$, on définit

$$
\left.\gamma_{n, \ell}:=\left(\prod_{|\mathbf{i}|=\ell} \frac{\ell !}{\mathbf{i} !}\right)^{\left(\begin{array}{c}
\ell+n-1 \\
n-1
\end{array}\right)^{-1}} \quad \text { (dans ce produit, } \mathbf{i} \in \mathbf{N}^{n}\right) .
$$

On connait une estimation asymptotique du logarithme de $\gamma_{n, \ell}$ lorsque $\ell \rightarrow+\infty$ et $n$ fixé, que l'on peut écrire en fonction des nombres harmoniques $H_{n}:=\sum_{i=1}^{n} \frac{1}{i}$. La quantité $\log \gamma_{n, \ell}$ est alors égale à $\left(H_{n}-1\right)(\ell+o(\ell))$ lorsque $\ell \rightarrow+\infty$ (voir annexe).

Lemme 7.3. Soit $\ell \in \mathbf{N} \backslash\{0\}$ et $\bar{E}$ un fibré vectoriel adélique de dimension $n \geq 1$. Alors, pour toute norme tensorielle adélique hermitienne $\alpha$ d'ordre $\ell$, on a

$$
\left|\widehat{\mu}_{\mathrm{n}}\left(S_{\alpha}^{\ell}(\bar{E})\right)-\ell \widehat{\mu}_{\mathrm{n}}(\bar{E})-\frac{\delta}{2} \log \gamma_{n, \ell}\right| \leq \frac{\ell}{D} \log \Delta(\bar{E}) .
$$

DÉmonstration. Supposons dans un premier temps que $\bar{E}$ est hermitien. Dans ce cas, on peut omettre la référence à $\alpha$ dans la notation $S_{\alpha}^{\ell}(\bar{E})$ (qui n'en dépend pas, car $\alpha$ est hermitienne). Il s'agit de montrer que

$$
\widehat{\mu}_{\mathrm{n}}\left(S^{\ell}(\bar{E})\right)=\ell \widehat{\mu}_{\mathrm{n}}(\bar{E})+\frac{\delta}{2} \log \gamma_{n, \ell} .
$$

Quitte à fixer une $k$-base de $E$, on peut identifier $E$ à $k^{n}$. Il existe une matrice adélique $A=\left(A_{v}\right)_{v} \in \mathrm{GL}_{n}\left(k_{\mathrm{A}}\right)$ telle que, pour toute place $v$ de $k$ et tout $x \in k_{v}^{n}$, on ait $\|x\|_{E, v}=\left|A_{v} \cdot x\right|_{2, v}$. Les métriques sur $S^{\ell}(\bar{E})$ sont déterminées par $S^{\ell}(A)$, au sens où $S^{\ell}(\bar{E})$ est l'image par $S^{\ell}(A)$ du fibré adélique hermitien 
$S_{\alpha}^{\ell}\left(k^{n},|\cdot|_{2}\right)$. L'égalité (46) et le lemme 4.4 entraînent alors

$$
\widehat{\operatorname{deg}}_{\mathrm{n}} S^{\ell}\left(k^{n},|\cdot|_{2}\right)=\widehat{\operatorname{deg}}_{\mathrm{n}} S^{\ell}(\bar{E})+\frac{1}{D} \sum_{v} n_{v} \log \frac{1}{\left|\operatorname{det} S^{\ell}\left(A_{v}\right)\right|_{v}} .
$$

Le lemme 7.2 (ii) permet de calculer le déterminant de $S^{\ell}\left(A_{v}\right)$. En observant que $\operatorname{dim} S^{\ell}(E)=\left(\begin{array}{c}\ell+n-1 \\ n-1\end{array}\right)$ et en appliquant à nouveau l'égalité (46) à $\bar{E}$ et $\left(k^{n},|\cdot|_{2}\right)$, on obtient

$$
\widehat{\mu}_{\mathrm{n}}\left(S^{\ell}(\bar{E})\right)=\ell \widehat{\mu}_{\mathrm{n}}(\bar{E})+\widehat{\mu}_{\mathrm{n}}\left(S^{\ell}\left(k^{n},|\cdot|_{2}\right)\right) .
$$

Ce dernier terme donne la contribution $\frac{1}{2} \log \gamma_{n, \ell}$ aux (éventuelles) places archimédiennes de $k$. Ceci est une conséquence du calcul local suivant. Soit $v$ une place de $k$. Si $\left(e_{1}, \ldots, e_{n}\right)$ désigne la base canonique de $k^{n}$ alors, pour tout $\mathbf{i}=\left(i_{1}, \ldots, i_{n}\right) \in \mathbf{N}^{n}$, la norme de l'élément $e_{1}^{i_{1}} \cdots e_{n}^{i_{n}} \in S^{\ell}\left(k^{n},|\cdot|_{2}\right)$ vaut $(i ! / \ell !)^{1 / 2}$ si $v$ est archimédienne et 1 sinon (voir $\S 3.3$ ). La formule souhaitée étant établie dans le cas hermitien, le passage au cas général s'effectue grâce aux fibrés de John et Löwner associés à $\bar{E}$, en observant que $S^{\ell}(L(\bar{E})) \preceq S_{\alpha}^{\ell}(\bar{E}) \preceq S^{\ell}(J(\bar{E}))$. On obtient alors

$$
-\frac{\ell}{D} \log \operatorname{vr}(\bar{E}) \leq \widehat{\mu}_{\mathrm{n}}\left(S_{\alpha}^{\ell}(\bar{E})\right)-\ell \widehat{\mu}_{\mathrm{n}}(\bar{E})-\frac{\delta}{2} \log \gamma_{n, \ell} \leq \frac{\ell}{D} \log \widetilde{\operatorname{vr}}(\bar{E})
$$

(voir (26) et la proposition 4.9). On conclut au moyen de la majoration $\max \{\operatorname{vr}(\bar{E}), \widetilde{\operatorname{vr}}(\bar{E})\} \leq \Delta(\bar{E})$ (voir $(27))$.

\section{Démonstration du théorème 7.1.}

La première inégalité $\ell \widehat{\mu}_{\max }(\bar{E}) \leq \widehat{\mu}_{\max }\left(S^{\ell}(\bar{E})\right)$ est une conséquence directe du lemme 7.3 (cas hermitien) :

$$
\forall \bar{F} \subseteq \bar{E}, \quad \ell \widehat{\mu}_{\mathrm{n}}(\bar{F}) \leq \widehat{\mu}_{\mathrm{n}}\left(S^{\ell}(\bar{F})\right) \leq \widehat{\mu}_{\max }\left(S^{\ell}(\bar{E})\right) .
$$

Le résultat se déduit de la définition de la pente maximale de $\bar{E}$. Montrons maintenant que

$$
\widehat{\mu}_{\max }\left(S^{\ell}(\bar{E})\right)-\ell \widehat{\mu}_{\max }(\bar{E}) \leq 2 \ell \delta n \log n
$$

( $\delta$ est défini par (53)). D'après le lemme de Siegel absolu (4.14), pour tout $\varepsilon>0$, il existe une $\bar{k}$-base $e_{1}, \ldots, e_{n}$ de $E \otimes \bar{k}$ telle que

$$
h_{\bar{E}}\left(e_{1}\right)+\cdots+h_{\bar{E}}\left(e_{n}\right)+\widehat{\operatorname{deg}}_{\mathrm{n}} \operatorname{det} \bar{E} \leq \frac{\delta n}{2} \log n+\varepsilon .
$$

On peut supposer que les vecteurs $e_{1}, \ldots, e_{n}$ sont définis sur une extension finie $K$ de $k$. Soit $\overline{E_{0}}$ le fibré adélique hermitien dont l'espace sous-jacent $E_{0}$ 
est $E_{K}$ et dont la norme d'un vecteur $x=\sum_{i} x_{i} e_{i} \in E \otimes_{k} \mathbf{C}_{v}$ en une place $w$ de $K$ au-dessus de $v$ est $\left(\sum_{i}\left|x_{i}\right|_{v}^{2}\left\|e_{i}\right\|_{E, v}^{2}\right)^{1 / 2}$ si $w$ est archimédienne et $\max _{i}\left\{\left|x_{i}\right|_{v}\left\|e_{i}\right\|_{E, v}\right\}$ sinon. Comme $\overline{E_{0}}$ et $\overline{E_{K}}$ sont des fibrés hermitiens avec le même espace sous-jacent, il existe une matrice $\Sigma=\left(\Sigma_{w}\right)_{w} \in \mathrm{GL}\left(E \otimes_{k} K_{\mathbf{A}}\right)$ telle que $\overline{E_{K}}=\Sigma \cdot \overline{E_{0}}$ (au sens donné peu après la définition du degré adélique, voir (22)). On note encore $\Sigma: E_{0} \rightarrow E_{K}$ l'application identique. Cet abus de notation se justifie par le fait que les normes d'opérateur ou de Hilbert-Schmidt de l'application $x \in E_{0} \otimes K_{w} \mapsto x \in E \otimes K_{w}$ sont celles de $\Sigma_{w}$ pour toute place $w$ de $K$. En appliquant le lemme 6.4 à l'application inverse de $S^{\ell}(\Sigma): S^{\ell}\left(E_{0}\right) \rightarrow S^{\ell}\left(E_{K}\right)$, on obtient :

$$
\begin{aligned}
\widehat{\mu}_{\max }\left(S^{\ell}(\bar{E})\right)=\widehat{\mu}_{\max }\left(S^{\ell}\left(\overline{E_{K}}\right)\right) & \leq \widehat{\mu}_{\max }\left(S^{\ell}\left(\overline{E_{0}}\right)\right)+h\left(S^{\ell}\left(\Sigma^{-1}\right)\right) \\
& \leq \widehat{\mu}_{\max }\left(S^{\ell}\left(\overline{E_{0}}\right)\right)+\ell h\left(\Sigma^{-1}\right) .
\end{aligned}
$$

Évaluons chacun des termes du membre de droite de cette inégalité. La pente maximale de $S^{\ell}\left(\overline{E_{0}}\right)$ se calcule en observant que $S^{\ell}\left(E_{0}\right)$ est la somme directe orthogonale des espaces $K \cdot e_{1}^{i_{1}} \cdots e_{n}^{i_{n}}$ pour $\mathbf{i}:=\left(i_{1}, \ldots, i_{n}\right)$ de longueur $\ell$. D’après la propriété $5.7,2)$, on a

$$
\begin{aligned}
\widehat{\mu}_{\max }\left(S^{\ell}\left(\overline{E_{0}}\right)\right) & =\max _{|\mathbf{i}|=\ell}\left\{\widehat{\operatorname{deg}}_{\mathrm{n}}\left(K \cdot e_{1}^{i_{1}} \cdots e_{n}^{i_{n}}\right)\right\} \\
& =\max _{|\mathbf{i}|=\ell}\left\{\sum_{j=1}^{n} i_{j} \widehat{\mu}_{\max }\left(\overline{K \cdot e_{j}}\right)+\frac{\delta}{2} \log \overline{\ell !} i_{1} ! \cdots i_{n} !\right\} .
\end{aligned}
$$

On en déduit

$$
\begin{aligned}
\widehat{\mu}_{\max }\left(S^{\ell}\left(\overline{E_{0}}\right)\right) & \leq \ell \max _{1 \leq i \leq n}\left\{\widehat{\mu}_{\max }\left(\overline{K \cdot e_{i}}\right)\right\}+\frac{\delta \ell}{2} \log n \\
& =\ell \widehat{\mu}_{\max }\left(\overline{E_{0}}\right)+\frac{\delta \ell}{2} \log n .
\end{aligned}
$$

Le lemme 6.4 appliqué à $\Sigma: E_{0} \rightarrow E_{K}$ donne $\widehat{\mu}_{\max }\left(\overline{E_{0}}\right) \leq \widehat{\mu}_{\max }(\bar{E})+h(\Sigma)$. La hauteur $h(\Sigma)$ est majorée par $\frac{\delta}{2} \log n$ car $\|\Sigma\|_{w} \leq \sqrt{n}$ si $w$ est archimédienne et $\|\Sigma\|_{w} \leq 1$ sinon. On obtient ainsi

$$
\widehat{\mu}_{\max }\left(S^{\ell}\left(\overline{E_{0}}\right)\right) \leq \ell\left(\widehat{\mu}_{\max }(\bar{E})+\delta \log n\right) .
$$

Par ailleurs, en ce qui concerne la hauteur de $\Sigma^{-1}$, on applique la majoration (52) du lemme 7.2. Compte tenu de l'estimation des normes de $\Sigma$, on en déduit la majoration

$$
h\left(\Sigma^{-1}\right) \leq \delta(n-1) \log \sqrt{n}-\frac{1}{D} \sum_{w \text { place de } K} n_{w} \log \left|\operatorname{det} \Sigma_{w}\right|_{w} .
$$


L'égalité (46) obtenue à la suite du lemme 6.2 montre que cette dernière somme est aussi la différence des degrés adéliques $\widehat{\operatorname{deg}}_{\mathrm{n}} \bar{E}-\widehat{\operatorname{deg}}_{\mathrm{n}} \overline{E_{0}}$, qui vaut $\widehat{\operatorname{deg}}_{\mathrm{n}} \operatorname{det} \bar{E}+h_{\bar{E}}\left(e_{1}\right)+\cdots+h_{\bar{E}}\left(e_{n}\right)$ car $\bar{E}$ est hermitien et le degré de $\overline{E_{0}}$ se calcule au moyen de la formule (22). Cette quantité est inférieure à $\frac{\delta n}{2} \log n+\varepsilon$ en vertu du choix de la base $\left(e_{1}, \ldots, e_{n}\right)$. La synthèse de ces informations conduit à l'estimation :

$$
\begin{aligned}
\widehat{\mu}_{\max }\left(S^{\ell}(\bar{E})\right) & \leq \ell\left(\widehat{\mu}_{\max }(\bar{E})+\delta \log n\right)+\ell\left(\delta(n-1) \log \sqrt{n}+\frac{\delta n}{2} \log n+\varepsilon\right) \\
& \leq \ell\left(\widehat{\mu}_{\max }(\bar{E})+\left(n+\frac{1}{2}\right) \delta \log n+\varepsilon\right) .
\end{aligned}
$$

Dans cette inégalité, on peut faire tendre $\varepsilon$ vers 0 et l'on obtient le résultat voulu.

Corollaire 7.4. Pour tout entier $\ell \geq 1$, pour toute norme tensorielle adélique hermitienne $\alpha$ d'ordre $\ell$ et tout fibré vectoriel adélique $\bar{E}$ sur Spec $k$, on $a$

$$
-\frac{\ell}{D} \log \Delta(\bar{E}) \leq \widehat{\mu}_{\max }\left(S_{\alpha}^{\ell}(\bar{E})\right)-\ell \widehat{\mu}_{\max }(\bar{E}) \leq \ell\left(2 n \delta \log n+\frac{1}{D} \log \Delta(\bar{E})\right) .
$$

DÉmonstration. En vertu du lemme 7.3, pour tout sous-fibré adélique $\bar{F} \subseteq \bar{E}$, on a

$$
\ell \widehat{\mu}_{\mathrm{n}}(\bar{F}) \leq \widehat{\mu}_{\mathrm{n}}\left(S_{\alpha}^{\ell}(\bar{F})\right)+\frac{\ell}{D} \log \Delta(\bar{F}) \quad \text { et } \quad \Delta(\bar{F}) \leq \Delta(\bar{E}) .
$$

A priori les métriques sur $S_{\alpha}^{\ell}(\bar{F})$ ne sont pas les restrictions des métriques de $S_{\alpha}^{\ell}(\bar{E})$ à $S^{\ell}(F)$. Toutefois, d'après la proposition 2.12 , on a $\left(F^{\otimes \ell},\|\cdot\|_{\bar{E}^{\otimes \alpha \ell}}\right) \preceq \bar{F}^{\otimes_{\alpha} \ell}$, d'où l'on déduit

$$
\left(S^{\ell}(F),\|\cdot\|_{S_{\alpha}^{\ell}(\bar{E})}\right) \preceq S_{\alpha}^{\ell}(\bar{F}) \quad \text { puis } \quad \widehat{\mu}_{\mathrm{n}}\left(S_{\alpha}^{\ell}(\bar{F})\right) \leq \widehat{\mu}_{\max }\left(S_{\alpha}^{\ell}(\bar{E})\right) .
$$

La première inégalité du corollaire 7.4 en découle. Pour la seconde estimation, on applique le théorème 7.1 au fibré adélique hermitien $\bar{E}_{\varepsilon}$ (voir (28)). Comme $\bar{E}_{\varepsilon} \preceq \bar{E}$, on en déduit $S^{\ell}\left(\bar{E}_{\varepsilon}\right) \preceq S_{\alpha}^{\ell}(\bar{E})$ (lemme 4.3) et donc

$$
\widehat{\mu}_{\max }\left(S_{\alpha}^{\ell}(\bar{E})\right) \leq \widehat{\mu}_{\max }\left(S^{\ell}\left(\bar{E}_{\varepsilon}\right)\right) .
$$

D'autre part, comme nous l'avons mentionné à la suite de la définition 5.4, la pente maximale de $\bar{E}_{\varepsilon}$ est majorée par celle de $\bar{E}$ plus $\log (1+\varepsilon)+\frac{1}{D} \log \Delta(\bar{E})$. Le résultat s'ensuit en faisant tendre $\varepsilon$ vers 0 . 
Remarque 7.5. Si $k$ est un corps de nombres, l'égalité $\widehat{\mu}_{\max }\left(S_{\alpha}^{\ell}(\bar{E})\right)=$ $=\ell \widehat{\mu}_{\max }(\bar{E})$ est en général fausse, même asymptotiquement lorsque $\ell \rightarrow+\infty$ ou même si $\bar{E}$ et $\alpha$ sont hermitiens. En effet l'estimation asymptotique $\log \gamma_{n, \ell} \underset{\ell \rightarrow+\infty}{\sim}\left(H_{n}-1\right) \ell$ et le lemme 7.3 entraînent la majoration

$$
\widehat{\mu}_{\mathrm{n}}(\bar{F})+\frac{1}{2}\left(H_{m}-1\right) \leq \frac{1}{D} \log \Delta(\bar{E})+\liminf _{\ell \rightarrow+\infty} \frac{\widehat{\mu}_{\max }\left(S^{\ell}(\bar{E})\right)}{\ell},
$$

vraie pour tout sous-fibré adélique $\bar{F} \subseteq \bar{E}$ de dimension $m \geq 1$. Aussi, lorsque la pente maximale de $\bar{E}$ est atteinte pour un fibré $\bar{F}$ de dimension $\geq 2$ (par exemple lorsque $\bar{E}$ est semi-stable) et si $\bar{E}$ est hermitien $(\Delta(\bar{E})=1$ ), on a

$$
\widehat{\mu}_{\max }(\bar{E})<\liminf _{\ell \rightarrow+\infty} \frac{\widehat{\mu}_{\max }\left(S^{\ell}(\bar{E})\right)}{\ell} .
$$

Ces propriétés des pentes et pentes maximales des puissances symétriques de $\bar{E}$ se transmettent aux fibrés des sections globales des puissances tensorielles du faisceau canonique $\mathcal{O}(1)$ de l'espace projectif $\mathbf{P}(E)$.

Plus précisément, soit $\bar{E}$ un fibré vectoriel adélique sur Spec $k$ de dimension $n \geq 1$. On note $\mathbf{P}(E)$ le schéma $\mathbf{S}(E)$ de morphisme structural $\pi: \mathbf{P}(E) \rightarrow$ Spec $k$ et $\mathcal{O}(1)$ le fibré en droites universel sur $\mathbf{P}(E)$. Pour toute place $v$ de $k$ et tout point $x \in \mathbf{P}(E)\left(\mathbf{C}_{v}\right)$, la surjection canonique $\pi^{*} E \rightarrow \mathcal{O}(1)$ confère une métrique $\|\cdot\|_{v . x}$ à la fibre $\mathcal{O}(1)_{x}$ par quotient. Et plus généralement, pour $\ell \in \mathbf{N} \backslash\{0\}$, on note $\|\cdot\|_{v, x, \ell}$ la norme induite sur $\mathcal{O}(\ell)_{x}=\mathcal{O}(1)_{x}^{\otimes \ell}$. En une place archimédienne $v$ de $k$, une mesure de Haar sur le groupe localement compact $\left(E \otimes_{k} \mathbf{C}_{v},+\right)$ induit une mesure sur l'ouvert $\left(E \otimes_{k} \mathbf{C}_{v}\right) \backslash\{0\}$, qui est un espace homogène sous l'action du groupe multiplicatif $\mathbf{C}_{v}^{*}$. On obtient de la sorte une unique mesure de probabilité $\mu_{E, v}$ sur $\mathbf{P}\left(E \otimes_{k} \mathbf{C}_{v}\right) \simeq\left(\left(E \otimes_{k} \mathbf{C}_{v}\right) \backslash\{0\}\right) / \mathbf{C}_{v}^{*}$. On peut munir alors le $k$-espace vectoriel $E_{\ell}$ des sections globales $\mathrm{H}^{0}(\mathbf{P}(E), \mathcal{O}(\ell))$ d'une structure de fibré adélique hermitien sur Spec $k$ en considérant les normes suivantes. Étant donné une place $v$ de $k$ et un élément $s$ de $E_{\ell} \otimes_{k} \mathbf{C}_{v}$, on pose

$$
\|s\|_{E_{\ell}, v}:= \begin{cases}\sup _{x \in \mathbf{P}(E)\left(\mathbf{C}_{v}\right)}\|s(x)\|_{v, x, \ell} & \text { si } v \text { est ultramétrique, } \\ \left(\int_{\mathbf{P}(E)\left(\mathbf{C}_{v}\right)}\|s(x)\|_{v, x, \ell}^{2} \mathrm{~d} \mu_{E, v}(x)\right)^{1 / 2} & \text { si } v \text { est archimédienne. }\end{cases}
$$

Aux places archimédiennes le choix peut sembler arbitraire mais il as- 
sure la compatibilité avec la littérature arakelovienne dans le cas où $\bar{E}$ est hermitien.

Lemme 7.6 (Lemme 4.3.6 de [4]). Soit $\bar{E}$ un fibré adélique hermitien sur Speck et $\ell$ un entier $\geq 1$. L'isomorphisme canonique $\mathrm{H}^{0}(\mathbf{P}(E), \mathcal{O}(\ell)) \stackrel{\sim}{\longrightarrow} S^{\ell}(E)$ induit une isométrie sur les $\mathbf{C}_{v}$-espaces vectoriels correspondants si $v$ est ultramétrique et une similitude de rapport $\left(\begin{array}{c}n-1+\ell \\ \ell\end{array}\right)^{1 / 2}$ si v est archimédienne.

Autrement dit, si $a_{n, \ell}$ désigne l'idèle $\left(\left(\begin{array}{c}n-1+\ell \\ \ell\end{array}\right)^{1 / 2}, \ldots,\left(\begin{array}{c}n-1+\ell \\ \ell\end{array}\right)^{1 / 2}\right.$, $1, \ldots, 1, \ldots)$ alors $S^{\ell}(\bar{E})$ s'identifie à $a_{n, \ell} \cdot \overline{E_{\ell}}$ (au sens de la page 45 , avec $a_{n, \ell}$ vu comme une homothétie de $E \otimes k_{\mathrm{A}}$ ). Ce lemme peut se démontrer en explicitant la norme $\|s(x)\|_{v, x, \ell}$ avec des coordonnées. Le choix d'une base $\left(X_{1}, \ldots, X_{n}\right)$ de $E_{1} \simeq E$ permet d'écrire $s$ comme un polynôme $P$ en les variables $X_{i}$ et si l'on désigne par $\left(x_{1}, \ldots, x_{n}\right) \in \mathbf{C}_{v}^{n}$ les coordonnées de $x$ dans cette base, on a la relation

$$
\|s(x)\|_{v, x, \ell}=\frac{\left|P\left(x_{1}, \ldots, x_{n}\right)\right|_{v}}{\|x\|_{E, v}^{\ell}} .
$$

On peut alors calculer les intégrales ci-dessus lorsque $v$ est archimédienne et si la norme $\|\cdot\|_{E, v}$ est hermitienne.

Proposition 7.7. Pour tout fibré vectoriel adélique $\bar{E}$ sur Spec $k$ et tout entier $\ell \geq 1$, la pente normalisée du fibré adélique hermitien $\overline{E_{\ell}}$ vérifie

$$
\left|\widehat{\mu}_{\mathrm{n}}\left(\overline{E_{\ell}}\right)-\ell \widehat{\mu}_{\mathrm{n}}(\bar{E})-\frac{\delta}{2} \log \left(\left(\begin{array}{c}
n-1+\ell \\
\ell
\end{array}\right) \gamma_{n, \ell}\right)\right| \leq \frac{\ell}{D} \log \Delta(\bar{E}) .
$$

Quant à la pente maximale de $\overline{E_{\ell}}$, la quantité

$$
\widehat{\mu}_{\max }\left(\overline{E_{\ell}}\right)-\ell \widehat{\mu}_{\max }(\bar{E})-\frac{\delta}{2} \log \left(\begin{array}{c}
n-1+\ell \\
\ell
\end{array}\right)
$$

est comprise entre $-\frac{\ell}{D} \log \Delta(\bar{E})$ et $\ell\left(2 \delta n \log n+\frac{1}{D} \log \Delta(\bar{E})\right)$.

DÉmonstration. Dans le cas hermitien, cette proposition est une simple conséquence du théorème $7.1 \mathrm{et} \mathrm{du} \mathrm{lemme} 7.3$ car $\overline{E_{\ell}}=a_{n, \ell}^{-1} \cdot S^{\ell}(\bar{E})$. Une fois ce cas établi, le cas général s'obtient en considérant le fibré $\bar{E}_{\varepsilon}$ (voir (28)). Il faut observer que les normes sur $E_{\ell}$ données par celles de $\overline{E_{\varepsilon}}$ et $\bar{E}$ vérifient des inégalités du même type que (28), à savoir que, pour tout 
sous espace vectoriel $E^{\prime}$ de $E_{\ell}$, on a

$$
\left(E^{\prime},\left(|\cdot|_{\left(E_{\varepsilon}\right)_{\ell}, v}\right)_{v}\right) \preceq\left(E^{\prime},\left(\|\cdot\|_{E_{\ell}, v}\right)_{v}\right) \preceq\left(E^{\prime},\left(\left(\mathrm{d}\left(E \otimes_{k} k_{v}, \ell_{n, k_{v}}^{2}\right)(1+\varepsilon)\right)^{\ell}|\cdot|_{\left(E_{\varepsilon}\right)_{\ell}, v}\right)_{v}\right) .
$$

Ce dernier énoncé offre une transition vers la géométrie algébrique et la géométrie diophantienne, domaines dans lesquels le formalisme des pentes trouve probablement sa raison d'être.

\section{Perspectives géométriques.}

Comme nous l'avons déjà mentionné dans l'introduction, le formalisme des pentes adéliques a des applications en géométrie diophantienne. Dans ce dernier paragraphe, nous souhaitons donner quelques pistes pour mieux comprendre l'usage que l'on peut faire de ces pentes dans un problème diophantien de nature géométrique. Dans cette optique, l'inégalité

$$
\widehat{\mu}_{\mathrm{n}}(\bar{E}) \leq \sum_{i=1}^{N} \frac{\operatorname{dim}\left(E_{i} / E_{i+1}\right)}{n}\left\{\widehat{\mu}_{\max }\left(\overline{G_{i}}\right)+h\left(\overline{E_{i}}, \overline{G_{i}} ; \varphi_{i}\right)\right\}+\frac{1}{D} \log \operatorname{vr}(\bar{E})
$$

de la proposition 6.6 joue un rôle important même si elle n'est finalement qu'une version savante de la formule du produit, ou de ce que l'on appelle en Transcendance l'inégalité de Liouville. Quoi qu'il en soit, elle est l'axe autour duquel s'articule la méthode des pentes, méthode conçue par J.B. Bost [6] et utilisée par exemple dans les articles [16, 18, 19, 39]. Dans un contexte géométrique, les objets $\bar{E}, \overline{G_{i}}, \varphi$ sont souvent choisis de la manière suivante. On considère une variété projective $X$ sur un corps global $k$ et $L \rightarrow X$ un fibré en droites ample sur $X$. On pose $E:=\mathrm{H}^{0}\left(X, L^{\otimes m}\right)$ l'espace vectoriel des sections globales d'une puissance entière $m$ de $L$. On se donne également un sous-schéma fermé $\Sigma$ de $X$, fini, composé de points épaissis de $X(k)$ dans certaines directions à divers ordres. Un point de $\Sigma$ procède d'un triplet constitué d'un point $k$-rationnel $x$ de $X$, d'une direction d'épaississement (p. ex. cela peut être un sous-espace vectoriel de l'espace tangent $t_{X, x}$ à $X$ au point $x$ ) et d'un ordre de dérivation. L'objectif de la méthode des pentes est de dégager quelques propriétés diophantiennes de $\Sigma$. Typiquement, on dispose d'un certain nombres de points complexes de $X$ dont on cherche à prouver la transcendance. On les suppose algébriques et on forme un schéma $\Sigma$ avec cette hypothèse. L'application linéaire $\varphi$ est le morphisme d'évaluation qui à une section $s \in E$ associe la restriction $s_{\mid \Sigma}$ de $s$ à $\Sigma$. La 
filtration $\left(F_{i}\right)_{i \in\{0, \ldots, N\}}$ de $F:=\mathrm{H}^{0}\left(\Sigma, L_{\mid \Sigma}^{\otimes m}\right)$ est choisie de manière à annuler successivement les points de $\Sigma$ et les ordres de dérivations correspondants. L'espace quotient $G_{i}$ s'identifie à un sous-espace de $S^{\ell}\left(t_{X, x}^{\vee}\right) \otimes x^{*} L^{\otimes m}$ où $\ell \in \mathbf{N}$ et $x \in \Sigma$. Afin de mettre en œuvre l'inégalité de pentes (54), il faut choisir des structures adéliques sur $E$ et les $G_{i}$, suffisamment appropriées pour que la pente normalisée $\widehat{\mu}_{\mathrm{n}}(\bar{E})$, la pente maximale $\widehat{\mu}_{\max }\left(\overline{t_{X, x}^{v}}\right)$ et le degré normalisé $\operatorname{deg}_{\mathrm{n}} x^{*} L$ puissent être évalués en fonction d'invariants attachés à $X, L, x$. Nous allons détailler cela dans un instant. Soulignons auparavant que, pour espérer obtenir quelques informations sur $\Sigma$, il importe en général d'avoir des données rendues dynamiques par l'introduction de paramètres tels que l'entier $m$ dans la définition de $E$ ou bien ceux qui sont dissimulés dans la définition de $\Sigma$ (ordres d'annulations en chaque point $x$ ).

Revenons maintenant sur le choix des métriques. Pour ce qui est de l'espace tangent $t_{X, x}$, on peut le munir d'une structure entière provenant d'un modèle $\left(X \rightarrow \operatorname{Spec} \mathcal{O}_{k}, \varepsilon_{x}: \operatorname{Spec} \mathcal{O}_{k} \rightarrow X\right)$ de $(X, x)$, lisse en $\varepsilon_{x}$, où $\mathcal{O}_{k}$ désigne l'anneau des entiers de $k$. Aux places archimédiennes $v$ de $k$, la première forme de Chern $c_{1}\left(L_{v}\right)$ du fibré ample $L_{v} \rightarrow X \times \operatorname{Spec} \mathbf{C}_{v}$, induit par $L$, fournit des métriques hermitiennes sur $t_{X, x} \otimes_{k} \mathbf{C}_{v}$.

En ce qui concerne les structures adéliques de $E$ et de $x^{*} L$, on peut distinguer deux écoles : soit l'on choisit avec soin les métriques pour en obtenir de canoniques et l'on calcule alors explicitement les quantités $\widehat{\mu}_{\mathrm{n}}(\bar{E})$ et $\widehat{\operatorname{deg}}_{\mathrm{n}} x^{*} L$ (cette dernière étant alors une hauteur canonique de $x$ ); soit, a contrario, on opte pour une très grande souplesse dans le choix des métriques, avec le minimum de contraintes, et l'on se contente de formules asymptotiques pour ces quantités avec $L$ remplacé par $L^{\otimes m}$ et $m \rightarrow+\infty$. D'une manière générale, la première option est celle qui prédomine en géométrie d'Arakelov. Mentionnons, à titre d'exemple, le cas d'une variété abélienne $X$ sur un corps de nombres $k$, munie d'un fibré en droites $L$ ample et symétrique. En s'appuyant sur les travaux de L. Moret-Bailly, J.-B. Bost a montré comment choisir un modèle dit cubiste de $(X, L, \Sigma)$ afin de calculer explicitement la pente normalisée de $\overline{\mathrm{H}^{0}(X, L)}$ en termes de la hauteur de Faltings $h_{F}(X)$ de $X$ et du degré géométrique $\operatorname{deg}_{L} X$; la formule exacte est

$$
\widehat{\mu}_{\mathrm{n}}\left(\overline{\mathrm{H}^{0}(X, L)}\right)=-\frac{1}{2} h_{F}(X)+\frac{1}{4} \log \frac{\operatorname{deg}_{L} X}{(2 \pi)^{d} d !} \quad\left(d:=\operatorname{dim}_{k} X\right) .
$$

Dans ce cas le degré normalisé $\widehat{\operatorname{deg}}_{\mathrm{n}} \overline{x^{*} L}$ est la hauteur de Néron-Tate de $x$ relative à $L$ (voir le théorème 5.10 de [5]). Toutefois les choix des métriques et les calculs qui en découlent sont assez délicats, utilisant des résultats 
profonds comme le théorème de Riemann-Roch arithmétique. Aussi lorsque le problème s'y prête - il est parfois préférable de se contenter de formules asymptotiques pour $\widehat{\mu}_{\mathrm{n}}\left(\overline{\mathrm{H}^{0}\left(X, L^{\otimes m}\right)}\right)$, lorsque $m \rightarrow+\infty$ (théorème de Hilbert-Samuel arithmético-géométrique). D'autant plus, peut-être, depuis la parution du mémoire de R. Rumely, C.F. Lau \& R. Varley [28] qui fournit un énoncé de ce type, sous des hypothèses très faibles. Pour mieux comprendre de quoi il s'agit, nous allons énoncer le résultat principal de [28] (théorème (A) p. 4) avec des hypothèses un peu plus fortes (p. ex. nous prenons des normes au lieu de semi-normes). Ce résultat donne une illustration de la manière avec laquelle on procède pour donner une structure adélique à $\mathrm{H}^{0}\left(X, L^{\otimes m}\right)$ et $x^{*} L$ et d'un calcul de pente normalisée non trivial. Étant donné une place $v$ de $k$ et $x \in X\left(\mathbf{C}_{v}\right)$, on considère une norme $\|\cdot\|_{L, v, x}$ sur la fibre $L_{x} \otimes_{k} \mathbf{C}_{v}$, invariante sous l'action de $\operatorname{Gal}\left(\mathbf{C}_{v} / k_{v}\right)$, norme que l'on transmet à $L_{x}^{\otimes m} \otimes_{k} \mathbf{C}_{v}$ par produit tensoriel. On suppose que, pour tout $x \in X(k)$, pour tout élément $e \in L_{x} \backslash\{0\}$, on a $\|e\|_{L, v, x}=1$ pour toute place $v$ en dehors d'un nombre fini. Pour $s \in \mathrm{H}^{0}\left(X, L^{\otimes m}\right) \otimes_{k} \mathbf{C}_{v}$, on pose alors $\|s\|_{v}:=\sup _{x \in X\left(\mathbf{C}_{v}\right)}\|s(x)\|_{L^{\otimes m}, v, x}$. Cela définit une structure adélique sur $\mathrm{H}^{0}\left(X, L^{\otimes m}\right)$ dès lors que $\|s\|_{v}$ est toujours fini et même un peu plus, à savoir que si $s \neq 0$ et pour toute place $v$ en dehors d'un ensemble fini (qui peut dépendre de $s$ ), on requiert $\|s\|_{v}=1$. Là encore, le choix des normes $\|\cdot\|_{L, v, x}$ aux places ultramétriques $v$ de $k$ peut se faire au moyen d'un modèle entier $(X, L) \operatorname{sur} \operatorname{Spec} \mathcal{O}_{k}$ de $(X, L)$ (voir op. cit.).

THÉORÈME [28]. Soit X une variété projective sur un corps global k. On suppose que $X$ est équidimensionnelle et géométriquement réduite. Soit $L \rightarrow X$ un fibré en droites ample, muni de métriques comme ci-dessus, conférant à $\mathrm{H}^{0}\left(X, L^{\otimes m}\right)$ une structure de fibré vectoriel adélique, pour tout entier $m \geq 1$. Alors il existe un élément $h_{\bar{L}}(X) \in \mathbf{R} \cup\{+\infty\}$ tel que

$$
\frac{1}{m} \widehat{\mu}_{\mathrm{n}}\left(\overline{\mathrm{H}^{0}\left(X, L^{\otimes m}\right)}\right) \underset{m \rightarrow+\infty}{\longrightarrow} h_{\bar{L}}(X),
$$

élément qui s'exprime en fonction de la capacité sectionnelle $S_{\gamma}(\bar{L})$ de $\bar{L}$ par la formule

$$
h_{\bar{L}}(X)=-\frac{\log S_{\gamma}(\bar{L})}{D(d+1) \operatorname{deg}_{L} X}
$$

$\left(d=\operatorname{dim}_{k} X, D=\left[k: k_{0}\right]\right)$.

Nous avons noté à dessein $h_{\bar{L}}(X)$ la limite ci-dessus car, lorsqu'elle est finie (et l'on connaît un critère pour que ce soit le cas, $i b i d$. ), les propriétés de la capacité sectionnelle font que cette quantité se comporte effective- 
ment comme une hauteur canonique de $X$ relativement à $\bar{L}$ (voir [11, 41] ainsi que le théorème (B) de [28]).

Lorsque $X$ est une variété abélienne sur un corps de nombres et $\bar{L}$ un fibré en droites cubiste, on a $h_{\bar{L}}(X)=0$. On peut observer que l'utilisation conjointe d'un résultat asymptotique tel que (55) et de l'inégalité de pentes (54) n'est pas entravée par la présence du terme d'erreur $\frac{1}{D} \log \operatorname{vr}\left(\overline{\mathrm{H}^{0}\left(X, L^{\otimes m}\right)}\right)$, logarithmique en $m$ et donc négligeable devant $m$.

\section{Annexe.}

Nous établissons l'estimation asymptotique suivante, mentionnée au paragraphe 7 .

Proposition. Soit $n, \ell \in \mathbf{N} \backslash\{0\}$ et $\gamma_{n, \ell}$ le nombre réel positif défini par la formule

$$
\log \gamma_{n, \ell}=\frac{1}{\left(\begin{array}{c}
\ell+n-1 \\
n-1
\end{array}\right)} \sum_{\substack{\mathbf{i} \in \mathbf{N} n \\
|\mathbf{i}|=\ell}} \log \frac{\ell !}{\mathbf{i} !} .
$$

Posons $H_{n}:=\sum_{i=1}^{n} 1 / i$. Alors, lorsque n est fixé et $\ell$ tend vers $+\infty$, on a

$$
\log \gamma_{n, \ell}=\left(H_{n}-1\right)(\ell+o(\ell)) .
$$

DÉmonstration. Le cas $n=1$ est immédiat et l'on suppose $n \geq 2$ pour cette démonstration. Notons $i_{1}, \ldots, i_{n}$ les composantes de $\mathbf{i} \in \mathbf{N}^{n}$. Soit $f:[0,+\infty[\rightarrow \mathbf{R}$ la fonction continue définie par $f(0)=0$ et, si $x>0$, $f(x)=-x \log x$. En vertu de la formule de Stirling, rappelée au bas de la page 29 , le terme $\log \frac{\ell !}{\mathrm{i} !} \mathrm{s}$ 'écrit $\sum_{h=1}^{n} f\left(i_{h} / \ell\right)+\mathrm{O}(\log \ell)$. On a alors

$$
\frac{\log \gamma_{n, \ell}}{\ell(n-1) !} \underset{\ell \rightarrow \infty}{\sim} \frac{1}{\ell^{n-1}} \sum_{|\mathbf{i}|=\ell} \sum_{h=1}^{n} f\left(\frac{i_{h}}{\ell}\right) \text {. }
$$

On reconnait pour le membre de droite la somme

$$
\frac{1}{\ell^{n-1}} \sum_{0 p \mathbf{j} \in \mathbf{N}^{n-1}|\mathbf{j}| \leq \ell}\left\{f\left(\frac{j_{1}}{\ell}\right)+\cdots+f\left(\frac{j_{n-1}}{\ell}\right)+f\left(1-\sum_{h=1}^{n-1} \frac{j_{h}}{\ell}\right)\right\},
$$

qui est une somme de Riemann $(n-1)$-dimensionnelle convergeant vers l'intégrale

$$
\int_{\Omega}\left(f\left(x_{1}\right)+\cdots+f\left(x_{n-1}\right)+f\left(1-x_{1}-\cdots-x_{n-1}\right)\right) \mathrm{d} x_{1} \ldots \mathrm{d} x_{n-1}
$$


sur l'ouvert $\Omega:=\left\{\left(x_{1}, \ldots, x_{n-1}\right) \in\right] 0,+\infty\left[^{n-1} ; x_{1}+\cdots+x_{n-1}<1\right\}$. Par symétrie, les intégrales $\int_{\Omega} f\left(x_{i}\right), 1 \leq i \leq n-1$, sont toutes égales. Par changement de variable $y:=1-x_{1}-\cdots-x_{n-1}$, qui ne change pas le domaine $\Omega$, la dernière intégrande de (56) peut être remplacée par $f\left(x_{n-1}\right)$, et l'intégrale (56) vaut alors

$$
\begin{aligned}
n \int_{\Omega} f\left(x_{n-1}\right) \mathrm{d} x_{1} \ldots \mathrm{d} x_{n-1} & =n \int_{0}^{1} f(u) \operatorname{vol}\left(x_{1}+\cdots+x_{n-2} \leq 1-u\right) \mathrm{d} u \\
& =\frac{n \operatorname{vol}\left(b_{n-2}^{1}\right)}{2^{n-2}} \int_{0}^{1}(1-u)^{n-2} f(u) \mathrm{d} u
\end{aligned}
$$

(ici vol est la mesure de Lebesgue sur $\mathbf{R}^{n-2}$ ). La formule (4) donne $n \operatorname{vol}\left(b_{n-2}^{1}\right) / 2^{n-2}=n /(n-2)$ !. Et la dernière intégrale se calcule au moyen de deux intégrations par partie (en observant que $\int_{0}^{1} \frac{1-u^{n}}{1-u} \mathrm{~d} u=H_{n}$ ). On trouve $\int_{0}^{1}(1-u)^{n-2} f(u) \mathrm{d} u=\frac{1}{n(n-1)}\left(H_{n}-1\right)$, ce qui permet de conclure.

Cette démonstration revêt un caractère ad hoc. Avec une analyse du terme reste de la somme de Riemann en fonction de la première dérivée de $f$, on peut montrer que $\log \gamma_{n, \ell}=\left(H_{n}-1\right) \ell+\mathrm{O}(\log \ell)$. Une vision plus savante et inspirée de ce genre de calculs se trouve dans la thèse de H. Randriambololona, dans laquelle le lecteur pourra trouver une évaluation asymptotique à un ordre quelconque de (variantes de) $\log \gamma_{n, \ell}$ et d'autres quantités d'origine géométrique, plus générales (voir [24], chapitre 4 , proposition 4.2.3 et supra).

\section{REFERENCES}

[1] W. Blaschke, Über affine Geometrie VII: Neue Extremeigenschaften von Ellipse und Ellipsoid. Ber. Vehr. Sächs. Akad. Wiss. Leipzig Math.-Phys. Kl, 69 (1917), pp. 306-318.

[2] E. Bombieri - J. VAaler, On Siegel's lemma. Invent. Math., 73 (1) (1983), pp. 11-32, Avec un addendum : ibid. 75(2) (1984), p. 377.

[3] T. BoREK, Successive minima and slopes of hermitian vector bundles over number fields. J. Number Theory, 113 (2) (2005), pp. 380-388.

[4] J.-B. Bost - H. Gillet - C. Soulé, Heights of projective varieties and positive Green forms. J. Amer. Math. Soc., 7 (4) (1994), pp. 903-1027.

[5] J.-B. Bost, Intrinsic heights of stable varieties and abelian varieties. Duke Math. J., 82 (1) (1996), pp. 21-70.

[6] J.-B. Bost, Périodes et isogénies des variétés abéliennes sur les corps de nombres (d'après D. Masser et G. Wüstholz). Séminaire Bourbaki, Exp. 
no 795. Volume 237 d'Astérisque, pp. 115-161. Société Mathématique de France, 1996.

[7] J.-B. Bost, Algebraic leaves of algebraic foliations over number fields. Publ. Math. Inst. Hautes Études Sci, 93 (2001), pp. 161-221.

[8] N. Bourbaki, Éléments de mathématiques. Fasc. XXX. Algèbre commutative. Chapitre 5 : Entiers. Chapitre 6 : Valuations. Actualités Scientifiques et Industrielles, No. 1308. Hermann (Paris), 1964.

[9] N. BourbaKI, Espaces vectoriels topologiques. Chapitres 1 à 5 . Éléments de mathématique. Nouvelle édition. Masson, Paris, 1981.

[10] J. Bourgain - V. Milman, New volume ratio properties for convex symmetric bodies in $\mathbf{R}^{n}$. Invent. Math., 88 (2) (1987) pp. 319-340.

[11] A. Chambert-Loir, Points de petite hauteur sur les variétés semi-abéliennes. Ann. Sci. École Norm. Sup., 33 (6) (2000), pp. 789-821.

[12] A. Chambert-Loir, Théorèmes d'algébricité en géométrie diophantienne (d'après J.-B. Bost, Y. André, D. \& G. Chudnovsky). Séminaire Bourbaki, Exp. no 886. Volume 282 d'Astérisque, 175-209. Société Mathématique de France, 2002.

[13] C. Chevalley, Introduction to the theory of algebraic functions of one variable. Mathematical surveys, no. VI, American Mathematical Society, 1951.

[14] A. Defant - K. Floret, Tensor norms and operator ideals, volume 176 de North-Holland Mathematics Studies, North-Holland Publishing Co., Amsterdam, 1993.

[15] K. FLORET, The extension theorem for norms on symmetric tensor products of normed spaces. Recent progress in functional analysis (Valencia, 2000), volume 189 de North-Holland Math. Stud., 225-237. North-Holland, 2001.

[16] É. GaUdron, Formes linéaires de logarithmes effectives sur les variétés abéliennes. Ann. Sci. École Norm. Sup., 39 (5) (2006), pp. 699-773.

[17] É. Gaudron, Étude du cas rationnel de la théorie des formes linéaires de logarithmes. J. Number Theory, 127 (2) (2007), pp. 220-261.

[18] P. Graftieaux, Formal groups and the isogeny theorem. Duke Math. J., 106 (1) (2001) pp. 81-121.

[19] P. Graftieaux, Formal subgroups of abelian varieties. Invent. Math., 145 (1) (2001), pp. 1-17.

[20] S. LANG, Algebraic number theory, volume 110 de Graduate Texts in Mathematics, Springer-Verlag, N. Y., 1994.

[21] K. MAHLER, Ein Übertragungsprinzip für konvexe Körper. Casopis Pest. Mat. Fys., 68 (1939), pp. 93-102,.

[22] V. MaIllot, Géométrie d'Arakelov des variétés toriques et fibrés en droites intégrables, volume 80 de Mémoire de la société mathématique de France, S. M. F., 2000.

[23] G. PISIER, The volume of convex bodies and Banach space geometry, volume 94 de Cambridge Tracts in Mathematics, Cambridge University press, 1989.

[24] H. RandRIAmbololona, Hauteurs pour les sous-schémas et exemples d'utilisation de méthodes arakeloviennes en théorie de l'approximation diophantienne, Thèse de doctorat, Université Paris XI (Orsay), janvier 2002.

[25] R. Remmert, Classical topics in complex function theory, volume 172 de Graduate Texts in Mathematics, Springer-Verlag, N. Y., 1998. 
[26] M. RogaLski, Sur le quotient volumique d'un espace de dimension finie, Séminaire Initiation à l'Analyse, G. Choquet, M. Rogalski, J. Saint-Raymond, 20e année, 1980/81, no. 3. Volume 46 de Publ. Math. univ. Pierre et Marie Curie, Paris VI.

[27] D. Roy - J.L. Thunder, An absolute Siegel's lemma. J. Reine angew. Math., 476 (1996), pp. 1-26. Addendum et erratum, ibid., 508 (1999), 47-51.

[28] R. Rumely - C.F. LAU - R. VARLey, Existence of the sectional capacity, volume 145, n. 690, de Memoirs of the American Mathematical Society, A.M.S., 2000.

[29] R.A. RYAN, Introduction to tensor products of Banach spaces. Springer Monographs in Math. Springer-Verlag London Ltd., 2002.

[30] J. SAInT-RAYmond, Sur le volume des corps convexes symétriques, Séminaire Initiation à l'Analyse, G. Choquet, M. Rogalski, J. Saint-Raymond, 20e année, 1980/81, no. 11. Volume 46 de Publ. Math. univ. Pierre et Marie Curie, Paris VI.

[31] L.A. SANTALó, Un invariante afin para los cuerpos convexos del espacio de $n$ dimensiones. Portugal Math., 8 (1949), pp. 155-161.

[32] W.M. Schmidt, A remark on the heights of subspaces. A tribute to Paul Erdös, pp. 359-360. Cambridge Univ. Press, 1990.

[33] R. Schneider, Convex bodies: the Brunn-Minkowski theory, volume 44 de Enyclopedia of mathematics and its applications, Cambridge University Press, 1993.

[34] C. SoulÉ, Successive minima on arithmetic varieties. Compositio Math., 96 (1) (1995), pp. 85-98.

[35] T. STRUPPECK - J.D. VAALER, Inequalities for heights of algebraic subspaces and the Thue-Siegel principle. Analytic number theory (Allerton Park, IL, 1989), volume 85 de Progress in Math., 493-528. Birkhäuser Boston, 1990.

[36] L. SzPIRo, Degrés, intersections, hauteurs. Séminaire sur les pinceaux arithmétiques : la conjecture de Mordell, volume 127 d'Astérique, 11-28. Société Mathématique de France, 1985.

[37] A.C. Thompson, Minkowski geometry, volume 63 de Encyclopedia of Mathematics and its Applications, Cambridge University Press, 1996.

[38] J.L. Thunder, An adelic Minkowski-Hlawka theorem and an application to Siegel's lemma. J. reine angew Math., 475 (1996), pp. 167-185.

[39] E. VIADA, Slopes and abelian subvariety theorem. J. Number Theory, 112 (1) (2005) pp. 67-115.

[40] A. WEIL, Basic number theory, Seconde édition de 1973 publiée dans Classics in Mathematics, Springer-Verlag, Berlin, 1995.

[41] S. Zhang, Positive line bundles on arithmetic varieties. J. Amer. Math. Soc., 8 (1) (1995), pp. 187-221.

Manoscritto pervenuto in redazione il 30 maggio 2006. 
